\title{
NEXT ORDER ASYMPTOTICS AND RENORMALIZED ENERGY FOR RIESZ INTERACTIONS
}

\author{
MIRCEA PETRACHE ${ }^{1}$ AND SYLVIA SERFATY ${ }^{1,2}$ \\ ${ }^{1}$ UPMC Univ. Paris 6, UMR 7598 Laboratoire Jacques-Louis Lions, \\ Paris, F-75005, France (mircea.petrache@upmc.fr) \\ ${ }^{2}$ Courant Institute, New York University, 251 Mercer st, New York, \\ NY 10012, USA (serfaty@ann.jussieu.fr)
}

(Received 30 September 2014; accepted 5 May 2015; first published online 29 May 2015)

Abstract We study systems of $n$ points in the Euclidean space of dimension $d \geqslant 1$ interacting via a Riesz kernel $|x|^{-s}$ and confined by an external potential, in the regime where $d-2 \leqslant s<d$. We also treat the case of logarithmic interactions in dimensions 1 and 2. Our study includes and retrieves all cases previously studied in Sandier and Serfaty [2D Coulomb gases and the renormalized energy, Ann. Probab. (to appear); 1D log gases and the renormalized energy: crystallization at vanishing temperature (2013)] and Rougerie and Serfaty [Higher dimensional Coulomb gases and renormalized energy functionals, Comm. Pure Appl. Math. (to appear)]. Our approach is based on the Caffarelli-Silvestre extension formula, which allows one to view the Riesz kernel as the kernel of an (inhomogeneous) local operator in the extended space $\mathbb{R}^{d+1}$.

As $n \rightarrow \infty$, we exhibit a next to leading order term in $n^{1+s / d}$ in the asymptotic expansion of the total energy of the system, where the constant term in factor of $n^{1+s / d}$ depends on the microscopic arrangement of the points and is expressed in terms of a 'renormalized energy'. This new object is expected to penalize the disorder of an infinite set of points in whole space, and to be minimized by Bravais lattice (or crystalline) configurations. We give applications to the statistical mechanics in the case where temperature is added to the system, and identify an expected 'crystallization regime'. We also obtain a result of separation of the points for minimizers of the energy.

Keywords: Riesz energy; renormalized energy; Fekete points; point separation

2010 Mathematics subject classification: 82B05; 82B21; 82B26; $15 \mathrm{~B} 52$

\section{Introduction}

We study the equilibrium properties of a system of $n$ points in the full space of dimension $d \geqslant 1$, interacting via Riesz kernel interactions and confined by an 'external field' or potential $V$. More precisely, we are considering energies (or Hamiltonians) of the form

$$
H_{n}\left(x_{1}, \ldots, x_{n}\right)=\sum_{i \neq j} g\left(x_{i}-x_{j}\right)+n \sum_{i=1}^{n} V\left(x_{i}\right)
$$


where $x_{1}, \ldots, x_{n}$ are $n$ points in $\mathbb{R}^{d}$ and the interaction kernel is given by either

$$
g(x)=\frac{1}{|x|^{s}} \quad \max (0, d-2) \leqslant s<d,
$$

or

$$
g(x)=-\log |x| \quad \text { in dimension } d=1,
$$

or

$$
g(x)=-\log |x| \quad \text { in dimension } d=2 .
$$

We are interested in the asymptotics $n \rightarrow \infty$ of the minimum of $H_{n}$. Note here that the factor $n$ in front of the second term in (1.1) puts us in a mean-field scaling where the potential term and the pair-interaction terms are of the same order of magnitude. This choice is equivalent to demanding that the pair-interaction strength be of order $n^{-1}$. One can always reduce to this situation in the particular case where the confining potential $V$ has some homogeneity.

The case when $s=d-2$ for $d \geqslant 3$ and case (1.4) correspond to the Coulomb interaction cases in dimension $d \geqslant 2$. The cases when $d-2<s<d$ correspond to more general Riesz interactions. Systems of points with Riesz interaction have particularly attracted attention in approximation theory. We refer to the forthcoming monograph of Borodachov, Hardin, and Saff [7] and the review papers [12, 45] and references therein. Such systems are mostly studied on the $d$-dimensional sphere or torus, but studying them in Euclidean space with an external confining potential $V$ is also of interest, as it can also correspond to physically meaningful particle systems, or can provide tools to study such interactions on manifolds. The Coulomb case was already studied in [44, 48], while case (1.3) was studied in [49]. We refer to these papers and the book of Forrester [29] for references on these classical cases of Coulomb and log gases and their importance in mathematical physics and random matrix theory. Here we generalize these approaches, in particular that of [44], to the case of non-Coulomb interaction, and we retrieve by the same token most of the results obtained in [44, 48, 49].

In approximation theory (see references above) the whole range $s \in \mathbb{R}$ is of interest. The case when $s \geqslant d$ is called the hypersingular case and the case when $s<d$ is called the the potential case (cf. [33]). When $s \rightarrow \infty$ the problem connects with best packing problems, and when $s \rightarrow 0$ it connects by means of $\left(|\cdot|^{-s}-1\right) / s \rightarrow-\log |\cdot|$ to the logarithmic interaction $-\sum_{i \neq j} \log \left|x_{i}-x_{j}\right|$, whose minimization is equivalent to the maximization of the product of distances $\prod_{i \neq j}\left|x_{i}-x_{j}\right|$, i.e. to Fekete points. These are related to orthogonal polynomials and are of major interest in interpolation theory. Cases (1.3)-(1.4) which we study here correspond exactly to one-dimensional and two-dimensional weighted Fekete points (cf. [46]) or physically to the 'log gas' systems mentioned above (cf. [29]).

It is well known since [20] that to leading order

$$
\min H_{n}=n^{2} \mathcal{E}\left(\mu_{V}\right)+o\left(n^{2}\right)
$$

in the limit $n \rightarrow \infty$, where

$$
\mathcal{E}(\mu)=\iint_{\mathbb{R}^{d} \times \mathbb{R}^{d}} g(x-y) d \mu(x) d \mu(y)+\int_{\mathbb{R}^{d}} V(x) d \mu(x)
$$


is the mean-field energy functional defined for Radon measures $\mu$, and the equilibrium measure $\mu_{V}$ is the minimizer of $\mathcal{E}$ in the space of probability measures on $\mathbb{R}^{d}$, denoted $\mathcal{P}\left(\mathbb{R}^{d}\right)$. This is true only for $s<d$, which is the condition for (1.6) to make sense and to have a minimizer. This is why this case is called the potential case.

In this paper we characterize the next to leading order term, by showing among other things the following sample result (for more details, see Theorem 4).

Theorem 1. Assume (1.2) and that $V$ is such that the equilibrium measure $\mu_{V}$ exists and satisfies some suitable regularity assumptions (in particular has a density; see below). Then we have the expansion

$$
\min H_{n}=n^{2} \mathcal{E}\left(\mu_{V}\right)+n^{1+\frac{s}{d}} C_{d, s, V}+o\left(n^{1+\frac{s}{d}}\right)
$$

where

$$
C_{d, s, V}=\xi_{d, s} \int_{\mathbb{R}^{d}} \mu_{V}^{1+\frac{s}{d}}(x) d x
$$

and the number $\xi_{d, s}$ depends only on $d$ and $s$ and is characterized as the minimum of a function $\mathcal{W}$ described below.

The fact that the next order term lies at order $n^{1+s / d}$ is not difficult to guess by scaling, but the existence of an asymptotic limit for it was the main open question. In the context of the Riesz energy on the sphere, this is listed as a conjecture (with a precise conjectured value for the constant if $d=2,4,8,24)$ in [12, Conjecture 3]. In addition to finding the existence of the asymptotic term, we exhibit a new object, called 'renormalized energy' and denoted $\mathcal{W}$, which governs this order in the energy for arbitrary (possibly nonminimizing) configurations.

Our method relies on expressing the interaction as a quadratic integral of the potential generated by the point configuration via

$$
g * \sum_{i} \delta_{x_{i}}
$$

and expanding this integral interaction to next order in $n$ to obtain a next order asymptotic limit of the energy, by an exact splitting formula, as done in [44, 48, 49]. The main difference is that here the Riesz kernel $g$ is not the convolution kernel of a local operator, as in the Coulomb case $s=d-2$ or (1.4), where $g$ is the kernel of the (inverse) Laplacian operator. Instead it is the kernel of a nonlocal one, more precisely a fractional Laplacian. It turns out however that, if $d-2<s<d$, this fractional Laplacian nonlocal operator can be transformed into a local but inhomogeneous operator of the form $\operatorname{div}\left(|y|^{\gamma} \nabla \cdot\right)$ by adding one space variable $y \in \mathbb{R}$ to the space $\mathbb{R}^{d}$. In the particular case of $s=d-1$ then $\gamma=0$, and this corresponds to using a harmonic extension, a relatively common procedure which seems to have originated in the probability literature in [38]. In the more general setting, the extension procedure is due to Caffarelli and Silvestre [13], and has been much used to study nonlocal partial differential equations involving fractional Laplacians. Using it in our context is in line with [49], where the harmonic extension from dimension 1 to 2 was used to transform the one-dimensional logarithmic interaction into the two-dimensional Coulomb interaction. We note that the 
boundaries of the constraint $s \in[d-2, d)$ we inherit from this approach are quite natural: $d-2$ is the Coulomb case, and $s<d$ is the regime for which a potential theory associated to the Riesz kernel exists [33]. The constraint $s \in[d-2, d)$ also appears in most of the results on the sphere in $[10,11]$.

Transforming the nonlocal relation into a local one (in an extended space) allows us to follow the strategy of $[44,48]$; however, work is required to show that the strategy still works in an extended space and with an inhomogeneous operator. Also, [44] relied on Onsager's lemma, which itself relies on Newton's theorem (that any point charge generates outside of a ball the same Coulomb potential as the same charge which has been radially smeared out in the ball), only valid for Coulomb potentials. Here, we replace that use with a simple truncation procedure (a simplification which works in the Coulomb case too).

The proof of our main result relies first on proving lower bounds for the next order in $H_{n}$ of a generic configuration in terms of a limiting energy $\widetilde{\mathcal{W}}$ that we introduce, and second in constructing test configurations which allow us to achieve the value $\min \tilde{\mathcal{W}}$. Just as in [44, 48], the upper bound construction relies on a 'screening procedure' of a generic configuration. The proof of this screening takes up a large part of the paper, since it needs to be completely redone in the extended space $\mathbb{R}^{d+1}$ with the corresponding inhomogeneous operator.

As a byproduct of these matching lower and upper bounds, we not only obtain the asymptotic expansion (1.7) but we also obtain information on the minimizers themselves: after a suitable rescaling, they have to minimize $\tilde{\mathcal{W}} . \tilde{\mathcal{W}}$ is an appropriate average of a quantity $\mathcal{W}$, which itself is the energy of an infinite configuration of discrete points in the whole space $\mathbb{R}^{d}$, with a suitable uniform 'neutralizing background' corresponding to the average point density. It is the Riesz analog of the renormalized energy that was introduced in the 2D Coulomb case first by Sandier and Serfaty [47, 48], and then in higher dimension by Rougerie and Serfaty [44].

Our analysis thus leads to the question of minimizing $\mathcal{W}$ itself. While we know how to prove the existence of minimizers of $\mathcal{W}$ and a few of their qualitative properties, the identification of its minimum remains widely open. The few exceptions are the case of the one-dimensional logarithmic interaction (this could very likely be extended to Riesz interactions), for which the minimum is proven in [49] to be achieved at the perfect lattice configuration $\mathbb{Z}$ (for a uniqueness result, see [34]), and the two-dimensional Coulomb case in [47], where it is shown that, within the class of configurations of points that are forming a perfect lattice, the minimizer is the 'Abrikosov' triangular lattice (with $\pi / 3$ angles). This relies heavily on the corresponding result from number theory [16] which asserts that in dimension 2 the minimum of the Epstein zeta function of a lattice of fixed volume is uniquely achieved by the triangular lattice. ${ }^{1}$ These results led to conjecturing in [47] that the triangular lattice indeed achieves the minimum of $\mathcal{W}$ among all possible configurations. By mapping the plane to the 2-sphere, Bétermin [5] showed

\footnotetext{
${ }^{1}$ Some analogous special lattices exist in dimensions 4,8 and 24 : the lattice $D_{4}$ in dimension $4, E_{8}$ in dimension 8, and the Leech lattice in dimension 24. They are proven in [50] to be local minimizers of the Epstein zeta function among lattices of fixed volume.
} 
that conjecture is equivalent to the conjecture of [12], itself formulated on the sphere. Here we prove the analogous result to that of $[26,47]$ in the more general Riesz case, i.e. that the triangular lattice is the minimizer of $\mathcal{W}$ among lattices of volume 1 . We may then naturally extend the previous conjecture to one that says that in dimension 2 the minimum of $\mathcal{W}$ for all $0 \leqslant s<2$ is achieved by the triangular lattice. For general dimension, we may also conjecture that the minimum of $\mathcal{W}$ for all $\max (0, d-2) \leqslant s<d$ is always achieved by some lattice, which in dimensions 4, 8, 24 is the lattice $D_{4}, E_{8}$, and Leech respectively; this can be expected to also be equivalent to Conjecture 3 in [12] in the corresponding cases. Note that the fact that these special lattices should be minimizing for a broad class of interaction kernels appears for example in the Cohn-Kumar conjecture [22, 23]. Finally, let us mention that a few such crystallization results are known mostly in one dimension, e.g. one-dimensional Coulomb gases (i.e. with the one-dimensional Coulomb interaction kernel $|x|$ which is not treated here) $[9,35,36]$, zeros of orthogonal polynomials [2], and in dimension 2 and 3 for some very particular interaction kernels [26, 28, 41, 51].

As mentioned, the approach used here allows us to retrieve all previous results $[44,48,49]$ in one unified approach, but also brings a few simplifications.

- We use a simple definition of the renormalized energy by truncation. This allows us to bypass the use of Onsager's lemma and Newton's theorem in [44]. As in [44], this also avoids the use of mass displacement of $[48,49]$ since the energy thus defined is immediately bounded below.

- By proving a monotonicity in the truncation parameter, we easily deduce that $\mathcal{W}$ is bounded from below without having to first prove that minimizing configurations have well-separated points (which we do prove later).

- Our proof of screening, which has many features in common with that of [49] and with that of [44], is also more general than both of them in that we screen (almost) arbitrary configurations and not only those that have well-separated points, a result of independent interest.

- The assumptions on the equilibrium measure are weakened, and we distinguish more precisely those that suffice for the lower bound and those that are needed for the upper bound.

In addition to our main results about minimizers, we obtain two additional results, which are stated at the end of the introduction. One is an independent result of good separation of points for energy minimizers. The other is an application of our method to the statistical mechanics model of particles interacting via $H_{n}$ with temperature, in particular a next order expansion of the partition function.

Let us now get into the specifics.

\subsection{The equilibrium measure and our assumptions}

We first place assumptions on $V$ that ensure the existence of the equilibrium $\mu_{V}$ from standard potential theory. 
$V$ is l.s.c. (lower semicontinuous) and bounded below

$\{x: V(x)<\infty\}$ has positive $g$-capacity

$\lim _{|x| \rightarrow \infty} V(x)=+\infty$ in cases $(1.2)$, respectively

$\lim _{|x| \rightarrow \infty} \frac{V(x)}{2}-\log |x|=+\infty$ in cases $(1.3)-(1.4)$.

The following theorem, due to Frostman (cf. also [46]), then gives the existence and characterization of the equilibrium measure.

Theorem 2 [30]. Assume that $V$ satisfies (1.8)-(1.10); then there exists a unique minimizer $\mu_{V} \in \mathcal{P}\left(\mathbb{R}^{d}\right)$ of $\mathcal{E}$, and $\mathcal{E}\left(\mu_{V}\right)$ is finite. Moreover, the following properties hold.

- $\Sigma:=\operatorname{Supp}\left(\mu_{V}\right)$ is bounded and has positive $g$-capacity.

- For $c:=\mathcal{E}\left(\mu_{V}\right)-\int \frac{V}{2} d \mu_{V}$ and $h^{\mu_{V}}(x):=\int g(x-y) d \mu_{V}(y)$, it holds that

$$
\begin{cases}h^{\mu_{V}}+\frac{V}{2} \geqslant c & \text { q.e. (quasi everywhere) } \\ h^{\mu_{V}}+\frac{V}{2}=c & \text { q.e. on } \Sigma .\end{cases}
$$

We will write

$$
\zeta:=h^{\mu_{V}}+\frac{V}{2}-c \geqslant 0 .
$$

We will assume that $\mu_{V}$ is really a $d$-dimensional measure (i.e. $\Sigma$ is a nice $d$-dimensional set), with a density, and, just as in $[44,48]$, in order to make the explicit constructions easier, we need to assume that this density (that we still denote $\mu_{V}$ ) is bounded and sufficiently regular on its support. More precisely, we make the following assumptions (which are technical and could certainly be somewhat relaxed):

$$
\partial \Sigma \text { is } C^{1}
$$

$\mu_{V}$ has a density which is $C^{0, \beta}$ in $\Sigma$,

$\exists c_{1}, c_{2}, \bar{m}>0$ s.t. $c_{1} \operatorname{dist}(x, \partial \Sigma)^{\alpha} \leqslant \mu_{V}(x) \leqslant \min \left(c_{2} \operatorname{dist}(x, \partial \Sigma)^{\alpha}, \bar{m}\right)<\infty$ in $\Sigma$, with the conditions

$$
0<\beta \leqslant 1, \quad 0 \leqslant \alpha \leqslant \frac{2 \beta d}{2 d-s} .
$$

Of course if $\alpha<1$ one should take $\beta=\alpha$, and if $\alpha \geqslant 1$ one should take $\beta=1$ and $\alpha \leqslant \frac{2 d}{d-s}$. These assumptions are meant to include the case of the semi-circle law $\frac{1}{2 \pi} \sqrt{4-x^{2}} \mathbb{1}_{|x|<2}$ arising for the quadratic potential in the setting (1.3). We also know that, in the Coulomb cases, a quadratic potential gives rise to an equilibrium measure which is a multiple of a characteristic function of a ball, also covered by our assumptions with $\alpha=0$. Finally, in the Riesz case, it was noticed in [19, Corollary 1.4] that any compactly supported radial profile can be obtained as the equilibrium measure associated to some potential. Our assumptions are thus never empty.

We also note that the problem of minimizing $\mathcal{E}$ can be recast as a fractional obstacle problem $[14,15,52]$; thus the regularity of $h^{\mu_{V}}$ and the free-boundary $\partial \Sigma$ as a function of $V$ are known. 


\subsection{The extension representation for the fractional Laplacian}

In what follows, $k$ will denote the dimension extension. We will take $k=0$ in all the Coulomb cases, i.e. $s=d-2$ and $d \geqslant 3$, or (1.4). In all other cases, we will need to take $k=1$. Points in the space $\mathbb{R}^{d}$ will be denoted by $x$, and points in the extended space $\mathbb{R}^{d+k}$ by $X$, with $X=(x, y), x \in \mathbb{R}^{d}, y \in \mathbb{R}^{k}$. We will often identify $\mathbb{R}^{d} \times\{0\}$ and $\mathbb{R}^{d}$. By balls $B_{R}$ or $B(X, R)$ we will mean balls in the space $\mathbb{R}^{d+k}$, unless otherwise specified.

The extension representation of [14] relies on the remark that, if $d-2+k+\gamma>1$, a function of the form $C /|x|^{d-2+k+\gamma}$ appears as the $y=0$ restriction of the fundamental solution

$$
G_{\gamma}(x, y)=\frac{C_{\gamma+d+k}}{\left(|x|^{2}+|y|^{2}\right)^{\frac{d-2+k+\gamma}{2}}}
$$

of the operator $\operatorname{div}\left(|y|^{\gamma} \nabla u\right)$ on $\mathbb{R}^{d} \times \mathbb{R}^{k}$. Here $C_{q}=\pi^{q / 2} \Gamma(q / 2-1) / 4$. What we mean by a fundamental solution is that it solves

$$
\begin{cases}\operatorname{div}\left(|y|^{\gamma} \nabla u\right)=0 & \text { on } \mathbb{R}^{d} \times\left(\mathbb{R}^{k} \backslash\{0\}\right), \\ -\lim _{|y| \downarrow 0}|y|^{\gamma+k-1} \partial_{y} u(\cdot, y)=\delta_{0} & \text { on } \mathbb{R}^{d} \times\{0\}\end{cases}
$$

where $\delta_{0}$ denotes the Dirac mass at the origin. It follows that, if $\gamma$ is chosen such that

$$
d-2+k+\gamma=s
$$

then, given a measure $\mu$ on $\mathbb{R}^{d}$, the potential $h^{\mu}(x)$ generated by $\mu$ defined in $\mathbb{R}^{d}$ by

$$
h^{\mu}(x)=g * \mu(x)=\int_{\mathbb{R}^{d}} \frac{1}{\left|x-x^{\prime}\right|^{s}} d \mu\left(x^{\prime}\right)
$$

can be extended to a function $h^{\mu}(X)$ on $\mathbb{R}^{d+k}$ defined by

$$
h^{\mu}(X)=\int_{\mathbb{R}^{d}} \frac{1}{\left|X-\left(x^{\prime}, 0\right)\right|^{s}} d \mu\left(x^{\prime}\right),
$$

and this function satisfies

$$
-\operatorname{div}\left(|y|^{\gamma} \nabla h^{\mu}\right)=c_{d, s} \mu \delta_{\mathbb{R}^{d}}
$$

where by $\delta_{\mathbb{R}^{d}}$ we mean the uniform measure on $\mathbb{R}^{d} \times\{0\}$, i.e. $\mu \delta_{\mathbb{R}^{d}}$ acts on test functions $\varphi$ by

$$
\int_{\mathbb{R}^{d+k}} \varphi(X) d\left(\mu \delta_{\mathbb{R}^{d}}\right)(X)=\int_{\mathbb{R}^{d}} \varphi(x, 0) d \mu(x),
$$

and

$$
c_{d, s}= \begin{cases}2 s \frac{2 \pi^{\frac{d}{2}} \Gamma\left(\frac{s+2-d}{2}\right)}{\Gamma\left(\frac{s+2}{2}\right)} & \text { for } s>\max (0, d-2), \\ (d-2) \frac{2 \pi^{\frac{d}{2}}}{\Gamma(d / 2)} & \text { for } s=d-2>0, \\ 2 \pi & \text { in cases }(1.3),(1.4) .\end{cases}
$$


In particular, $g(X)=|X|^{-s}$ seen as a function of $\mathbb{R}^{d+k}$ satisfies

$$
-\operatorname{div}\left(|y|^{\gamma} \nabla g\right)=c_{d, s} \delta_{0}
$$

In order to recover the Coulomb cases, it suffices to take $k=\gamma=0$. If $s>d-2$ we take $k=1$ and $\gamma$ satisfying (1.16). In case (1.3), we note that $g(x)=-\log |x|$ appears as the $y=0$ restriction of $-\log |X|$, which is (up to a factor $2 \pi$ ) the fundamental to the Laplacian operator in dimension $d+k=2$. In this case, we may thus choose $k=1$ and $\gamma=0, c_{d, s}=c_{1,0}=2 \pi$, and the potential $h^{\mu}=g * \mu$ still satisfies (1.19), while $g$ still satisfies (1.21). This is the procedure that was used in [49], and we see that this case naturally embeds into the Riesz setting we are studying.

To summarize, we will take

$$
\begin{aligned}
\text { in the case } \max (0, d-2)<s<d, & \text { then } k & =1, \gamma=s-d+2-k, \\
\text { in the case }(1.3), & \text { then } k & =1, \gamma=0,
\end{aligned}
$$

in the case (1.4) or $d \geqslant 3, s=d-2$, then $k=0, \gamma=0$.

We note that formula (1.16) always remains formally true when taking the convention that $s=0$ in the case when $g(x)=-\log |x|$, and we also note that the assumption $d-2 \leqslant$ $s<d$ implies that in all cases $\gamma \in(-1,1)$.

Through this extension procedure, we will be led to studying equations of the form (1.19). These are degenerate elliptic equations; however, they are associated to weights which are in the Muckenhoupt class $A_{2}$ for which there is a good elliptic theory [27].

\subsection{Definition of $\mathcal{W}$}

Before defining our renormalized energy $\mathcal{W}$, we define the truncated Riesz (or logarithmic) kernel as follows: for $1>\eta>0$ and $X \in \mathbb{R}^{d+k}$, let

$$
f_{\eta}(X)=(g(X)-g(\eta))_{+} .
$$

We note that the function $f_{\eta}$ vanishes outside of $B(0, \eta)$ and satisfies that

$$
\delta_{0}^{(\eta)}:=\frac{1}{c_{d, s}} \operatorname{div}\left(|y|^{\gamma} \nabla f_{\eta}\right)+\delta_{0}
$$

is a positive measure supported on $\partial B(0, \eta)$, and which is such that, for any test function $\varphi$,

$$
\int \varphi \delta_{0}^{(\eta)}=\frac{1}{c_{d, s}} \int_{\partial B(0, \eta)} \varphi(X)|y|^{\gamma} g^{\prime}(\eta) .
$$

(Here and later, since $g$ is radial, we abuse notation by writing $g^{\prime}$ for the derivative of $g$ seen as a function on $\mathbb{R}$.) One can thus check that $\delta_{0}^{(\eta)}$ is a positive measure of mass 1 , and we may write

$$
-\operatorname{div}\left(|y|^{\gamma} \nabla f_{\eta}\right)=c_{d, s}\left(\delta_{0}-\delta_{0}^{(\eta)}\right) \quad \text { in } \mathbb{R}^{d+k} .
$$

We will also denote by $\delta_{p}^{(\eta)}$ the measure $\delta_{0}^{(\eta)}(X-p)$, for $p \in \mathbb{R}^{d} \times\{0\}$. Again, we note that this includes cases $(1.23)-(1.24)$. In the Coulomb cases, i.e. when $k=0$, then $\delta_{0}^{(\eta)}$ is simply the normalized surface measure on $\partial B(0, \eta)$; it is thus a particular case of the 
radially symmetric smearing out performed in [44], and thus the renormalized energy we will define next is the same as in [44].

The renormalized energy of an infinite configuration of points is defined via the gradient of the potential generated by the point configuration, embedded into the extended space $\mathbb{R}^{d+k}$. That gradient is a vector field that we denote $E$ (like electric field, by analogy with the Coulomb case). In view of the discussion of the previous subsection, it is no surprise that $E$ will solve a relation of the form

$$
-\operatorname{div}\left(|y|^{\gamma} E\right)=c_{d, s}\left(\sum_{p \in \Lambda} N_{p} \delta_{p}-m(x) \delta_{\mathbb{R}^{d}}\right) \quad \text { in } \mathbb{R}^{d+k},
$$

where $\Lambda$ is some discrete set in $\mathbb{R}^{d} \times\{0\}$ (identified with $\mathbb{R}^{d}$ ), $N_{p}$ are positive integers, and $m(x)$ is to be specified. For any such $E$ (defined over $\mathbb{R}^{d+k}$ or over subsets of it), we define

$$
E_{\eta}:=E-\sum_{p \in \Lambda} N_{p} \nabla f_{\eta}(x-p)
$$

If $E$ happens to be the gradient of a function $h$, then we will also denote

$$
h_{\eta}:=h-\sum_{p \in \Lambda} N_{p} f_{\eta}(x-p) .
$$

We will write $\Phi_{\eta}$ for the map that sends $E$ to $E_{\eta}$, and note that it is a bijection from the set of vector fields satisfying a relation of the form (1.28) to those satisfying a relation of the form

$$
-\operatorname{div}\left(|y|^{\gamma} E_{\eta}\right)=c_{d, s}\left(\sum_{p \in \Lambda} N_{p} \delta_{p}^{(\eta)}-m(x) \delta_{\mathbb{R}^{d}}\right) \quad \text { in } \mathbb{R}^{d+k}
$$

Remark 1.1. If $h=g *\left(\sum_{i=1}^{n} \delta_{x_{i}}-m(x) \delta_{\mathbb{R}^{d}}\right)$, then the transformation from $h$ to $h_{\eta}$ amounts to truncating the kernel $g$, but only for the Dirac part of the right-hand side. Indeed, letting $g_{\eta}(x)=\min (g(x), g(\eta))$ be the truncated kernel, we have

$$
h_{\eta}=g_{\eta} *\left(\sum_{i=1}^{n} \delta_{x_{i}}\right)-g *\left(m \delta_{\mathbb{R}^{d}}\right) .
$$

Definition 1.2 (Admissible vector fields). Given a number $m \geqslant 0$, we define the class $\mathcal{A}_{m}$ to be the class of gradient vector fields $E=\nabla h$ that satisfy

$$
-\operatorname{div}\left(|y|^{\gamma} \nabla h\right)=c_{d, s}\left(\sum_{p \in \Lambda} N_{p} \delta_{p}-m \delta_{\mathbb{R}^{d}}\right) \quad \text { in } \mathbb{R}^{d+k},
$$

where $\Lambda$ is a discrete set of points in $\mathbb{R}^{d} \times\{0\}$ and $N_{p}$ are integers in $\mathbb{N}^{*}$.

This class corresponds to vector fields that will be limits of blown-ups of those generated by the original configuration $\left(x_{1}, \ldots, x_{n}\right)$, after blow-up at the scale $n^{1 / d}$ near the point 
$x$ where $m=\mu_{V}(x)$ and can be understood as the local density of points. We note that, since such vector fields blow up exactly in $1 /|X|^{s+1}$ near each $p \in \Lambda$ (with the convention $s=0$ for cases (1.3)-(1.4)), such vector fields naturally belong to the space $L_{\text {loc }}^{p}\left(\mathbb{R}^{d+k}, \mathbb{R}^{d+k}\right)$ for $p<\frac{d+k}{s+1}$. In fact, to ensure stability in that class, we will need to take $p<\min \left(2, \frac{2}{\gamma+1}, \frac{d+k}{s+1}\right)$.

We are now in a position to define the renormalized energy. In the definition, we let $K_{R}$ denote the hypercubes $[-R / 2, R / 2]^{d}$.

Definition 1.3 (Renormalized energy). For $\nabla h \in \mathcal{A}_{m}$ and $0<\eta<1$, we define

$$
\mathcal{W}_{\eta}(\nabla h)=\limsup _{R \rightarrow \infty}\left(\frac{1}{R^{d}} \int_{K_{R} \times \mathbb{R}^{k}}|y|^{\gamma}\left|\nabla h_{\eta}\right|^{2}-m c_{d, s} g(\eta)\right)
$$

and

$$
\mathcal{W}(\nabla h)=\lim _{\eta \rightarrow 0} \mathcal{W}_{\eta}(\nabla h)
$$

This is a generalization of the renormalized energy defined in [44]. As in [44], it differs from the one defined in $[47,49]$ for the one-dimensional and two-dimensional logarithmic interaction, essentially in the fact that the order of the limits $R \rightarrow \infty$ and $\eta \rightarrow 0$ is reversed. We refer to [44] for a further discussion of the comparison between the two.

By scaling, we may always reduce to studying the class $\mathcal{A}_{1}$; indeed, if $E \in \mathcal{A}_{m}$, then $\hat{E}=m^{-\frac{s+1}{d}} E\left(\cdot m^{-1 / d}\right) \in \mathcal{A}_{1}^{2}$ and

$$
\mathcal{W}_{\eta}(E)=m^{1+s / d} \mathcal{W}_{\eta m^{1 / d}}(\hat{E}) \quad \mathcal{W}(E)=m^{1+s / d} \mathcal{W}(\hat{E}) .
$$

in case (1.2), and respectively

$$
\mathcal{W}_{\eta}(E)=m\left(\mathcal{W}_{m \eta}(\hat{E})-\frac{2 \pi}{d} \log m\right) \quad \mathcal{W}(E)=m\left(\mathcal{W}(\hat{E})-\frac{2 \pi}{d} \log m\right)
$$

in cases (1.3)-(1.4).

We denote

$$
\xi_{d, s}=\frac{1}{c_{d, s}} \min _{\mathcal{A}_{1}} \mathcal{W}
$$

The name renormalized energy (originating in Bethuel, Brezis, and Hélein's work [6] in the context of two-dimensional Ginzburg-Landau vortices) reflects the fact that $\int|y|^{\gamma}|\nabla h|^{2}$, which is infinite, is computed in a renormalized way by first changing $h$ into $h_{\eta}$ and then removing the appropriate divergent part $c_{d, s} g(\eta)$ per point.

We will prove the following facts about $\mathcal{W}$.

Proposition 1.4 (Minimization of $\mathcal{W}$ ).

1. The limit in (1.34) exists.

2. $\left\{\mathcal{W}_{\eta}\right\}_{\eta<1}$ are uniformly bounded below on $\mathcal{A}_{1}$ by a finite constant depending only on $s$ and $d$.

\footnotetext{
${ }^{2}$ With the convention $s=0$ in case (1.3).
} 
3. $\mathcal{W}$ and $\mathcal{W}_{\eta}$ have a minimizer over the class $\mathcal{A}_{1}$.

4. There exists a minimizing sequence for $\mathcal{W}$ (respectively $\mathcal{W}_{\eta}$ ) formed of periodic configurations (in $\mathbb{R}^{d}$ ) with period $N \rightarrow \infty$.

We can also note that $\mathcal{W}$ does not feel compact perturbations of the points in $\Lambda$. As already mentioned, the question of identifying $\min _{\mathcal{A}_{1}} \mathcal{W}$ is open, and we expect some (Bravais) lattice configuration to achieve the minimum. In view of Proposition 1.4, to identify the value of the minimum it would suffice to compute $\min \mathcal{W}$ over periodic configurations with larger and larger period, for which we have an explicit formula.

Proposition 1.5 (Periodic case). Let $a_{1}, \ldots, a_{N}$ be $N$ points, possibly with repetition, in a torus $\mathbb{T}$ of volume $N$ in $\mathbb{R}^{d}$, for $d \geqslant 1$. On $\mathbb{R}^{d}$ consider the configuration corresponding to the $\mathbb{T}$-periodic repetition of the configuration $a_{1}, \ldots, a_{N}$. Then the following hold.

1. If the points $a_{1}, \ldots, a_{N}$ are not distinct, then for all $E$ such that

$$
-\operatorname{div}\left(|y|^{\gamma} E\right)=c_{d, s}\left(\sum_{i=1}^{N} \delta_{a_{i}}-\delta_{\mathbb{R}^{d}}\right) \quad \text { in } \mathbb{T} \times \mathbb{R}^{k}
$$

(we call such $E$ compatible with the points) it holds that $\mathcal{W}(E)=+\infty$.

2. If all points are distinct, then let $H$ be the function satisfying

$$
-\operatorname{div}\left(|y|^{\gamma} \nabla H\right)=c_{d, s}\left(\sum_{i=1}^{N} \delta_{a_{i}}-\delta_{\mathbb{R}^{d}}\right) \text { in } \mathbb{T} \times \mathbb{R}^{k}, \quad \int_{\mathbb{T} \times\{0\}} H=0 .
$$

Then for any vector field $E=\nabla h$ compatible with $a_{1}, \ldots, a_{N}$ it holds that

$$
\mathcal{W}(E) \geqslant \mathcal{W}(\nabla H)
$$

with equality precisely when $E=\nabla H$. Moreover, it holds that

$$
\mathcal{W}(\nabla H)=\frac{c_{d, s}^{2}}{N} \sum_{i \neq j} G\left(a_{i}-a_{j}\right)+c_{d, s}^{2} \lim _{x \rightarrow 0}\left(G(x)-\frac{g(x)}{c_{s, d}}\right),
$$

where $G$ satisfies

$$
(-\Delta)^{\alpha} G=\delta_{0}-\frac{1}{|\mathbb{T}|} \quad \text { in } \mathbb{T} \quad \int_{\mathbb{T}} G=0,
$$

where $\alpha=\frac{1}{2}(2-\gamma-k)$.

3. In the case when $d=1$ we have

$$
G(x)=2 \frac{N^{2 \alpha-1}}{(2 \pi)^{2 \alpha} \Gamma(2 \alpha)} \int_{0}^{\infty} \frac{t^{2 \alpha-1}\left(e^{t} \cos \left(\frac{2 \pi}{N} x\right)-1\right)}{1-2 e^{t} \cos \left(\frac{2 \pi}{N} x\right)+e^{2 t}} d t .
$$

In the periodic case, $\mathcal{W}$ can thus be seen as a function of the points rather than of the vector fields $E$, via formula (1.38). We note that it would suffice to check that $G$ given by (1.40) is convex to apply the same proof as in [49] to obtain that the minimum of $\mathcal{W}$ in dimension 1 is achieved over the class $\mathcal{A}_{1}$ by the lattice $\mathbb{Z}$. We did not pursue this.

Moreover, specializing (1.38) to the case of Bravais lattice configurations (i.e. $N=1$ ), we are able to prove the analog of the result of [47] in dimension 2. 
Theorem 3. Assume that $d=2$. For any $0<s<2$, the minimum of $\mathcal{W}$ (seen as a function of the point configuration via (1.38)) over lattices $\Lambda=\vec{a} \mathbb{Z}+\vec{b} \mathbb{Z}$ of volume 1 (i.e. such that $\operatorname{det}(\vec{a}, \vec{b})=1$ ) is uniquely achieved (up to rotation) by the triangular lattice, i.e. the one for which $\vec{a}=\left(\frac{\sqrt{3}}{2}\right)^{-1 / 2}(1,0)$ and $\vec{b}=\left(\frac{\sqrt{3}}{2}\right)^{-1 / 2}\left(\frac{1}{2}, \frac{\sqrt{3}}{2}\right)$.

As in [47], the proof consists in connecting the minimization to that of finding the minimizer over lattices of volume 1 of the Epstein zeta function of the lattice $\Lambda, \zeta_{\Lambda}(s)=$ $\sum_{p \in \Lambda \backslash\{0\}} \frac{1}{|p|^{s}}$, which was proved in $[16,24,25,39,42]$ to be the triangular lattice.

\subsection{Connection to the original problem and splitting formula}

We reproduce here the framework of $[44,48,49]$. The renormalized energy will appear as a next order limit of $H_{n}$ after a blow-up is performed, at the inverse of the typical nearest neighbor distance between the points, i.e. $n^{1 / d}$. It is expressed in terms of the potential generated by the configuration $x_{1}, \ldots, x_{n}$, and defined by

$$
h_{n}(X)=g *\left(\sum_{i=1}^{n} \delta_{\left(x_{i}, 0\right)}-n \mu_{V} \delta_{\mathbb{R}^{d}}\right) .
$$

For the blown-up quantities we will use the following notation (with the convention $s=0$ in cases (1.3) or (1.4)):

$$
\begin{aligned}
& x^{\prime}=n^{1 / d} x \quad X^{\prime}=n^{1 / d} X \quad x_{i}^{\prime}=n^{1 / d} x_{i} \\
& \mu_{V}^{\prime}\left(x^{\prime}\right)=\mu_{V}(x) \\
& h_{n}^{\prime}\left(X^{\prime}\right)=n^{-\frac{s}{d}} h_{n}(X) .
\end{aligned}
$$

We note that, in view of (1.41), (1.17), and (1.19), $h_{n}$ and $h_{n}^{\prime}$ satisfy

$$
\begin{aligned}
& -\operatorname{div}\left(|y|^{\gamma} \nabla h_{n}\right)=c_{d, s}\left(\sum_{i=1}^{n} \delta_{x_{i}}-n \mu_{V} \delta_{\mathbb{R}^{d}}\right) \quad \text { in } \mathbb{R}^{d+k}, \\
& -\operatorname{div}\left(|y|^{\gamma} \nabla h_{n}^{\prime}\right)=c_{d, s}\left(\sum_{i=1}^{n} \delta_{x_{i}^{\prime}}-\mu_{V}^{\prime} \delta_{\mathbb{R}^{d}}\right) \quad \text { in } \mathbb{R}^{d+k},
\end{aligned}
$$

while in view of (1.27), $h_{n, \eta}$ and $h_{n, \eta}^{\prime}$, defined from $h_{n}$ and $h_{n}^{\prime}$ via (1.30), satisfy

$$
\begin{aligned}
& -\operatorname{div}\left(|y|^{\gamma} \nabla h_{n, \eta}\right)=c_{d, s}\left(\sum_{i=1}^{n} \delta_{x_{i}}^{(\eta)}-n \mu_{V} \delta_{\mathbb{R}^{d}}\right) \quad \text { in } \mathbb{R}^{d+k}, \\
& -\operatorname{div}\left(|y|^{\gamma} \nabla h_{n, \eta}^{\prime}\right)=c_{d, s}\left(\sum_{i=1}^{n} \delta_{x_{i}^{\prime}}^{(\eta)}-\mu_{V}^{\prime} \delta_{\mathbb{R}^{d}}\right) \quad \text { in } \mathbb{R}^{d+k},
\end{aligned}
$$

with the usual embedding of $\mathbb{R}^{d}$ into $\mathbb{R}^{d+k}$.

The next proposition connects $H_{n}$ with these quantities via an algebraic identity. 
Proposition 1.6 (Splitting formula). For any $n$, and any $x_{1}, \ldots, x_{n}$ distinct points in $\mathbb{R}^{d} \times\{0\}$, letting $h_{n}$ be as in (1.41) and $h_{n, \eta}$ deduced from it via (1.30), we have in case (1.2)

$$
\begin{aligned}
H_{n}\left(x_{1}, \ldots, x_{n}\right)= & n^{2} \mathcal{E}\left(\mu_{V}\right)+2 n \sum_{i=1}^{n} \zeta\left(x_{i}\right) \\
& +n^{1+\frac{s}{d}} \lim _{\eta \rightarrow 0} \frac{1}{c_{d, s}}\left(\frac{1}{n} \int_{\mathbb{R}^{d+k}}|y|^{\gamma}\left|\nabla h_{n, \eta}^{\prime}\right|^{2}-c_{d, s} g(\eta)\right),
\end{aligned}
$$

respectively in the cases (1.3)-(1.4)

$$
\begin{aligned}
H_{n}\left(x_{1}, \ldots, x_{n}\right)= & n^{2} \mathcal{E}\left(\mu_{V}\right)+2 n \sum_{i=1}^{n} \zeta\left(x_{i}\right)-\frac{n}{d} \log n \\
& +n \lim _{\eta \rightarrow 0} \frac{1}{c_{d, s}}\left(\frac{1}{n} \int_{\mathbb{R}^{d+k}}|y|^{\gamma}\left|\nabla h_{n, \eta}^{\prime}\right|^{2}-c_{d, s} g(\eta)\right) .
\end{aligned}
$$

Recalling that $\zeta$ defined in $(1.11)$ is nonnegative and 0 in $\Sigma$ (it acts like an effective potential whose only role is to confine the points to $\Sigma$ ), we see that this formula easily allows us to get a next order lower bound for $H_{n}$, and that it remains to take the $n \rightarrow \infty$ limit in the parentheses in the right-hand side of (1.49), which will lead to $\mathcal{W}$.

In order to make this rigorous, we need to introduce a $\mathcal{W}$ at the level of the 'electric field process', and a way of averaging $\mathcal{W}$ with respect to blow-up centers in $\Sigma$.

More precisely, given any configuration $\mathbf{x}_{n}=\left(x_{1}, \ldots, x_{n}\right)$, we denote $v_{n}=\sum_{i=1}^{n} \delta_{x_{i}}$. The configuration generates (at the blown-up scale) an electric field $E_{v_{n}}$ given by $\nabla h_{n}^{\prime}\left(X^{\prime}\right)$ above. As already mentioned, such electric fields naturally live in the spaces of vector fields $L_{\text {loc }}^{p}\left(\mathbb{R}^{d+k}, \mathbb{R}^{d+k}\right)$ for $p \in\left[1, \min \left(2, \frac{2}{\gamma+1}, \frac{d+k}{s+1}\right)\right)$. Choosing once and for all such a $p$, we define $\mathcal{X}:=\Sigma \times L_{\text {loc }}^{p}\left(\mathbb{R}^{d+k}, \mathbb{R}^{d+k}\right)$ to be the space of 'marked' electric fields, where the mark $x \in \Sigma$ corresponds to the point where we center the blow-up. We denote by $\mathcal{P}(\mathcal{X})$ the space of probability measures on $\mathcal{X}$ endowed with the topology of weak convergence, which can be seen as the space of random marked vector fields.

We may now naturally associate to each configuration $\mathbf{x}_{n}=\left(x_{1}, \ldots, x_{n}\right)$ a 'marked electric field process' $P_{v_{n}}$ via the map

$$
\begin{gathered}
i_{n}: \quad\left(\mathbb{R}^{d}\right)^{n} \longrightarrow \mathcal{P}(\mathcal{X}) \\
\mathbf{x}_{n} \mapsto P_{v_{n}}:=f_{\Sigma} \delta_{\left(x, E_{v_{n}}\left(n^{1 / d} x+\cdot\right)\right)} d x,
\end{gathered}
$$

i.e. $P_{v_{n}}$ is the push-forward of the normalized Lebesgue measure on $\Sigma$ by $x \mapsto$ $\left(x, E_{v_{n}}\left(n^{1 / d} x+\cdot\right)\right)$. Another way of saying this is that each $P_{v_{n}}(x, \cdot)$ is equal to a Dirac at the electric field generated by $\mathbf{x}_{n}$, after centering at the point $x$.

The nice feature is that, under an energy bound on the sequence $\left(\mathbf{x}_{n}\right)_{n}$, the sequence $\left\{P_{v_{n}}\right\}_{n}$ will be tight as $n \rightarrow \infty$, and will thus converge to an element $P$ of $\mathcal{P}(\mathcal{X})$. In a probabilistic point of view, $P$ is a marked electric field process. In an analysis point of view, $P$ is similar to a Young measure on micropatterns formed by the configuration, as for example in [1].

By construction, such a $P=\lim _{n \rightarrow \infty} P_{v_{n}}$ satisfies three properties, which are summarized in the following definition. 
Definition 1.7 (Admissible probabilities). We say that $P \in \mathcal{P}(\mathcal{X})$ is admissible if the following hold.

- The first marginal of $P$ is the normalized Lebesgue measure on $\Sigma$.

- It holds for $P$-a.e. $(x, E)$ that $E \in \mathcal{A}_{\mu_{V}(x)}$.

- $P$ is $T_{\lambda(x)}$-invariant.

Here $T_{\lambda(x)}$-invariant is a strengthening of translation invariance, related to the marking.

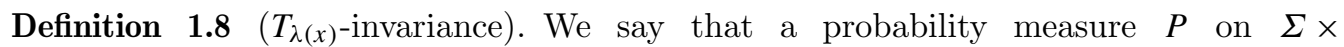
$L_{\text {loc }}^{p}\left(\mathbb{R}^{d+k}, \mathbb{R}^{d+k}\right)$ is $T_{\lambda(x)}$-invariant if $P$ is invariant by $(x, E) \mapsto(x, E(\lambda(x)+\cdot))$, for any $\lambda(x)$ of class $C^{1}$ from $\Sigma$ to $\mathbb{R}^{d}$.

Note that, from such an admissible electric field process, using that $E$ solves (1.32) one can immediately get a (marked) point process by taking the push-forward of $P(x, E)$ by $E \mapsto \frac{1}{c_{s, d}} \operatorname{div}\left(|y|^{\gamma} E\right)+\mu_{V}(x) \delta_{\mathbb{R}^{d}}$.

For each $P \in \mathcal{P}(\mathcal{X})$, we may then define

$$
\tilde{\mathcal{W}}(P)=\frac{|\Sigma|}{c_{d, s}} \int \mathcal{W}(E) d P(x, E)
$$

if $P$ is admissible, and $+\infty$ otherwise.

With these definitions at hand, we may state our main result on minimizers of $H_{n}$ which improves Theorem 1. It identifies the next order $\Gamma$-limit (in the sense of $\Gamma$-convergence) of $H_{n}$ and allows a description of the minimizers at the microscopic level. Below, we abuse notation by writing $v_{n}=\sum_{i=1}^{n} \delta_{x_{i}}$ when it should be $v_{n}=\sum_{i=1}^{n} \delta_{x_{i, n}}$.

Theorem 4 (Microscopic behavior of Riesz energy minimizers). Let the potential $V$ satisfy assumptions (1.8)-(1.10) and (1.12)-(1.14). Let $\left(x_{1}, \ldots, x_{n}\right)$ minimize $H_{n}$, and let $P_{v_{n}}$ be associated via (1.52). Then any subsequence of $\left\{P_{v_{n}}\right\}_{n}$ has a convergent subsequence converging as $n \rightarrow \infty$ to an admissible probability measure $P \in \mathcal{P}(\mathcal{X})$ and, in case (1.2),

$$
\lim _{n \rightarrow \infty} n^{-1-s / d}\left(H_{n}\left(x_{1}, \ldots, x_{n}\right)-n^{2} \mathcal{E}\left(\mu_{V}\right)\right)=\tilde{\mathcal{W}}(P)=\min _{P \text { admissible }} \tilde{\mathcal{W}}=\xi_{d, s} \int \mu_{V}^{1+s / d},
$$

respectively in cases (1.3)-(1.4)

$$
\begin{aligned}
& \lim _{n \rightarrow \infty} n^{-1}\left(H_{n}\left(x_{1}, \ldots, x_{n}\right)-n^{2} \mathcal{E}\left(\mu_{V}\right)+\frac{n}{d} \log n\right) \\
& \quad=\tilde{\mathcal{W}}(P)=\min _{P \text { admissible }} \tilde{\mathcal{W}}=\xi_{d, 0}-\frac{1}{d} \int \mu_{V} \log \mu_{V} ;
\end{aligned}
$$

and $P$ is a minimizer of $\tilde{\mathcal{W}}$, and $E$ minimizes $\mathcal{W}$ over $\mathcal{A}_{\mu_{V}}(x)$ for $P$-a.e. $(x, E)$.

Thus, our result reduces the original question to the minimization of $\widetilde{\mathcal{W}}$, and hence that of $\mathcal{W}$, which we have already discussed. If one believes in the conjecture that minimizers of $\mathcal{W}$ are Bravais lattices, then from the formal statement ' $E$ minimizes $\mathcal{W}$ over $\mathcal{A}_{\mu_{V}(x)}$ ' it 
can be expected that, after zooming at the right scale around $x$, minimizing configurations look like a crystal with the appropriate density $\mu_{V}(x)$.

We conclude our introduction with the two additional results. The first one states that minimizers have points that are well separated at the expected scale of their typical distance, i.e. $n^{-1 / d}$. We provide a short proof of this result based solely on the extension representation and maximum principle arguments. This is to be compared with the analogous statements in [11] for points on the sphere, which rely on fine potential theory arguments.

Theorem 5 (Point separation). Assume that $V$ satisfies (1.8)-(1.10) and that $\mu_{V}$ has a density which satisfies $\left\|\mu_{V}\right\|_{L^{\infty}} \leqslant \bar{m}$. Let $\left(x_{1}, \ldots, x_{n}\right)$ minimize $H_{n}$. Then for each $i \in$ $[1, n], x_{i} \in \Sigma$, and for each $i \neq j$, it holds that

$$
\left|x_{i}-x_{j}\right| \geqslant \frac{r}{(n \bar{m})^{1 / d}}
$$

where $r$ is some positive constant depending only on $s$ and $d$.

The last result concerns the application to statistical mechanics. As in [44, 48, 49], our method can be used to obtain as well next order information on such a system of particles with temperature. More precisely, let us consider the Gibbs measure

$$
d \mathbb{P}_{n, \beta}\left(x_{1}, \ldots, x_{n}\right)=\frac{1}{Z_{n, \beta}} e^{-\beta H_{n}\left(x_{1}, \ldots, x_{n}\right)} d x_{1} \ldots, d x_{n},
$$

where $\beta>0$ is an inverse temperature and $Z_{n, \beta}$ is the partition function of the system, i.e. a number that normalizes $\mathbb{P}_{n, \beta}$ to a probability measure on $\left(\mathbb{R}^{d}\right)^{n}$. Then, under the additional assumption that there exists $\beta_{1}>0$ such that

$$
\begin{cases}\int e^{-\beta_{1} V(x) / 2} d x<\infty & \text { in the case }(1.2) \\ \int e^{-\beta_{1}\left(\frac{V(x)}{2}-\log |x|\right)} d x<\infty & \text { in the cases }(1.3)-(1.4)\end{cases}
$$

we can obtain the following.

Theorem 6 (System with temperature). Assume all the previous assumptions on $V$, i.e. (1.8)-(1.9), (1.12)-(1.14), and (1.57). Let $\bar{\beta}:=\lim _{\sup _{n \rightarrow+\infty}} \beta n^{s / d}{ }^{3}$ and assume that $0<\bar{\beta} \leqslant+\infty$. There exists $C_{\bar{\beta}}>0$ depending only on $V$, $d$, and $s$, with $\lim _{\beta \rightarrow \infty} C_{\bar{\beta}}=0$ and $C_{\infty}=0$ such that the following hold.

1 .

$$
\limsup _{n \rightarrow \infty} n^{-1-s / d}\left|-\frac{\log Z_{n, \beta}}{\beta}-n^{2} \mathcal{E}\left(\mu_{V}\right)-n^{1+s / d} \min \tilde{\mathcal{W}}\right| \leqslant C_{\bar{\beta}},
$$

respectively in the cases (1.3)-(1.4)

$$
\limsup _{n \rightarrow \infty} n^{-1}\left|-\frac{\log Z_{n, \beta}}{\beta}-n^{2} \mathcal{E}\left(\mu_{V}\right)+\frac{n}{d} \log n-n \min \tilde{\mathcal{W}}\right| \leqslant C_{\bar{\beta}} .
$$

${ }^{3}$ Always with the convention $s=0$ in case (1.3). 
2. For fixed $\beta>0$, letting $\widetilde{\mathbb{P}_{n, \beta}}$ denote the push-forward of $\mathbb{P}_{n, \beta}$ by $i_{n}$ (defined in $(1.51)),\left\{\widetilde{\mathbb{P}_{n, \beta}}\right\}_{n}$ is tight and converges as $n \rightarrow \infty$, up to a subsequence, to a probability measure on $\mathcal{P}(\mathcal{X})$ which is concentrated on admissible probabilities satisfying $\widetilde{\mathcal{W}}(P) \leqslant \min \widetilde{\mathcal{W}}+C_{\bar{\beta}}$.

We could also express our results in terms of next order correction to mean-field theory exactly as in [44]. This extension is left to the reader.

To our knowledge, there is no such result in the literature beyond the much-studied case of systems with logarithmic interactions in one and two dimensions, also called $\beta$-ensembles (see [49] and [48] for the references).

This theorem indicates that there is a transition temperature regime $\beta \gg n^{-s / d}$ beyond which the system tends to concentrate on minimizers of $\tilde{\mathcal{W}}$, and hence should be expected to crystallize. For lower $\beta$, in contrast, it is expected that temperature creates disorder.

The rest of the paper is organized as follows. We start by proving the splitting formula and preliminaries on $\mathcal{W}$. In particular we show that $\mathcal{W}_{\eta}$ is essentially monotone in $\eta$. This fact directly provides a uniform lower bound on $\mathcal{W}_{\eta}$. In $\S 3$, we study the periodic case and prove Proposition 1.5 and Theorem 3. In $\S 4$, we prove Theorem 5 and an analogous separation result for minimizers of $\mathcal{W}$ with periodic boundary condition. In $\S 5$, we show the general next order lower bound corresponding to the main result, using the approach based on the ergodic theorem initiated in [47]. Section 6 is devoted to the proof of the general screening result. In $\S 7$, using the screening result we make the construction that allows us to obtain the upper bound for the minimal energy.

\section{Splitting formula and preliminaries on $\mathcal{W}$}

In the whole paper, whenever possible we treat all the cases (1.22)-(1.24) in one unified way. We thus need to carry the extension dimension as $k=0$ or 1 . We note that, in $\S \S 2-5$, we only use weak assumptions on $\mu_{V}$ : that $\mu_{V}$ has an $L^{\infty}$ density, and that it is continuous at almost every point of $\Sigma$. In the statements of all these sections where the parameter $s$ appears, the convention is that $s$ should be taken to be 0 in cases (1.3)-(1.4).

\subsection{Proof of the splitting formula}

Proof. We let $v_{n}=\sum_{i=1}^{n} \delta_{x_{i}}$. Denoting by $\triangle$ the diagonal in $\mathbb{R}^{d} \times \mathbb{R}^{d}$, we may write

$$
\begin{aligned}
H_{n}\left(x_{1}, \ldots, x_{n}\right)= & \sum_{i \neq j} g\left(x_{i}-x_{j}\right)+n \sum_{i=1}^{n} V\left(x_{i}\right) \\
= & \iint_{\Delta^{c}} g(x-y) d v_{n}(x) d v_{n}(y)+n \int V d v_{n} \\
= & n^{2} \iint_{\triangle^{c}} g(x-y) d \mu_{V}(x) d \mu_{V}(y)+n^{2} \int V d \mu_{V} \\
& +2 n \iint_{\triangle^{c}} g(x-y) d \mu_{V}(x) d\left(v_{n}-n \mu_{V}\right)(y)+n \int V d\left(v_{n}-n \mu_{V}\right) \\
& +\iint_{\triangle^{c}} g(x-y) d\left(v_{n}-n \mu_{V}\right)(x) d\left(v_{n}-n \mu_{V}\right)(y) .
\end{aligned}
$$


We now recall that $\zeta$ was defined in (1.11) by

$$
\zeta=h^{\mu_{V}}+\frac{V}{2}-c=\int g(x-y) d \mu_{V}(y)+\frac{V}{2}-c
$$

and that $\zeta=0$ in $\Sigma$ (with the assumptions we made, one can check that $\zeta$ is continuous, so the q.e. relation can be upgraded to everywhere).

With the help of this we may rewrite the middle line in the right-hand side of (2.1) as

$$
\begin{aligned}
& 2 n \iint_{\Delta^{c}} g(x-y) d \mu_{V}(x) d\left(v_{n}-n \mu_{V}\right)(y)+n \int V d\left(v_{n}-n \mu_{V}\right) \\
& =2 n \int\left(h^{\mu_{V}}+\frac{V}{2}\right) d\left(v_{n}-n \mu_{V}\right)=2 n \int(\zeta+c) d\left(v_{n}-n \mu_{V}\right) \\
& =2 n \int \zeta d v_{n}-2 n^{2} \int \zeta d \mu_{V}+2 n c \int d\left(v_{n}-n \mu_{V}\right)=2 n \int \zeta d v_{n},
\end{aligned}
$$

and this relation can be seen as a relation on integrals on $\mathbb{R}^{d}$ (with the restriction of $\zeta$ to $\mathbb{R}^{d}$ ). The last equality is due to the facts that $\zeta \equiv 0$ on the support of $\mu_{V}$ and that $v_{n}$ and $n \mu_{V}$ have the same mass $n$. We also have to notice that, since $\mu_{V}$ (when seen as a measure on $\mathbb{R}^{2}$ ) has an $L^{\infty}$ density with respect to the Lebesgue measure, it does not charge the diagonal $\Delta$ of $\mathbb{R}^{d} \times \mathbb{R}^{d}$ (whose Lebesgue measure is zero), and we can include it back in the domain of integration. By that same argument, one may recognize in the first line of the right-hand side of $(2.1)$ the quantity $n^{2} \mathcal{E}\left(\mu_{V}\right)$.

We may thus rewrite (2.1) as

$$
\begin{aligned}
H_{n}\left(x_{1}, \ldots, x_{n}\right)= & n^{2} \mathcal{E}\left(\mu_{V}\right)+2 n \sum_{i=1}^{n} \zeta\left(x_{i}\right) \\
& +\iint_{\triangle^{c}} g(x-y) d\left(v_{n}-n \mu_{V}\right)(x) d\left(v_{n}-n \mu_{V}\right)(y) .
\end{aligned}
$$

Next, we show that

$$
\iint_{\triangle^{c}} g(x-y) d\left(v_{n}-n \mu_{V}\right)(x) d\left(v_{n}-n \mu_{V}\right)(y)=\lim _{\eta \rightarrow 0}\left(\frac{1}{c_{d, s}} \int_{\mathbb{R}^{d+k}}|y|^{\gamma}\left|\nabla h_{n, \eta}\right|^{2}-n g(\eta)\right) .
$$

To this aim, we compute the right-hand side of this relation. Let us extend the space dimension by $k$, and choose $R$ such that all the points are in $[-R+1, R-1]^{d}$, and $\eta$ small enough that $2 \eta<\min _{i \neq j}\left|x_{i}-x_{j}\right|$. Since $h_{n, \eta}=h_{n}$ (defined in (1.41)) at distance $\geqslant \eta$ from the points, by Green's formula and (1.47), we have

$$
\begin{aligned}
\int_{[-R, R]^{d+k}}|y|^{\gamma}\left|\nabla h_{n, \eta}\right|^{2} & =\int_{\partial[-R, R]^{d+k}}|y|^{\gamma} h_{n} \frac{\partial h_{n}}{\partial v}-\int_{[-R, R]^{d+k}}|y|^{\gamma} h_{n, \eta} \operatorname{div}\left(|y|^{\gamma} \nabla h_{n, \eta}\right) \\
& =\int_{\partial[-R, R]^{d+k}} h_{n} \frac{\partial h_{n}}{\partial v}+c_{d, s} \int_{[-R, R]^{d+k}} h_{n, \eta}\left(\sum_{i} \delta_{x_{i}}^{(\eta)}-\mu_{V} \delta_{\mathbb{R}^{d}}\right) .
\end{aligned}
$$

It is easy to check that, since the total mass on the right-hand side of (1.45) is $0, h_{n}$ decreases like $g^{\prime}(|X|)$ i.e. like $|X|^{-s-1}$ at infinity, and $\nabla h_{n}$ like $g^{\prime \prime}(|X|)$ i.e. $|X|^{-s-2}$ (with the convention that $s=0$ in the logarithmic cases), and thus in all cases the boundary 
integral, split into $\left(\partial[-R, R]^{d} \times[-R, R]^{k}\right) \cup\left([-R, R]^{d} \times \partial[-R, R]^{k}\right)$, can be bounded by $C R^{\gamma+k-1+d-2 s-3}$, and hence tends to 0 as $R \rightarrow \infty$ in view of (1.16). We thus find

$$
\begin{aligned}
\int_{\mathbb{R}^{d+k}}|y|^{\gamma}\left|\nabla h_{n, \eta}\right|^{2} & =c_{d, s} \int_{\mathbb{R}^{d+k}} h_{n, \eta}\left(\sum_{i=1}^{n} \delta_{x_{i}}^{(\eta)}-\mu_{V} \delta_{\mathbb{R}^{d}}\right) \\
& =c_{d, s} \int_{\mathbb{R}^{d+k}}\left(h_{n}-\sum_{i=1}^{n} f_{\eta}\left(x-x_{i}\right)\right)\left(\sum_{i=1}^{n} \delta_{x_{i}}^{(\eta)}-\mu_{V} \delta_{\mathbb{R}^{d}}\right) .
\end{aligned}
$$

Since $f_{\eta}\left(x-x_{i}\right)=0$ on $\partial B\left(x_{i}, \eta\right)=\operatorname{Supp}\left(\delta_{x_{i}}^{(\eta)}\right)$ and outside of $B\left(x_{i}, \eta\right)$, and since the balls $B\left(x_{i}, \eta\right)$ are disjoint, we may write

$$
\int_{\mathbb{R}^{d+k}}|y|^{\gamma}\left|\nabla h_{n, \eta}\right|^{2}=c_{d, s} \int_{\mathbb{R}^{d+k}} h_{n}\left(\sum_{i=1}^{n} \delta_{x_{i}}^{(\eta)}-\mu_{V} \delta_{\mathbb{R}^{d}}\right)-c_{d, s} \int_{\mathbb{R}^{d+k}} \sum_{i=1}^{n} f_{\eta}\left(x-x_{i}\right) \mu_{V} \delta_{\mathbb{R}^{d}} .
$$

Let us now use (temporarily) the notation $h_{n}^{i}(x)=h_{n}(x)-g\left(x-x_{i}\right)$ (for the potential generated by the distribution bereft of the point $x_{i}$ ). The function $h_{n}^{i}$ is regular near $x_{i}$, and hence $\int h_{n}^{i} \delta_{x_{i}}^{(\eta)} \rightarrow h_{n}^{i}\left(x_{i}\right)$ as $\eta \rightarrow 0$. It follows that

$$
\begin{aligned}
c_{d, s} & \int_{\mathbb{R}^{d+k}} h_{n}\left(\sum_{i=1}^{n} \delta_{x_{i}}^{(\eta)}-\mu_{V} \delta_{\mathbb{R}^{d}}\right)-c_{d, s} \int_{\mathbb{R}^{d+k}} \sum_{i=1}^{n} f_{\eta}\left(x-x_{i}\right) \mu_{V} \delta_{\mathbb{R}^{d}} \\
= & n c_{d, s} g(\eta)+c_{d, s} \sum_{i=1}^{n} h_{n}^{i}\left(x_{i}\right)-c_{d, s} \int_{\mathbb{R}^{d+k}} h_{n} \mu_{V} \delta_{\mathbb{R}^{d}} \\
& +O\left(n\left\|\mu_{V}\right\|_{L^{\infty}}\right) \int_{B(0, \eta)}\left|f_{\eta}\right| \delta_{\mathbb{R}^{d}}+o_{\eta}(1) .
\end{aligned}
$$

In case (1.2) we have $\int_{B(0, \eta)}\left|f_{\eta}\right| \delta_{\mathbb{R}^{d}} \leqslant C \eta^{d-s}$, while in cases (1.3)-(1.4) we have $\int_{B(0, \eta)}\left|f_{\eta}\right| \leqslant \int_{B(0, \eta)} \leqslant \eta^{d}|\log \eta|$, and thus, letting $\eta \rightarrow 0$, we find

$$
\lim _{\eta \rightarrow 0} \frac{1}{c_{d, s}} \int_{\mathbb{R}^{d+k}}|y|^{\gamma}\left|\nabla h_{n, \eta}\right|^{2}-n g(\eta)=\sum_{i=1}^{n} h_{n}^{i}\left(x_{i}\right)-\int_{\mathbb{R}^{d+k}} h_{n} \mu_{V} \delta_{\mathbb{R}^{d}}
$$

Now, from the definitions it is easily seen that

$$
h_{n}^{i}\left(x_{i}\right)=\int_{\mathbb{R}^{d} \backslash\left\{x_{i}\right\}} g\left(x_{i}-y\right) d\left(v_{n}-n \mu_{V}\right)(y),
$$

from which it follows that

$$
\begin{aligned}
& \iint_{\triangle^{c}} g(x-y) d\left(v_{n}-n \mu_{V}\right)(x) d\left(v_{n}-n \mu_{V}\right)(y) \\
& \quad=\sum_{i=1}^{n} \int_{\mathbb{R}^{d} \backslash\left\{x_{i}\right\}} g\left(x_{i}-y\right) d\left(v_{n}-n \mu_{V}\right)(y)-n \int_{\mathbb{R}^{d+k}} h_{n} \mu_{V} \delta_{\mathbb{R}^{d}} \\
& \quad=\sum_{i=1}^{n} h_{n}^{i}\left(x_{i}\right)-n \int_{\mathbb{R}^{d+k}} h_{n} \mu_{V} \delta_{\mathbb{R}^{d}} .
\end{aligned}
$$

In view of (2.7), we conclude that the claim holds. 
The final step is to blow up and note that, using the relation $\gamma=s+2-d-k$ and a change of variables, we have

$$
\int_{\mathbb{R}^{d+k}}|y|^{\gamma}\left|\nabla h_{n, \ell}\right|^{2}=n^{\frac{s}{d}} \int_{\mathbb{R}^{d+k}}|y|^{\gamma}\left|\nabla h_{n, \eta}^{\prime}\right|^{2}
$$

with the convention that $s=0$ in cases (1.3)-(1.4), where $\ell=\eta n^{-1 / d}$. We may thus write

$$
\begin{aligned}
\lim _{\eta \rightarrow 0}\left(\int_{\mathbb{R}^{d+k}}|y|^{\gamma}\left|\nabla h_{n, \eta}\right|^{2}-n c_{d, s} g(\eta)\right) & =\lim _{\eta \rightarrow 0}\left(\int_{\mathbb{R}^{d+k}}|y|^{\gamma}\left|\nabla h_{n, n^{-1 / d} \eta}\right|^{2}-n c_{d, s} g\left(n^{-1 / d} \eta\right)\right) \\
& =\lim _{\eta \rightarrow 0}\left(n^{\frac{s}{d}} \int_{\mathbb{R}^{d+k}}|y|^{\gamma}\left|\nabla h_{n, \eta}^{\prime}\right|^{2}-n c_{d, s} g\left(n^{-1 / d} \eta\right)\right),
\end{aligned}
$$

and, combining with the above and rearranging terms, this completes the proof.

\subsection{Coercivity of $\mathcal{W}$}

In this subsection, we show that $\mathcal{W}$ controls the discrepancy between the number of points in a ball and the (suitably rescaled) volume of the ball. We start with two lemmas.

Lemma 2.1 (Average density of points). If $\nabla h \in \mathcal{A}_{m}$ and $\mathcal{W}_{\eta}(\nabla h)<+\infty$ for some $\eta<1$, then

$$
\lim _{R \rightarrow \infty} \frac{\sum_{p \in \Lambda \cap K_{R}} N_{p}}{R^{d}}=m
$$

Proof. We note that, in the case when $k=0$, the result is proven in [44, Lemma 3.1]. We thus assume in the rest of the proof that $k=1$. We denote $K_{R}=[-R / 2, R / 2]^{d} \times\{0\}$ and $\tilde{K}_{R}=[-R / 2, R / 2]^{d+k}$. We also recall that $E_{\eta}=\nabla h_{\eta}$ satisfies

$$
-\operatorname{div}\left(|y|^{\gamma} E_{\eta}\right)=c_{d, s}\left(\sum_{p \in \Lambda} N_{p} \delta_{p}^{(\eta)}-m \delta_{\mathbb{R}^{d}}\right)
$$

and denote $v=\sum_{p \in \Lambda} N_{p} \delta_{p}$. Since $\mathcal{W}_{\eta}(E)<+\infty$, we have $\int_{K_{R} \times \mathbb{R}^{k}}|y|^{\gamma}\left|E_{\eta}\right|^{2} \leqslant C R^{d}$ for any $R>1$ (where the constant $C$ may depend on $\eta$ ). Thus, by a mean-value argument, we may find $t \in[R-1, R]$ such that

$$
\int_{\partial K_{t} \times \mathbb{R}^{k}}|y|^{\gamma}\left|E_{\eta}\right|^{2} \leqslant \int_{K_{R} \times \mathbb{R}^{k}}|y|^{\gamma}\left|E_{\eta}\right|^{2} \leqslant C R^{d}
$$

and then $L \in[1, \sqrt{R}]$ such that

$$
\int_{K_{R} \times \partial[-L, L]^{k}}|y|^{\gamma}\left|E_{\eta}\right|^{2} \leqslant R^{-1 / 2} \int_{K_{R} \times \mathbb{R}^{k}}|y|^{\gamma}\left|E_{\eta}\right|^{2} \leqslant C R^{d-\frac{1}{2}} .
$$

Let us next integrate $(2.9)$ over $K_{t} \times[-L, L]$ and use Green's theorem to find

$$
\int_{K_{t} \times[-L, L]^{k}} \sum_{p \in \Lambda} N_{p} \delta_{p}^{(\eta)}-m\left|K_{t}\right|=-\frac{1}{c_{d, s}} \int_{\partial\left(K_{t} \times[-L, L]\right)}|y|^{\gamma} E_{\eta} \cdot \vec{v},
$$

where $\vec{v}$ denotes the outer unit normal. Using the Cauchy-Schwarz inequality and (2.10)-(2.11), we deduce that 


$$
\begin{aligned}
& \left|\int_{K_{t} \times[-L, L]^{k}} \sum_{p \in \Lambda} N_{p} \delta_{p}^{(\eta)}-m\right| K_{t} \mid \leqslant C\left(\int_{\partial K_{t} \times[-L, L]^{k}}|y|^{\gamma}\right)^{1 / 2}\left(\int_{\partial K_{t} \times[-L, L]^{k}}|y|^{\gamma}\left|E_{\eta}\right|^{2}\right)^{1 / 2} \\
& \quad+C\left(\int_{K_{t} \times \partial[-L, L]^{k}}|y|^{\gamma}\right)^{1 / 2}\left(\int_{K_{t} \times \partial[-L, L]^{k}}|y|^{\gamma}\left|E_{\eta}\right|^{2}\right)^{1 / 2} \\
& \leqslant C L^{\frac{\gamma+1}{2}} R^{\frac{d-1}{2}} R^{\frac{d}{2}}+C L^{\frac{\gamma}{2}} R^{\frac{d}{2}} R^{\frac{d}{2}-\frac{1}{4}}=o\left(R^{d}\right)
\end{aligned}
$$

as $R \rightarrow \infty$, in view of the bound on $L$ and the fact that $\gamma<1$. Since $\eta<1$, by definition of $v$, and since the $\delta_{p}^{(\eta)}$ are supported in $B(p, \eta)$, we have $v\left(K_{R-2}\right) \leqslant$ $\int_{K_{t} \times[-L, L]^{k}} \sum_{p \in \Lambda} N_{p} \delta_{p}^{(\eta)} \leqslant v\left(K_{R+1}\right)$. The result thus follows from (2.13), after dividing by $R^{d}$ and letting $R \rightarrow \infty$.

Lemma 2.2 (Controlling the discrepancy). Assume that E satisfies a relation of the form

$$
-\operatorname{div}\left(|y|^{\gamma} E\right)=c_{d, s}\left(\sum_{p \in \Lambda} N_{p} \delta_{p}-m(x) \delta_{\mathbb{R}^{d}}\right)
$$

in some subset $U \subset \mathbb{R}^{d+k}$ for some $m \in L^{\infty}(U)$, and let $E_{\eta}$ be associated as in (1.29). Then, for any $0<\eta<1, L>2$, and $a \in \mathbb{R}^{d} \times\{0\}$, denoting $\tilde{B}_{L}(a)=B_{L}(a) \times$ $[-L / 2, L / 2]$, if $\tilde{B}_{2 L}(a) \subset U$ we have ${ }^{4}$

$$
\int_{\tilde{B}_{2 L}(a)}|y|^{\gamma}\left|E_{\eta}\right|^{2} \geqslant C \frac{D(a, L)^{2}}{L^{s}} \min \left(1, \frac{D(a, L)}{L^{d}}\right),
$$

for some $C$ depending only on $d, s$, and $\|m\|_{L^{\infty}}$, where $D(a, L)$ denotes the discrepancy

$$
\sum_{p \in \Lambda \cap B_{L}(a)} N_{p}-\int_{B_{L}(a)} m(x) d x
$$

Proof. The proof follows [44, Lemma 3.8]. For simplicity of notation we denote $\tilde{B}_{t}(a)=\tilde{B}_{t}$. We first consider the case that $D:=D(a, L)>0$. We first note that, if

$$
L+\eta \leqslant t \leqslant T:=\min \left(2 L,\left((L+\eta)^{d}+\frac{D}{2 C}\right)^{\frac{1}{d}}\right)
$$

with $C$ well-chosen, we have

$$
\begin{aligned}
-\int_{\tilde{B}_{t}}|y|^{\gamma} E_{\eta} \cdot \vec{v} & =-\int_{\tilde{B}_{t}} \operatorname{div}\left(|y|^{\gamma} E_{\eta}\right)=c_{d, s} \int_{\tilde{B}_{t}}\left(\sum_{p \in \Lambda} N_{p} \delta_{p}^{(\eta)}-m(x) \delta_{\mathbb{R}^{d}}\right) \\
& \geqslant c_{d, s}\left(D-\int_{B_{t} \backslash B_{L}} m(x) d x\right) \geqslant c_{d, s} D-C\left(t^{d}-L^{d}\right) \geqslant \frac{c_{d, s}}{2} D,
\end{aligned}
$$

${ }^{4}$ With the convention that $s=0$ in cases $(1.3)-(1.4)$ 
if we choose the same $C$ in (2.15). By the Cauchy-Schwarz inequality it holds (with the convention that $s=0$ in cases (1.3)-(1.4)) that

$$
\begin{aligned}
\int_{\tilde{B}_{2 L}}|y|^{\gamma}\left|E_{\eta}\right|^{2} & \geqslant \int_{L+\eta}^{T}\left(\int_{\partial \tilde{K}_{t}}|y|^{\gamma}\right)^{-1}\left(\int_{\partial \tilde{B}_{t}}|y|^{\gamma} E_{\eta} \cdot v\right)^{2} d t \\
& =C D^{2} \int_{L+\eta}^{T} t^{-(\gamma+d+k-1)} d t=C D^{2}(g(L+\eta)-g(T))
\end{aligned}
$$

using the previous estimate and (1.16). Inserting the definition of $T$ and rearranging terms, one easily checks that we obtain (2.14). There remains to treat the case where $D \leqslant 0$. This time, we let

$$
T \leqslant t \leqslant L-\eta, \quad T:=\left((L-\eta)^{d}-\frac{D}{2 C}\right)^{\frac{1}{d}},
$$

and if $C$ is well chosen we have

$$
\begin{aligned}
-\int_{\partial \tilde{B}_{t}}|y|^{\gamma} E_{\eta} \cdot \vec{v} & =-\int_{\tilde{B}_{t}} \operatorname{div}\left(|y|^{\gamma} E_{\eta}\right)=c_{d, s} \int_{\tilde{B}_{t}}\left(\sum_{p \in \Lambda} N_{p} \delta_{p}^{(\eta)}-m(x) \delta_{\mathbb{R}^{d}}\right) \\
& \leqslant c_{d, s}\left(D-\int_{B_{L} \backslash B_{t}} m(x) d x\right) \leqslant \frac{c_{d, s}}{2} D,
\end{aligned}
$$

and the rest of the proof is analogous, integrating from $T$ to $L-\eta$.

\subsection{Monotonicity of $\mathcal{W}_{\eta}$}

Next, we prove that applying the $\eta$ truncation to the energy is essentially monotone in $\eta$ (this is natural if we recall that it is almost truncating the kernel at level $\eta$ ).

Lemma 2.3. For any $x_{1}, \ldots, x_{n} \in \mathbb{R}^{d}$, and any $1>\eta>\alpha>0$, letting $h_{n}^{\prime}, h_{n, \eta}^{\prime}$ be as in (1.44), (1.46), and (1.48), we have

$$
\begin{aligned}
-C n\left\|\mu_{V}\right\|_{L^{\infty}} \eta^{\frac{d-s}{2}} \leqslant & \left(\int_{\mathbb{R}^{d+k}}|y|^{\gamma}\left|\nabla h_{n, \alpha}^{\prime}\right|^{2}-n c_{d, s} g(\alpha)\right)-\left(\int_{\mathbb{R}^{d+k}}|y|^{\gamma}\left|\nabla h_{n, \eta}^{\prime}\right|^{2}-n c_{d, s} g(\eta)\right) \\
\leqslant & C n\left\|\mu_{V}\right\|_{L^{\infty}} \eta^{\frac{d-s}{2}}+c_{d, s} \sum_{i \neq j,\left|x_{i}^{\prime}-x_{j}^{\prime}\right| \leqslant 2 \eta} \min \left(g(\alpha), g\left(\left|x_{i}^{\prime}-x_{j}^{\prime}\right|-\alpha\right)\right) \\
& -g\left(\left|x_{i}^{\prime}-x_{j}^{\prime}\right|+\eta\right),
\end{aligned}
$$

where $C$ depends only on $d$ and $s$.

Proof. As in the introduction, we use the notation $g_{\eta}(x)=\min (g(x), g(\eta))$, and we note that since $g_{\eta}=g-f_{\eta}$ (recall (1.25) and (1.27)) we have

$$
-\operatorname{div}\left(|y|^{\gamma} \nabla g_{\eta}\right)=c_{d, s} \delta_{0}^{(\eta)}
$$

${ }^{5}$ With the convention that $s=0$ in cases (1.3)-(1.4). 
We then let $f_{\alpha, \eta}:=f_{\alpha}-f_{\eta}$. We note that $f_{\alpha, \eta}$ vanishes outside $B(0, \eta)$, and

$$
g(\eta)-g(\alpha) \leqslant g_{\eta}-g_{\alpha}=f_{\alpha, \eta} \leqslant 0,
$$

and $f_{\alpha, \eta}$ solves (cf. (1.27))

$$
-\operatorname{div}\left(|y|^{\gamma} \nabla f_{\alpha, \eta}\right)=c_{d, s}\left(\delta_{0}^{(\eta)}-\delta_{0}^{(\alpha)}\right) .
$$

In view of (1.30), we have $\nabla h_{n, \eta}^{\prime}=\nabla h_{n, \alpha}^{\prime}+\sum_{i=1}^{n} \nabla f_{\alpha, \eta}\left(\cdot-x_{i}^{\prime}\right)$ and

$$
-\operatorname{div}\left(|y|^{\gamma} \nabla h_{n, \alpha}^{\prime}\right)=c_{d, s}\left(\sum_{i=1}^{n} \delta_{x_{i}^{\prime}}^{(\alpha)}-\mu_{V} \delta_{\mathbb{R}^{d}}\right) .
$$

Thus,

$$
\begin{aligned}
\int_{\mathbb{R}^{d+k}}|y|^{\gamma}\left|\nabla h_{n, \eta}^{\prime}\right|^{2}= & \int_{\mathbb{R}^{d+k}}|y|^{\gamma}\left|\nabla h_{n, \alpha}^{\prime}\right|^{2}+\sum_{i, j} \int_{\mathbb{R}^{d+k}}|y|^{\gamma} \nabla f_{\alpha, \eta}\left(X-x_{i}^{\prime}\right) \cdot \nabla f_{\alpha, \eta}\left(X-x_{j}^{\prime}\right) \\
& +2 \sum_{i=1}^{n} \int_{\mathbb{R}^{d+k}}|y|^{\gamma} \nabla f_{\alpha, \eta}\left(X-x_{i}^{\prime}\right) \cdot \nabla h_{n, \alpha}^{\prime}
\end{aligned}
$$

Using (2.18), we first write

$$
\begin{aligned}
& \sum_{i, j} \int_{\mathbb{R}^{d+k}}|y|^{\gamma} \nabla f_{\alpha, \eta}\left(X-x_{i}^{\prime}\right) \cdot \nabla f_{\alpha, \eta}\left(X-x_{j}^{\prime}\right) \\
& =-\sum_{i, j} \int_{\mathbb{R}^{d+k}} f_{\alpha, \eta}\left(X-x_{i}^{\prime}\right) \operatorname{div}\left(|y|^{\gamma} \nabla f_{\alpha, \eta}\left(X-x_{j}^{\prime}\right)\right) \\
& =c_{d, s} \sum_{i, j} \int_{\mathbb{R}^{d+k}} f_{\alpha, \eta}\left(X-x_{i}^{\prime}\right)\left(\delta_{x_{j}^{\prime}}^{(\eta)}-\delta_{x_{j}^{\prime}}^{(\alpha)}\right) .
\end{aligned}
$$

Next, using (2.19), we write

$$
\begin{aligned}
2 \sum_{i=1}^{n} \int_{\mathbb{R}^{d+k}}|y|^{\gamma} \nabla f_{\alpha, \eta}\left(X-x_{i}^{\prime}\right) \cdot \nabla h_{n, \alpha}^{\prime} & =-2 \sum_{i=1}^{n} \int_{\mathbb{R}^{d+k}} f_{\alpha, \eta}\left(X-x_{i}^{\prime}\right) \operatorname{div}\left(|y|^{\gamma} \nabla h_{n, \alpha}^{\prime}\right) \\
& =2 c_{d, s} \sum_{i=1}^{n} \int_{\mathbb{R}^{d+k}} f_{\alpha, \eta}\left(X-x_{i}^{\prime}\right)\left(\sum_{j=1}^{n} \delta_{x_{j}^{\prime}}^{(\alpha)}-\mu_{V^{\prime}}^{\prime} \delta_{\mathbb{R}^{d}}\right) .
\end{aligned}
$$

These last two equations add up to give a right-hand side equal to

$$
\begin{aligned}
& \sum_{i \neq j} c_{d, s} \int_{\mathbb{R}^{d+k}} f_{\alpha, \eta}\left(X-x_{i}^{\prime}\right)\left(\delta_{x_{j}^{\prime}}^{(\alpha)}+\delta_{x_{j}^{\prime}}^{(\eta)}\right)-2 c_{d, s} \sum_{i=1}^{n} \int f_{\alpha, \eta}\left(X-x_{i}^{\prime}\right) \mu_{V}^{\prime} \delta_{\mathbb{R}^{d}} \\
& \quad+n c_{d, s} \int_{\mathbb{R}^{d+k}} f_{\alpha, \eta}\left(\delta_{0}^{(\alpha)}+\delta_{0}^{(\eta)}\right) .
\end{aligned}
$$


We then note that $\int f_{\alpha, \eta}\left(\delta_{0}^{(\alpha)}+\delta_{0}^{(\eta)}\right)=-\int f_{\eta} \delta_{0}^{(\alpha)}=-(g(\alpha)-g(\eta))$ by definition of $f_{\eta}$ and the fact that $\delta_{0}^{(\alpha)}$ is a measure supported on $\partial B(0, \alpha)$ and of mass 1 . Second, we bound $\int_{\mathbb{R}^{d+k}} f_{\alpha, \eta}\left(X-x_{i}^{\prime}\right) \mu_{V}^{\prime} \delta_{\mathbb{R}^{d}}$ by

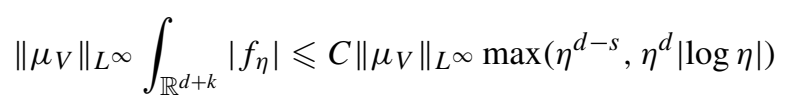

according to the cases, as seen in the proof of the splitting formula. Third, we observe that, in view of (2.17), the first term in (2.23) is nonpositive, and that only the terms for which $\left|x_{i}^{\prime}-x_{j}^{\prime}\right| \leqslant 2 \eta$ contribute. We now bound its absolute value using (2.17) and (2.16):

$$
\begin{aligned}
& \left|\sum_{i \neq j} c_{d, s} \int_{\mathbb{R}^{d+k}} f_{\alpha, \eta}\left(X-x_{i}^{\prime}\right)\left(\delta_{x_{j}^{\prime}}^{(\alpha)}+\delta_{x_{j}^{\prime}}^{(\eta)}\right)\right| \\
& \quad=-\sum_{i \neq j} \int_{\mathbb{R}^{d+k}}\left(g_{\alpha}-g_{\eta}\right)\left(X-x_{i}^{\prime}\right) \operatorname{div}\left(|y|^{\gamma}\left(\nabla g_{\alpha}\left(X-x_{j}^{\prime}\right)+\nabla g_{\eta}\left(X-x_{j}^{\prime}\right)\right)\right) \\
& \quad=\sum_{i \neq j} \int_{\mathbb{R}^{d+k}}|y|^{\gamma}\left(\nabla g_{\alpha}-\nabla g_{\eta}\right)\left(X-x_{i}^{\prime}\right) \cdot\left(\nabla g_{\alpha}+\nabla g_{\eta}\right)\left(X-x_{j}^{\prime}\right) \\
& =\sum_{i \neq j} \int_{\mathbb{R}^{d+k}}|y|^{\gamma} \nabla g_{\alpha}\left(X-x_{i}^{\prime}\right) \cdot \nabla g_{\alpha}\left(X-x_{j}^{\prime}\right)-|y|^{\gamma} \nabla g_{\eta}\left(X-x_{i}^{\prime}\right) \cdot \nabla g_{\eta}\left(X-x_{j}^{\prime}\right),
\end{aligned}
$$

where we noted that the other terms cancel out in the sum when exchanging the roles of $i$ and $j$. Integrating by parts again using (2.16), and using the fact that $\delta_{x}^{(\eta)}$ is a measure of mass 1 supported on $\partial B(x, \eta)$, we are led to

$$
\begin{aligned}
& \left|\sum_{i \neq j} c_{d, s} \int_{\mathbb{R}^{d+k}} f_{\alpha, \eta}\left(X-x_{i}^{\prime}\right)\left(\delta_{x_{j}^{\prime}}^{(\alpha)}+\delta_{x_{j}^{\prime}}^{(\eta)}\right)\right|=c_{d, s} \sum_{i \neq j} \int_{\mathbb{R}^{d+k}} g_{\alpha}\left(X-x_{i}^{\prime}\right) \delta_{x_{j}^{\prime}}^{(\alpha)}-g_{\eta}\left(X-x_{i}^{\prime}\right) \delta_{x_{j}^{\prime}}^{(\eta)} \\
& \quad \leqslant c_{d, s} \sum_{i \neq j,\left|x_{i}^{\prime}-x_{j}^{\prime}\right| \leqslant 2 \eta} g_{\alpha}\left(\left|x_{i}^{\prime}-x_{j}^{\prime}\right|-\alpha\right)-g_{\eta}\left(\left|x_{i}^{\prime}-x_{j}^{\prime}\right|+\eta\right) \\
& \quad \leqslant c_{d, s} \sum_{i \neq j,\left|x_{i}^{\prime}-x_{j}^{\prime}\right| \leqslant 2 \eta} \min \left(g(\alpha), g\left(\left|x_{i}^{\prime}-x_{j}^{\prime}\right|-\alpha\right)\right)-g\left(\left|x_{i}^{\prime}-x_{j}^{\prime}\right|+\eta\right)
\end{aligned}
$$

where we used the fact that $g$ and $g_{\eta}$ are radial decreasing.

We conclude that

$$
\begin{aligned}
- & C n\left\|\mu_{V}\right\|_{L^{\infty}} \max \left(\eta^{d-s}, \eta^{d}|\log \eta|\right) \\
\leqslant & \left(\int|y|^{\gamma}\left|\nabla h_{n, \alpha}^{\prime}\right|^{2}-n c_{d, s} g(\alpha)\right)-\left(\int_{\mathbb{R}^{d+k}}|y|^{\gamma}\left|\nabla h_{n, \eta}^{\prime}\right|^{2}-n c_{d, s} g(\eta)\right) \\
\leqslant & C n\left\|\mu_{V}\right\|_{L^{\infty} \max \left(\eta^{d-s}, \eta^{d}|\log \eta|\right)} \\
& +c_{d, s} \sum_{i \neq j,\left|x_{i}^{\prime}-x_{j}^{\prime}\right| \leqslant 2 \eta} \min \left(g(\alpha), g\left(\left|x_{i}^{\prime}-x_{j}^{\prime}\right|-\alpha\right)\right)-g\left(\left|x_{i}^{\prime}-x_{j}^{\prime}\right|+\eta\right),
\end{aligned}
$$


and this finishes the proof, noting that in all cases we have $\max \left(\eta^{d-s}, \eta^{d}|\log \eta|\right) \leqslant \eta^{\frac{d-s}{2}}$ if one takes the convention that $s=0$ in the logarithmic cases.

The next proposition expresses the same fact at the level of the limits.

Proposition 2.4. Let $E \in \mathcal{A}_{m}$. For any $1>\eta>\alpha>0$ such that $\mathcal{W}_{\alpha}(E)<+\infty$, we have

$$
\mathcal{W}_{\eta}(E) \leqslant \mathcal{W}_{\alpha}(E)+C m^{2} \eta^{\frac{d-s}{2}},
$$

where $C$ depends only on $s$ and $d$; and thus $\lim _{\eta \rightarrow 0} \mathcal{W}_{\eta}(E)=\mathcal{W}(E)$ always exists. Moreover, $\mathcal{W}_{\eta}$ is bounded below on $\mathcal{A}_{m}$ by a constant depending only on $s, d$, and $m$.

We note that this proves items 1 and 2 in Proposition 1.4.

Proof. Let us consider $E$ satisfying a relation of the form

$$
-\operatorname{div}\left(|y|^{\gamma} E\right)=c_{d, s}\left(\sum_{p \in \Lambda} N_{p} \delta_{p}-m(x) \delta_{\mathbb{R}^{d}}\right) \quad \text { in } K_{R} \times \mathbb{R}^{k}
$$

for some $m \in L^{\infty}\left(K_{R}\right)$. Let $E_{\eta}$ be associated via (1.29). Assume that $1>\eta>\alpha>0$. Let $K_{R}=[-R / 2, R / 2]^{d} \times\{0\}$ and $\tilde{K}_{R}=[-R / 2, R / 2]^{d+k}$. Let $\chi_{R}$ denote a smooth cutoff function equal to 1 in $\tilde{K}_{R-3}$ and vanishing outside $\tilde{K}_{R-2}$, with $\left|\nabla \chi_{R}\right| \leqslant 1$. As in the previous proof, we note that

$$
E_{\eta}=E_{\alpha}+\sum_{p \in \Lambda} N_{p} \nabla f_{\alpha, \eta}(x-p)
$$

and insert this expression to expand

$$
\begin{aligned}
& \int_{\mathbb{R}^{d+k}} \chi_{R}|y|^{\gamma}\left|E_{\eta}\right|^{2}-\int \chi_{R}|y|^{\gamma}\left|E_{\alpha}\right|^{2} \\
& =\sum_{p, q \in \Lambda} N_{p} N_{q} \int_{\mathbb{R}^{d+k}} \chi_{R}|y|^{\gamma} \nabla f_{\alpha, \eta}(x-p) \cdot \nabla f_{\alpha, \eta}(x-q) \\
& \quad+2 \sum_{p \in \Lambda} N_{p} \int_{\mathbb{R}^{d+k}}|y|^{\gamma} \chi_{R} \nabla f_{\alpha, \eta}(x-p) \cdot E_{\alpha} .
\end{aligned}
$$

Integrating by parts and using the properties of $\chi_{R}$, we find that

$$
\int_{\mathbb{R}^{d+k}} \chi_{R}|y|^{\gamma}\left|E_{\eta}\right|^{2}-\int_{\mathbb{R}^{d+k}} \chi_{R}|y|^{\gamma}\left|E_{\alpha}\right|^{2}=\text { Error }+ \text { Main }
$$

where

$$
\begin{aligned}
\text { Main }:= & -\sum_{p, q \in \Lambda} N_{p} N_{q} \int_{\mathbb{R}^{d+k}} \chi_{R} f_{\alpha, \eta}(x-p) \operatorname{div}\left(|y|^{\gamma} f_{\alpha, \eta}(x-q)\right) \\
& -2 \sum_{p \in \Lambda} N_{p} \int_{\mathbb{R}^{d+k}} \chi_{R} f_{\alpha, \eta}(x-p) \operatorname{div}\left(|y|^{\gamma} E_{\alpha}\right) .
\end{aligned}
$$




$$
\begin{aligned}
\mid \text { Error } \mid \leqslant & C \sum_{p, q \in\left(K_{R-2} \backslash K_{R-3}\right) \cap \Lambda} N_{p} N_{q} \int_{\mathbb{R}^{d+k}}|y|^{\gamma}\left|\nabla f_{\alpha, \eta}(x-p)\right|\left|f_{\alpha, \eta}(x-q)\right| \\
& +\sum_{p \in\left(K_{R-2} \backslash K_{R-3}\right) \cap \Lambda} N_{p} \int_{\mathbb{R}^{d+k}}|y|^{\gamma}\left|E_{\alpha}\right|\left|f_{\alpha, \eta}(x-p)\right| .
\end{aligned}
$$

We will work on controlling Error just below, and for now, using the fact that $f_{\alpha, \eta}$ is supported in $B(0, \eta)$, we may write, as we did in the proof of Lemma 2.3,

$$
\begin{aligned}
\text { Main }= & -\sum_{p, q \in \Lambda} N_{p} N_{q} \int \chi_{R} f_{\alpha, \eta}(x-p) \operatorname{div}\left(|y|^{\gamma} \nabla f_{\alpha, \eta}(x-q)\right) \\
& -2 \sum_{p \in \Lambda} N_{p} \int \chi_{R} f_{\alpha, \eta}(x-p) \operatorname{div}\left(|y|^{\gamma} E_{\alpha}\right) \\
= & c_{d, s} \sum_{p, q \in \Lambda} N_{p} N_{q} \int \chi_{R} f_{\alpha, \eta}(x-p)\left(\delta_{q}^{(\eta)}+\delta_{q}^{(\alpha)}\right) \\
& -2 c_{d, s} \sum_{p \in \Lambda} N_{p} \int \chi_{R} f_{\alpha, \eta}(x-p) m(x) \delta_{\mathbb{R}^{d}}
\end{aligned}
$$

Using (2.17), we have

$$
\begin{aligned}
2 c_{d, s}(g(\eta)-g(\alpha)) & \leqslant \int f_{\alpha, \eta}(x-p)\left(\delta_{q}^{(\eta)}+\delta_{q}^{(\alpha)}\right) \\
& \leqslant \min \left(0, \int\left(g_{\eta}-g_{\alpha}\right)(x-p) \delta_{q}^{(\alpha)}\right) \leqslant(g(\eta)-g(|p-q|+\alpha))_{-} .
\end{aligned}
$$

Combining these two relations, we obtain

$$
\begin{aligned}
& -C\|m\|_{L^{\infty}} \eta^{\frac{d-s}{2}} \sum_{p \in \Lambda \cap K_{R-2}} N_{p}+2 c_{d, s}(g(\eta)-g(\alpha)) \sum_{p \neq q \in \Lambda \cap K_{R-2},|p-q| \leqslant 2 \eta} N_{p} N_{q} \\
& \leqslant \text { Main }-c_{d, s} \sum_{p \in \Lambda \cap K_{R-2}} N_{p}(g(\eta)-g(\alpha)) \\
& \leqslant C\|m\|_{L^{\infty}} \eta^{\frac{d-s}{2}} \sum_{p \in \Lambda \cap K_{R-3}} N_{p}+c_{d, s} \sum_{p \neq q \in \Lambda \cap K_{R-2}} N_{p} N_{q}(g(\eta)-g(|p-q|+\alpha))_{-} .
\end{aligned}
$$

Next, to control Error, we partition $K_{R-1} \backslash K_{R-4}$ into disjoint cubes $\mathcal{C}_{j}$ of sidelength centered at points $y_{j}$, and we denote $\mathcal{N}_{j}=\sum_{p \in \Lambda \cap \mathcal{C}_{j}} N_{p}$. By Lemma 2.2, we have that $\mathcal{N}_{j}^{2} \leqslant C+C e_{j}$, where

$$
e_{j}:=\int_{B_{2}\left(y_{j}\right) \times(-R / 2, R / 2)^{k}}|y|^{\gamma}\left|E_{\alpha}\right|^{2} .
$$

Using that the overlap of the $B_{2}\left(y_{j}\right)$ is bounded, we may write

$$
\sum_{j} \mathcal{N}_{j}^{2} \leqslant C R^{d-1}+\sum_{j} e_{j} \leqslant C R^{d-1}+\int_{\tilde{K}_{R} \backslash \tilde{K}_{R-5}}|y|^{\gamma}\left|E_{\alpha}\right|^{2} .
$$


We then may deduce, by separating the contributions in each $\mathcal{C}_{j}$, and using the Cauchy-Schwarz inequality and $\int\left|f_{\alpha, \eta}\right|^{2} \leqslant C \eta^{d} g^{2}(\alpha)$, that

$$
\begin{aligned}
\text { Error } & \leqslant C g(\alpha) \sum_{j} \mathcal{N}_{j}^{2}+C \sum_{j} \eta^{d / 2} g(\alpha) \mathcal{N}_{j} e_{j}^{1 / 2} \leqslant C \eta^{d} g^{2}(\alpha)\left(\sum_{j} \mathcal{N}_{j}^{2}+\sum_{j} e_{j}\right) \\
& \leqslant C \eta^{d} g^{2}(\alpha)\left(R^{d-1}+\int_{\tilde{K}_{R} \backslash \tilde{K}_{R-5}}|y|^{\gamma}\left|E_{\alpha}\right|^{2}\right) .
\end{aligned}
$$

Returning to (2.25), we have found that

$$
\begin{aligned}
- & C\|m\|_{L^{\infty}} \eta^{\frac{d-s}{2}} \sum_{p \in \Lambda \cap K_{R-4}} N_{p}+c_{d, s} \sum_{p \neq q \in \Lambda \cap K_{R-3}} N_{p} N_{q}(g(|p-q|+\alpha)-g(\eta))_{+} \\
& -C g(\alpha)\left(R^{d-1}+\int_{\tilde{K}_{R} \backslash \tilde{K}_{R-5}}|y|^{\gamma}\left|E_{\alpha}\right|^{2}\right) \\
\leqslant & \left(\int \chi_{R}|y|^{\gamma}\left|E_{\alpha}\right|^{2}-c_{d, s} \sum_{p \in \Lambda \cap K_{R-4}} N_{p} g(\alpha)\right) \\
& -\left(\int \chi_{R}|y|^{\gamma}\left|E_{\eta}\right|^{2}-c_{d, s} \sum_{p \in \Lambda \cap K_{R-4}} N_{p} g(\eta)\right) \\
\leqslant & 2 c_{d, s}(g(\alpha)-g(\eta)) \quad \sum_{p \neq q \in \Lambda \cap K_{R-2},|p-q| \leqslant 2 \eta} N_{p} N_{q}+C\|m\|_{L^{\infty}} \eta^{\frac{d-s}{2}} \sum_{p \in \Lambda \cap K_{R-4}} N_{p} \\
& +C \eta^{2} g^{2}(\alpha)\left(R^{d-1}+\int_{\tilde{K}_{R} \backslash \tilde{K}_{R-5}}|y|^{\gamma}\left|E_{\alpha}\right|^{2}\right),
\end{aligned}
$$

and it easily follows that

$$
\begin{aligned}
& c_{d, s} \sum_{p \neq q \in \Lambda \cap K_{R-3}} N_{p} N_{q}(g(|p-q|+\alpha)-g(\eta))_{+} \\
& \leqslant\left(\int_{K_{R} \times \mathbb{R}^{k}}|y|^{\gamma}\left|E_{\alpha}\right|^{2}-c_{d, s} \eta^{d} g^{2}(\alpha) \sum_{p \in \Lambda \cap K_{R}} N_{p}\right) \\
&-\left(\int_{K_{R} \times \mathbb{R}^{k}}|y|^{\gamma}\left|E_{\eta}\right|^{2}-c_{d, s} g(\eta) \sum_{p \in \Lambda \cap K_{R}} N_{p}\right)+\text { error } \\
& \leqslant 2 c_{d, s}(g(\alpha)-g(\eta)) \sum_{p \neq q \in \Lambda \cap K_{R-2},|p-q| \leqslant 2 \eta} N_{p} N_{q}
\end{aligned}
$$

with

$$
\begin{aligned}
\text { |error } \mid \leqslant & C \eta^{\frac{d-s}{2}}\|m\|_{L^{\infty}} \sum_{p \in \Lambda \cap K_{R}} N_{p}+C g(\alpha) \sum_{p \in \Lambda \cap\left(K_{R} \backslash K_{R-5}\right)} N_{p} \\
& +C(1+g(\alpha)) \int_{\left(K_{R} \backslash K_{R-5}\right) \times \mathbb{R}^{k}}|y|^{\gamma}\left|E_{\eta}\right|^{2}+|y|^{\gamma}\left|E_{\alpha}\right|^{2},
\end{aligned}
$$

where $C$ depends only on $s$ and $d$. 
Let us now specialize to $E \in \mathcal{A}_{m}$. In view of Lemma 2.1, we have that $\lim _{R \rightarrow \infty} R^{-d} \sum_{p \in \Lambda \cap K_{R}} N_{p}=m$ and $\lim _{R \rightarrow \infty} R^{-d} \sum_{p \in \Lambda \cap\left(K_{R} \backslash K_{R-5}\right)} N_{p}=0$. In addition, since $\mathcal{W}_{\alpha}(E)<\infty$, and by definition of $\mathcal{W}_{\alpha}$, we must have $\lim _{R \rightarrow \infty} R^{-d} \int_{\tilde{K}_{R} \backslash \tilde{K}_{R-5}}|y|^{\gamma}\left|E_{\alpha}\right|^{2}$ $=0$. We deduce that

$$
\begin{aligned}
-C m^{2} \eta^{\frac{d-s}{2}}+\liminf _{R \rightarrow \infty} \frac{1}{R^{d}} c_{d, s} \sum_{p \neq q \in \Lambda \cap K_{R-3}} N_{p} N_{q}(g(|p-q|+\alpha)-g(\eta))_{+} \\
\leqslant \mathcal{W}_{\alpha}(E)-\mathcal{W}_{\eta}(E) \leqslant C m^{2} \eta^{\frac{d-s}{2}} \\
\quad+2 c_{d, s}(g(\alpha)-g(\eta)) \limsup _{R \rightarrow \infty} \frac{1}{R^{d}} \sum_{p \neq q \in \Lambda \cap K_{R-2},|p-q| \leqslant 2 \eta} N_{p} N_{q} .
\end{aligned}
$$

It then immediately follows that $\mathcal{W}_{\eta}$ has a limit (finite or infinite) as $\eta \rightarrow 0$, and that $\mathcal{W}_{\eta}(E)$ is bounded below by, say, $\mathcal{W}_{1 / 2}(E)-C m$, which in view of its definition is obviously bounded below by $-c_{d, s} m-C m$.

\section{The periodic case}

We consider now the case of periodic configurations of charges $\Lambda$ and prove Proposition 1.5 and Theorem 3.

\subsection{Proof of Proposition 1.5}

We start by proving the second point, by a modification of the calculations of the proof of Proposition 1.6. By using the periodicity, we see that we just have to compute

$$
\lim _{\eta \rightarrow 0}\left[\int_{\mathbb{T} \times \mathbb{R}^{k}}|y|^{\gamma}\left|\nabla H_{\eta}\right|^{2}-c_{d, s} N g(\eta)\right] .
$$

We may take $\eta \leqslant \frac{1}{2} \min _{i \neq j}\left|a_{i}-a_{j}\right|$. We remark that

$$
H_{\eta}(x)=\sum_{i=1}^{N}\left(c_{d, s} \bar{G}\left(x-a_{i}\right)-f_{\eta}\left(x-a_{i}\right)\right),
$$

where $\bar{G}$ is the solution in $\mathbb{T} \times \mathbb{R}^{k}$ of

$$
-\operatorname{div}\left(|y|^{\gamma} \nabla \bar{G}\right)=\delta_{0}-\frac{1}{|\mathbb{T}|} \delta_{\mathbb{R}^{d}},
$$

with $\int_{\mathbb{T} \times \mathbb{R}^{k}} \bar{G} \delta_{\mathbb{R}^{d}}=0$. By the extension representation, one may check that the trace $G$ on $\mathbb{R}^{d}$ of $\bar{G}$ solves (1.39). Inserting formula (3.2) into (3.1), we can compute via an integration by parts:

$$
\begin{aligned}
\int_{\mathbb{T} \times \mathbb{R}^{k}}|y|^{\gamma}\left|\nabla H_{\eta}\right|^{2} & =-\int_{\mathbb{T} \times \mathbb{R}^{k}} H_{\eta} \operatorname{div}\left(|y|^{\gamma} \nabla H_{\eta}\right) \\
& =c_{d, s} \int\left(\sum_{i=1}^{N}\left(c_{d, s} H\left(x-a_{i}\right)-f_{\eta}\left(x-a_{i}\right)\right)\right)\left(\sum_{j=1}^{N} \delta_{a_{j}}^{(\eta)}-\delta_{\mathbb{R}^{d}}\right) d x .
\end{aligned}
$$


Now we may continue exactly like in the proof of Proposition 1.6, with $\sum_{i=1}^{n} \bar{G}\left(x-a_{i}\right)$ in place of $h_{n}(x)$ and with 1 in place of $\mu_{V}$. As an analog of $h_{n}^{i}(x)$ we thus have

$$
c_{d, s} \sum_{j \neq i} \bar{G}\left(x-a_{j}\right)+N \lim _{|x| \rightarrow 0}\left(c_{d, s} \bar{G}(x)-g(x)\right) ;
$$

therefore, since $\bar{G}$ has zero average, we obtain the following analog of (2.7), where we write $G$ instead of $\bar{G}$ since all the quantities can be computed on the trace:

$$
\lim _{\eta \rightarrow 0} \frac{1}{c_{d, s}^{2}}\left[\int_{\mathbb{T} \times \mathbb{R}^{k}}|y|^{\gamma}\left|\nabla H_{\eta}\right|^{2}-N c_{d, s} g(\eta)\right]=\sum_{i \neq j} G\left(a_{i}-a_{j}\right)+N \lim _{x \rightarrow 0}\left(G(x)-\frac{g(x)}{c_{s, d}}\right) .
$$

This is equivalent to (1.38). Now assume that $E=\nabla h$ is another periodic vector field (in the first $d$ coordinates) which is compatible with the points $a_{1}, \ldots, a_{N}$. We have that $\nabla u=\nabla h-\nabla H$ solves $\operatorname{div}\left(|y|^{\gamma} \nabla u\right)=0$ and is periodic. We may then write

$$
\int_{\mathbb{T} \times \mathbb{R}^{k}}|y|^{\gamma}\left|(\nabla u+\nabla H)_{\eta}\right|^{2}=\int_{\mathbb{T} \times \mathbb{R}^{k}}|y|^{\gamma}|\nabla u|^{2}+\int_{\mathbb{T} \times \mathbb{R}^{k}}\left|\nabla H_{\eta}\right|^{2},
$$

where the cross-term has vanished by using the periodicity of $H_{\eta}$ and of $\nabla u$, and $\operatorname{div}\left(|y|^{\gamma} \nabla u\right)=0$. It is then straightforward to deduce that $\mathcal{W}(\nabla h) \geqslant \mathcal{W}(\nabla H)$, which finishes the proof of item 2 .

We now turn to the proof of item 1 . Suppose that the points $a_{1}, \ldots, a_{N}$ are not distinct. Without loss of generality, we have $k$ points $a_{1}, \ldots, a_{k}$ with multiplicities $N_{1}, \ldots, N_{k}$ with $\sum_{i=1}^{k} N_{i}=N, \max _{i} N_{i} \geqslant 2$. The left-hand side of (2.6) in our periodic case then contains a first term equal to $c_{d, s} g(\eta) \sum_{i=1}^{k} N_{i}^{2}$, which is not canceled by the negative $c_{d, s} N g(\eta)$ term any more. The remaining terms in (2.6) are respectively positive, zero, and $o_{\eta}(1)$; therefore we have

$$
\int_{\mathbb{T} \times \mathbb{R}}|y|^{\gamma}\left|\nabla H_{\eta}\right|^{2}=c_{d, s} g(\eta)\left(\sum_{i=1}^{k} N_{i}^{2}-N\right)+o_{\eta}(1) \rightarrow+\infty, \quad(\eta \rightarrow 0),
$$

as desired.

For the third item, we generalize the proof of [8, Prop. 2.10]. We solve equation (1.39) satisfied by $G$ by Fourier transform. We choose the following normalization for Fourier transforms and series:

$$
\begin{aligned}
\widehat{f}(\xi) & =\int f(x) e^{-2 i \pi x \cdot \xi} d x \\
c_{k}(f) & =\int_{\mathbb{T}_{N}} f(x) e^{-\frac{2 i \pi k x}{N}} d x .
\end{aligned}
$$

Then the Fourier inversion formula is $f(x)=\int \widehat{f}(\xi) e^{2 i \pi x \cdot \xi} d \xi$, and

$$
f(x)=\frac{1}{N} \sum_{k \in \mathbb{Z}} c_{k}(f) e^{\frac{2 i \pi k}{N} x} .
$$

If $G$ solves (1.39), then $\widehat{G}$ has to satisfy

$$
\left(\frac{2 \pi m}{N}\right)^{2 \alpha} \widehat{G}(m)=\widehat{\delta_{(0,0)}}-\delta_{m}^{0} \frac{1}{N}
$$


with $\delta_{m}^{0}$ by definition equal to 1 if $m=0$ and 0 otherwise. Combining these facts, we obtain

$$
\widehat{G}(m)=\frac{1-\delta_{m}^{0}}{\left(\frac{2 \pi|m|}{N}\right)^{2 \alpha}} \quad \text { for } m \neq 0 .
$$

The undetermination of $\widehat{G(0)}$ corresponds to the fact that $G$ is only determined by (1.39) up to a constant. By Fourier inversion with the above normalization, it follows that

$$
G(x)=2 \frac{N^{2 \alpha-1}}{(2 \pi)^{2 \alpha}} \sum_{k=1}^{\infty} \frac{\cos \left(2 \pi \frac{k}{N} x\right)}{k^{2 \alpha}}+c .
$$

We see that the condition $\int_{\mathbb{T}_{N}} G=0$ gives $c=0$. We next use the following formula found in $[40$, p. 726$]$ :

$$
\sum_{k=1}^{\infty} \frac{\cos k y}{k^{a}}=\frac{1}{\Gamma(a)} \int_{0}^{\infty} \frac{t^{a-1}\left(e^{t} \cos y-1\right)}{1-2 e^{t} \cos y+e^{2 t}} d t .
$$

Applying it to $a=2 \alpha$ and $y=\frac{2 \pi}{N} x$, we obtain

$$
G(x)=2 \frac{N^{2 \alpha-1}}{(2 \pi)^{2 \alpha} \Gamma(2 \alpha)} \int_{0}^{\infty} \frac{t^{2 \alpha-1}\left(e^{t} \cos \left(\frac{2 \pi}{N} x\right)-1\right)}{1-2 e^{t} \cos \left(\frac{2 \pi}{N} x\right)+e^{2 t}} d t
$$

which is the desired formula (1.40).

\subsection{The two-dimensional case}

In the case when $N=1$ of Proposition 1.5, the first term in the expression of $\mathcal{W}(\nabla H)$ does not appear, and the lattice $\Lambda$ is such that $\mathbb{T}$ has volume 1 . Then the minimum $\mathcal{W}$-energy corresponding to the configuration $\Lambda$ can be expressed as follows, using the Fourier series expression for the function $G=G_{\Lambda}$ obtained like in the above proof:

$$
\mathcal{W}(\Lambda)=c_{d, s} \lim _{x \rightarrow 0}\left(c_{d, s} G_{\Lambda}(x)-g(x)\right)=c_{d, s} \lim _{x \rightarrow 0}\left(c_{d, s} \sum_{q \in \Lambda^{*} \backslash\{0\}} \frac{e^{2 i \pi q \cdot x}}{|2 \pi q|^{2 \alpha}}-g(x)\right),
$$

where $\Lambda^{*}$ denotes the dual lattice to $\Lambda$. In dimension $d=2$ we may use, like in [47], the number theory results from $[16,25,39,42]$ to characterize the lattice of smallest $\mathcal{W}$-energy, allowing us to prove Theorem 3. Recalling that $g(x)$ is the solution to $(-\Delta)^{\alpha} g=c_{d, s} \delta_{0}$ in $\mathbb{R}^{2}$, we have that $U_{\Lambda}:=G_{\Lambda}-c_{d, s}^{-1} g(x)$ is regular near 0 , and we have $\mathcal{W}(\Lambda)=c_{d, s}^{2} U_{\Lambda}(0)$.

Instead of the function $g(x)$ we will use a function $g_{*}(x)$ which has the following properties (where $\mathcal{F}$ denotes the Fourier transform):

$$
\left\{\begin{array}{l}
\mathcal{F} g_{*}(\xi)=\mathcal{F} g(\xi) \quad \text { outside } B_{1}, \text { and } \mathcal{F} g_{*} \text { is finite in } B_{1} \\
g(x)-g_{*}(x) \text { is } C^{\infty}
\end{array}\right.
$$

To construct $g_{*}$ it suffices to note that both $g(x)$ and $\mathcal{F} g(\xi)$ are in $L_{\text {loc }}^{1}\left(\mathbb{R}^{2} \backslash\{0\}\right)$ for our range of exponents, since $s, 2 \alpha \in(0,2)$. It follows that, for $\eta$ smooth with support 
in $B_{1}$, equal to 1 in a neighborhood of the origin, $\eta(\xi) \mathcal{F} g(\xi)$ is a compactly supported distribution; therefore, its inverse Fourier transform is $C^{\infty}$ (see [32, 2.1.3]). We then define $g_{*}(x):=g(x)-\mathcal{F}^{-1}(\eta \mathcal{F} g)(x)$, and the above properties are easily verified.

By replacing $g_{*}$ for $g$ in definition (3.4) of $\mathcal{W}$, we define the functional

$$
\mathcal{W}_{*}(\Lambda):=c_{d, s}^{2} \lim _{x \rightarrow 0}\left(G_{\Lambda}(x)-\frac{g_{*}(x)}{c_{d, s}}\right)=c_{d, s}^{2} \lim _{x \rightarrow 0}\left(\sum_{q \in \Lambda^{*} \backslash\{0\}} \frac{e^{2 i \pi q \cdot x}}{|2 \pi q|^{2 \alpha}}-\frac{g_{*}(x)}{c_{d, s}}\right) .
$$

Note that $\mathcal{W}_{*}$ and $\mathcal{W}$ differ by a constant; thus have the same minimizers, since $g(x)-$ $g_{*}(x)$ is a regular function near zero. It thus suffices to prove the result for $\mathcal{W}_{*}$.

\subsection{Minimum of the Epstein zeta function in two dimensions}

We now recall a result from [16] regarding the analytic continuation of the Epstein zeta function. Without loss of generality, we may just consider lattices of the canonical form

$$
\Lambda_{\tau}:=y^{-1 / 2}((x, y) \mathbb{Z}+(1,0) \mathbb{Z}), \quad \text { with } \tau=x+i y, y>0, x \in[0,1) .
$$

Let us denote the corresponding Epstein zeta function with exponent $2 \alpha$ by

$$
Z_{\tau}(\alpha):=\sum_{q \in \Lambda_{\tau} \backslash\{0\}} \frac{1}{|q|^{2 \alpha}}=\sum_{(m, n) \in \mathbb{Z}^{2} \backslash\{0\}} \frac{y^{\alpha}}{\left((m x+n)^{2}+m^{2} y^{2}\right)^{\alpha}} .
$$

This series converges only for $\operatorname{Re}(\alpha)>1$. We note that $Z_{\tau}$ is formally periodic in $x$ of period 1. The following formula is stated in [21], proved in [3], and obtained by manipulating the Fourier series in $x$ of $Z$ :

$$
Z_{\tau}(\alpha)=2 y^{\alpha} \zeta(2 \alpha)+2 y^{1-\alpha} \zeta(2 \alpha-1) \frac{\Gamma\left(\frac{1}{2}\right) \Gamma\left(\alpha-\frac{1}{2}\right)}{\Gamma(\alpha)}+Q(x, y, \alpha),
$$

where $\zeta(u):=\sum_{k=1}^{\infty} \frac{1}{k^{u}}$ is the Riemann zeta function,

$$
Q(x, y, \alpha):=\frac{8 \pi^{\alpha} y^{-1 / 2}}{\Gamma(\alpha)} \sum_{r=1}^{\infty} r^{\alpha-\frac{1}{2}} \sigma_{1-2 \alpha}(r) K_{\alpha-\frac{1}{2}}(2 \pi r y) \cos (2 \pi r x),
$$

and $\sigma_{\beta}(k)=\sum_{d \mid k} d^{\beta}$ and $K_{v}(z)=\int_{0}^{\infty} e^{-z \cosh t} \cosh (v t) d t$ is the so-called modified Bessel function of the second kind, which for positive real $v, z$ decays exponentially. However, if we consider the expression for $Z_{\tau}$, we see that the terms involving the Riemann zeta function again converge only for $\operatorname{Re}(\alpha)>1$, i.e. precisely outside our interest range. But we may use the functional equation

$$
\zeta(s)=2^{s} \pi^{s-1} \sin \left(\frac{\pi s}{2}\right) \Gamma(1-s) \zeta(1-s)
$$

to extend the Riemann zeta function to $\operatorname{Re}(\alpha)<1$. Formula (3.9) then gives the analytic continuation of $Z_{\tau}(\alpha)$ to a meromorphic function on all $\alpha \in \mathbb{C} \backslash\{1\}$, which has a pole of residue $\pi$ at 1 . Note that the value given by formula (3.8) coincides with the one given by (3.9) as long as it is defined, by uniqueness of the analytic continuation, and since there are no branch points. The useful point to note is that for $\left(x^{\prime}, y^{\prime}\right) \neq(x, y)$ the difference $Z_{\tau}(\alpha)-Z_{\tau^{\prime}}(\alpha)$ extends by continuity in $\alpha$ over 1 , the two poles canceling each other.

We are then able to use the following result. 
Theorem $7[16,25]$. Let $\alpha \in \mathbb{C}, \operatorname{Re}(\alpha)>0$. Under the same assumptions and using the same notation for $\tau, \Lambda_{\tau}$ as above, if $Z_{\tau}(\alpha)$ is the analytic continuation in $\alpha$ as above, then for all $x \in(0,1)$ and $y>0$ it holds that

$$
Z_{\tau}(\alpha)-Z_{e^{i \pi / 3}}(\alpha) \geqslant 0,
$$

with strict inequality for $\tau \neq e^{i \pi / 3}$.

The lattice $\Lambda_{t r i}$ corresponding to $\tau=e^{i \pi / 3}$ or $(x, y)=(1 / 2, \sqrt{3} / 2)$ is the triangular lattice.

\subsection{Relation between $\mathcal{W}_{*}$ and $Z_{\tau}$ and proof of Theorem 3}

Theorem 3 will follow from Theorem 7 once we prove the following proposition.

Proposition 3.1. Let $d=2, \alpha=\frac{d-s}{2} \in(0,1)$, and assume that $\Lambda_{\tau} \subset \mathbb{R}^{2}$ is a unit volume lattice. It holds that

$$
\mathcal{W}_{*}\left(\Lambda_{\tau}\right)-\mathcal{W}_{*}\left(\Lambda_{t r i}\right)=\frac{c_{d, s}^{2}}{(2 \pi)^{2 \alpha}}\left(Z_{\tau}(\alpha)-Z_{e^{i \pi / 3}}(\alpha)\right) .
$$

Proof. The left-hand side and right-hand side of (3.11) agree for $\alpha=\frac{d-s}{2}>1$, because in view of (3.6) the following holds:

$$
\begin{aligned}
\mathcal{W}_{*}\left(\Lambda_{\tau}\right)-\mathcal{W}_{*}\left(\Lambda_{t r i}\right) & =c_{d, s}^{2} \lim _{x \rightarrow 0}\left(\sum_{q \in \Lambda_{\tau}^{*} \backslash\{0\}} \frac{e^{2 i \pi q \cdot x}}{|2 \pi q|^{2 \alpha}}-\sum_{q \in \Lambda_{t r i}^{*} \backslash\{0\}} \frac{e^{2 i \pi q \cdot x}}{|2 \pi q|^{2 \alpha}}\right) \\
& =c_{d, s}^{2}\left(\sum_{q \in \Lambda^{*} \backslash\{0\}} \frac{1}{|2 \pi q|^{2 \alpha}}-\sum_{q \in \Lambda_{t r i}^{*} \backslash\{0\}} \frac{1}{|2 \pi q|^{2 \alpha}}\right) \\
& =\frac{c_{d, s}^{2}}{(2 \pi)^{2 \alpha}}\left(Z_{\tau}(\alpha)-Z_{e^{i \pi / 3}}(\alpha)\right) .
\end{aligned}
$$

This is justified since the above sums giving the traditional definition of $Z(\alpha)$ converge absolutely. For the same reason, the above formula for $\mathcal{W}_{*}(\Lambda)-\mathcal{W}_{*}\left(\Lambda_{\text {tri }}\right)$ can be extended to the complex half-plane $\operatorname{Re}(\alpha)>1$. We also have that the right-hand side of $(3.11)$ is analytic in $\alpha$ for $\alpha \neq 1$; thus we only need to show that the left-hand side is analytic in $\alpha$ too. To prove this we use a smoothed sum method, via a very basic Euler-Maclaurin-type error estimate valid in two dimensions.

To that aim, let us consider a positive Schwartz function $\eta: \mathbb{R}^{2} \rightarrow[0,1]$ such that $\eta=1$ on $B_{1 / 2}$ and $\eta=0$ outside $B_{1}$. Then the inverse Fourier transform $\varphi$ of $\eta$ is a Schwartz function with integral equal to 1 , and the functions $\varphi_{N}(x):=N^{2} \varphi(N x)=\left(\mathcal{F}^{-1} \eta\right)(\xi / N)$ approximate a Dirac mass at the origin. The following holds:

$$
\begin{aligned}
\lim _{x \rightarrow 0}\left(G_{\Lambda}(x)-c_{d, s}^{-1} g_{*}(x)\right) & =\lim _{N \rightarrow \infty} \int_{\mathbb{R}^{2}} \varphi_{N}(x)\left(G_{\Lambda}(x)-c_{d, s}^{-1} g_{*}(x)\right) d x \\
& =\lim _{N \rightarrow \infty} \int_{\mathbb{R}^{2}} \eta(\xi / N)\left(\mathcal{F} G_{\Lambda}(\xi)-c_{d, s}^{-1} \mathcal{F} g_{*}(\xi)\right) d \xi
\end{aligned}
$$


We may write the integral of the last line as a sum over the Voronoi cells $K_{p}:=\left\{x \in \mathbb{R}^{2}\right.$ : $\left.|x-p|=\min _{p^{\prime} \in \Lambda^{*}}\left|x-p^{\prime}\right|\right\}$ for $p \in \Lambda^{*}$, and use (3.5), to get

$$
\begin{aligned}
\int_{\mathbb{R}^{2}} & \eta(\xi / N)\left(\mathcal{F} G_{\Lambda}(\xi)-c_{d, s}^{-1} \mathcal{F} g_{*}(\xi)\right) d \xi \\
= & \sum_{p \in\left(\Lambda^{*} \backslash\{0\}\right) \cap B_{1}} \frac{\eta(p / N)}{|2 \pi p|^{2 \alpha}}-c_{d, s}^{-1} \sum_{p \in \Lambda^{*} \cap B_{1}} \int_{K_{p}} \eta(\xi / N) \mathcal{F} g_{*}(\xi) d \xi \\
& +\sum_{p \in \Lambda^{*} \backslash B_{1}}\left(\frac{\eta(p / N)}{|2 \pi p|^{2 \alpha}}-\int_{K_{p}} \frac{\eta(\xi / N)}{|2 \pi \xi|^{2 \alpha}} d \xi\right) \\
:= & I_{1}(\Lambda, \alpha)+I_{2}(\Lambda, \alpha) .
\end{aligned}
$$

We note that the term $I_{1}(\Lambda, \alpha)$ is just the sum of a finite (depending on $\Lambda=\Lambda_{\tau}^{*}$ but locally bounded with respect to $\tau$ ) number of terms; in particular it is analytic in $\alpha$ and converges uniformly in $N$ for $\tau$ in a fixed compact set and $\alpha$ in a fixed compact subset within $\operatorname{Re}(\alpha)>0$. Therefore it suffices to focus on the term $I_{2}(\Lambda, \alpha)$. The terms in the sum defining it are of the form

$$
M_{p}^{N}:=\int_{K_{p}}\left(\frac{\eta(p / N)}{|2 \pi p|^{2 \alpha}}-\frac{\eta(\xi / N)}{|2 \pi \xi|^{2 \alpha}}\right) d \xi, p \in \Lambda^{*} \backslash B_{1} .
$$

For real $\alpha>0$, define $f_{N}(\xi):=|2 \pi \xi|^{-2 \alpha} \eta(\xi / N), \varphi_{N}=f_{N}-f_{N / 2}$, which by choice of $\eta$ is supported in $B_{N} \backslash B_{N / 4}$. Its Taylor expansion is

$$
\varphi_{N}(p)-\varphi_{N}(\xi)+D \varphi_{N}(p) \cdot(\xi-p)=E_{N}(\xi)
$$

with

$$
\left\|E_{N}\right\|_{L^{\infty}\left(K_{p}\right)} \leqslant C\left\|D^{2} \varphi_{N}\right\|_{L^{\infty}\left(K_{p}\right)} \max _{\xi \in K_{p}}|\xi-p|^{2}=C_{\Lambda}\left\|D^{2} \varphi_{N}\right\|_{L^{\infty}\left(K_{p}\right)},
$$

and by a direct computation we have

$$
\left\|D^{2} \varphi_{N}\right\|_{L^{\infty}\left(K_{p}\right)} \leqslant C_{\eta, \alpha, \Lambda}\left(\frac{1}{N^{2}|p|^{2 \alpha}}+\frac{1}{N|p|^{2 \alpha+1}}+\frac{1}{|p|^{2 \alpha+2}}\right) .
$$

Choosing now $N=2^{h}$, we may write $f_{N}=f_{2^{k_{\Lambda}}}+\sum_{k=k_{\Lambda}+1}^{h} f_{2^{k}}-f_{2^{k-1}}=f_{2^{k_{\Lambda}}}+$ $\sum_{k=k_{\Lambda}+1}^{h} \varphi_{2^{k}}$, and thus

$$
\left|M_{p}^{N}\right| \leqslant \int_{K_{p}}\left|f_{2^{k_{\Lambda}}}(\xi)-f_{2^{k_{\Lambda}}}(p)\right|+C_{\Lambda} \sum_{k=k_{\Lambda}+1}^{h} \int_{K_{p}}\left|E_{2^{k}}(\xi)\right| d \xi,
$$

where we used the fact that, since the domain $K_{p}$ is symmetric with respect to $p$, the integral of the first order term in the Taylor expansion is zero. We now sum the bounds (3.12)-(3.13) for the contributions (3.14) over $p \in \Lambda^{*}$ such that $K_{p}$ intersects $B_{2^{k}} \backslash B_{2^{k-2}}$. In this case $2^{k-2}-C_{\Lambda} \leqslant|p| \leqslant 2^{k}+C_{\Lambda}$, and, since each $K_{p}$ has volume 1 , the number of such $p$ is $\leqslant\left(2^{k}+C_{\Lambda}\right)^{2}$. Choosing $k_{\Lambda}$ minimal such that $2^{k_{\Lambda}} \geqslant 8 C_{\Lambda}$, we have

$$
\sum_{p \in \Lambda^{*} \backslash B_{1}}\left|M_{p}^{2^{h}}\right| \leqslant C_{\alpha, \eta, \Lambda}+C_{\Lambda} \sum_{k=k_{\Lambda}}^{h} \sum_{2^{k-2}-C_{\Lambda} \leqslant|p| \leqslant 2^{k}+C_{\Lambda}}\left\|D^{2} \varphi_{2^{k}}\right\|_{L^{\infty}\left(K_{p}\right)}
$$




$$
\begin{aligned}
& \leqslant C_{\alpha, \eta, \Lambda}+C_{\Lambda} \sum_{k=k_{\Lambda}}^{h}\left(2^{k}+C_{\Lambda}\right)^{2} \frac{1}{\left(2^{k}\right)^{2+2 \alpha}} \\
& \leqslant C_{\alpha, \eta, \Lambda}+C_{\Lambda} \sum_{k=k_{\Lambda}}^{\infty} 2^{2(k+1)-(2+2 \alpha) k} \\
& =C_{\alpha, \eta, \Lambda}+2^{-2 k_{\Lambda} \alpha} C_{\Lambda, \alpha}^{\prime} .
\end{aligned}
$$

In the last estimate we used the fact that $\alpha>0$; thus the series $\sum_{k=1}^{\infty} 2^{-2 k \alpha}$ converges. The same reasoning easily extends to the case when $\operatorname{Re}(\alpha)>0$.

We thus see that the series in $M_{p}^{N}$ converges absolutely and uniformly in $N$ for $\tau, \alpha$ inside a fixed compact set respecting the given constraints. Since $\eta$ had bounded support, just finitely many terms contribute to each sum, and by Morera's theorem the series gives a function which is analytic in $\alpha$. By local uniformity of the convergence, the limit in $N$ remains analytic in $\alpha$ for $\operatorname{Re}(\alpha)>0$.

As discussed at the beginning of the proof, the left-hand side and the right-hand side of (3.10) agree for $\operatorname{Re}(\alpha)>1$, while they are both analytic on $\operatorname{Re}(\alpha)>0$, so by analytic continuation they must also agree on $\operatorname{Re}(\alpha)>0$. This completes the proof.

\section{Separation of points}

The proofs in this section follow [43, 44], which themselves are inspired by [37].

The first lemma expresses that, if a configuration minimizes $H_{n}$, each point must be a minimum for the potential generated by the rest.

Lemma 4.1. Let $x_{1}, \ldots, x_{n} \in \mathbb{R}^{d}$ minimize $H_{n}$. Let $x_{i}^{\prime}$ be the blown-up points and $h_{n}^{\prime}$ be associated as in (1.44), and let $U(X)=h_{n}^{\prime}(X)-g\left(X-x_{1}^{\prime}\right)$. Then, for any $\bar{x} \in \mathbb{R}^{d}$ and $\bar{x}^{\prime}=n^{1 / d} \bar{x}$, we have

$$
n^{s / d} U\left(x_{1}^{\prime}\right)+2 n \zeta\left(x_{1}\right) \leqslant n^{s / d} U\left(\bar{x}^{\prime}\right)+2 n \zeta(\bar{x}) .
$$

Proof. Let us denote $\tilde{h}_{n}=g *\left(\sum_{i=2}^{n} \delta_{x_{i}^{\prime}}+\delta_{\bar{x}^{\prime}}-\mu_{V}^{\prime} \delta_{\mathbb{R}^{d}}\right)$. We note that $h_{n}$ and $\tilde{h}_{n}$ correspond to the potential generated by a zero total charge, so they both decay like $|x|^{-s-1}$ as $x \rightarrow \infty$, while their gradients decay like $|x|^{-s-2}$.

We also let $\bar{h}(X)=\tilde{h}_{n}(X)-h_{n}(X)=g\left(X-\bar{x}^{\prime}\right)-g\left(X-x_{1}^{\prime}\right)$, and note that it decreases in the same way at infinity.

Expanding the square, we write

$$
\int_{\mathbb{R}^{d+k}}|y|^{\gamma}\left|\nabla \tilde{h}_{n, \eta}\right|^{2}=\int_{\mathbb{R}^{d+k}}|y|^{\gamma}\left|\nabla h_{n, \eta}\right|^{2}+\int_{\mathbb{R}^{d+k}}|y|^{\gamma}\left|\nabla \bar{h}_{\eta}\right|^{2}+2 \int_{\mathbb{R}^{d+k}}|y|^{\gamma} \nabla h_{n, \eta} \cdot \nabla \bar{h}_{\eta} .
$$

Using Green's theorem and the decay of $\bar{h}$ and $\nabla \bar{h}$ at infinity, we have, if $\bar{x}^{\prime} \neq x_{1}^{\prime}$,

$$
\begin{aligned}
\int_{\mathbb{R}^{d+k}}|y|^{\gamma}\left|\nabla \bar{h}_{\eta}\right|^{2} & =c_{d, s} \int_{\mathbb{R}^{d+k}}\left(\delta_{\bar{x}^{\prime}}^{(\eta)}-\delta_{x_{1}^{\prime}}^{(\eta)}\right)\left(g\left(X-\bar{x}^{\prime}\right)-g\left(X-x_{1}^{\prime}\right)\right) \\
& =2 c_{d, s} g(\eta)-2 c_{d, s} g\left(\bar{x}^{\prime}-x_{1}^{\prime}\right)+o(1)
\end{aligned}
$$


as $\eta \rightarrow 0$. On the other hand, still if $\bar{x}^{\prime} \neq x_{1}^{\prime}$,

$$
\begin{aligned}
\int_{\mathbb{R}^{d+k}}|y|^{\gamma} \nabla h_{n, \eta} \cdot \nabla \bar{h}_{\eta}= & c_{d, s} \int\left(g\left(X-\bar{x}^{\prime}\right)-g\left(X-x_{1}^{\prime}\right)\right)\left(\sum_{i=1}^{n} \delta_{x_{i}^{\prime}}^{(\eta)}-\mu_{V}^{\prime} \delta_{\mathbb{R}^{d}}\right) \\
= & c_{d, s}\left(\sum_{i=2}^{n} g\left(x_{i}^{\prime}-\bar{x}^{\prime}\right)-g\left(x_{i}^{\prime}-x_{1}^{\prime}\right)-g(\eta)+g\left(x_{1}^{\prime}-\bar{x}^{\prime}\right)\right) \\
& -c_{d, s} \int\left(g\left(X-\bar{x}^{\prime}\right)-g\left(X-x_{1}^{\prime}\right)\right) \mu_{V}^{\prime} \delta_{\mathbb{R}^{d}}+o(1)
\end{aligned}
$$

as $\eta \rightarrow 0$. We deduce that, if $\bar{x}^{\prime} \neq x_{1}^{\prime}$,

$$
\lim _{\eta \rightarrow 0} \int_{\mathbb{R}^{d+k}}|y|^{\gamma}\left|\nabla \tilde{h}_{n, \eta}\right|^{2}-\int_{\mathbb{R}^{d+k}}|y|^{\gamma}\left|\nabla h_{n, \eta}\right|^{2}=U\left(\bar{x}^{\prime}\right)-U\left(x_{1}^{\prime}\right),
$$

and of course there is equality as well if $\bar{x}^{\prime}=x_{1}^{\prime}$. Combining with the splitting formula, Proposition 1.6, it follows that (4.1) holds for minimizers.

We now state a maximum principle proven in [27, Theorem 2.2.2] for operators with weights in the Muckenhoupt $A_{2}$ class, which contain the operator $-\operatorname{div}\left(|y|^{\gamma} \nabla \cdot\right)$ that we are using.

Lemma 4.2. Assume that $h$ satisfies

$$
-\operatorname{div}\left(|y|^{\gamma} \nabla h\right) \geqslant 0
$$

in some open subset $U$ of $\mathbb{R}^{d+k}$, and $\int_{U}|y|^{\gamma}|\nabla h|^{2}<+\infty$. Then $h$ does not have any local minimum in $U$.

We are now in a position to deduce the following.

Proof of Theorem 5. First we prove that all the points are in $\Sigma \times\{0\}$. Take a minimizing configuration, blow it up, and let $U$ be as in Lemma 4.1. Let also $\Sigma^{\prime}$ be $n^{1 / d} \Sigma$, the blown-up of the equilibrium measure support. Since $h_{n}^{\prime}$ satisfies (1.46), we see that $U$ satisfies $-\operatorname{div}\left(|y|^{\gamma} \nabla U\right)=g * \mu$, where $\mu$ is a measure of total mass $-c_{d, s}$ and is compactly supported in $\mathbb{R}^{d} \times\{0\}$. Thus $U$ must be asymptotic to $-g$ at infinity. In cases (1.3)-(1.4), this implies that $U \rightarrow+\infty$ as $|X| \rightarrow+\infty$, and in case (1.2), this implies that $U(X)<0$ for $|X|$ large enough, while $U(X) \rightarrow 0$ as $|X| \rightarrow+\infty$. In addition, $U(X) \rightarrow+\infty$ as $X \rightarrow x_{i}^{\prime}$ for some $i \neq 1$. In both cases, it follows that $U$ must achieve a minimum somewhere in $\mathbb{R}^{d+k} \backslash \bigcup_{i=2}^{n}\left\{x_{i}^{\prime}\right\}$. But, from (1.46), we have that $-\operatorname{div}\left(|y|^{\gamma} \nabla U\right) \geqslant 0$ in $\mathbb{R}^{d+k} \backslash\left(\Sigma^{\prime} \times\{0\} \cup\right.$ $\left.\bigcup_{i=2}^{n}\left\{x_{i}^{\prime}\right\}\right)$, which is an open set. Also $|y|^{\gamma}|\nabla U|^{2}$ is locally integrable away from the points $x_{i}^{\prime}$. The maximum principle, Lemma 4.2, thus applies to $U$ away from the points $x_{i}^{\prime}$, and implies that the minimum must be in $\Sigma^{\prime} \times\{0\}$ (because it cannot be at any $x_{i}^{\prime}$ for $i \geqslant 2$ ). Call $\bar{x}^{\prime}$ this point, and let $\bar{x}=n^{-1 / d} \bar{x}^{\prime}$. In view of Lemma 4.1 , since $\zeta(\bar{x})=0$, we have

$$
U\left(\bar{x}^{\prime}\right) \leqslant U\left(x_{1}^{\prime}\right) \leqslant U\left(\bar{x}^{\prime}\right)-2 n^{1-s / d} \zeta\left(x_{1}\right),
$$

which implies (since $\zeta \geqslant 0$ ) that $U\left(x_{1}^{\prime}\right)=U\left(\bar{x}^{\prime}\right)$, and thus $x_{1}^{\prime}$ is also a point of minimum of $U$. Thus $x_{1}^{\prime} \in \Sigma^{\prime}$ by the maximum principle. Since the system is invariant under relabeling, this shows that all the points $x_{i}$ are in $\Sigma$, as claimed. 
We next prove the separation result. Now that we know that all the points are in $\Sigma$, (4.1) gives that, for any $\bar{x}^{\prime} \in \mathbb{R}^{d}$,

$$
U\left(x_{1}^{\prime}\right) \leqslant U\left(\bar{x}^{\prime}\right)+2 n^{1-s / d} \zeta(\bar{x}) .
$$

Let $\rho=\left(\frac{1}{\omega_{d} \bar{m}}\right)^{\frac{1}{d}}$, where $\omega_{d}$ is the volume of the unit ball in dimension $d$, and $\bar{m}$ is the constant in (1.14). Let $x_{2}^{\prime}$ be some point in the collection, and let us assume that $x_{1}^{\prime} \in B\left(x_{2}^{\prime}, \rho\right)$ (up to relabeling). Note that the choice of $\rho$ ensures that $\bar{m} \mathcal{H}^{d}\left(B\left(x_{2}^{\prime}, \rho\right) \cap\right.$ $\left.\left.\mathbb{R}^{d} \times\{0\}\right)\right)<1$. We next split $U$ into

$$
U=U^{n e a r}+U^{r e m}
$$

where

$$
\begin{gathered}
U^{\text {near }}=g *\left(\delta_{x_{2}^{\prime}}-\bar{m} \mathbb{1}_{B\left(x_{2}^{\prime}, \rho\right)} \delta_{\mathbb{R}^{d}}\right) \\
U^{\text {rem }}=g *\left(\sum_{i=3}^{n} \delta_{x_{i}^{\prime}}-\mu_{V}^{\prime} \delta_{\mathbb{R}^{d}}+\bar{m} \mathbb{1}_{B\left(x_{2}^{\prime}, \rho\right)} \delta_{\mathbb{R}^{d}}\right) .
\end{gathered}
$$

One may observe that

$$
-\operatorname{div}\left(|y|^{\gamma} \nabla U^{r e m}\right) \geqslant c_{d, s}\left(\bar{m} \mathbb{1}_{B\left(x_{2}^{\prime}, \rho\right)}-\mu_{V}^{\prime}\right) \delta_{\mathbb{R}^{d}} \geqslant 0 \quad \text { in }\left(\Sigma^{\prime}\right)^{c} \cup B\left(x_{2}^{\prime}, \rho\right)
$$

because we always have $\bar{m} \geqslant \mu_{V}^{\prime}$. Moreover, since $-\int \operatorname{div}\left(|y|^{\gamma} \nabla U^{r e m}\right)=c_{d, s}\left(-3+\bar{m} \omega_{d} \rho^{d}\right)$ is a negative number $C \leqslant-2 c_{d, s}$ by choice of $\rho, U^{\text {rem }}$ is asymptotic to $\frac{C}{c_{d, s}} g$ at infinity. By the same reasoning as above it follows that $U^{\text {rem }}$ achieves a minimum somewhere. By Lemma 4.2 this minimum is at some point $\bar{x}^{\prime}$ which cannot be in $\left(\Sigma^{\prime}\right)^{c} \cup B\left(x_{2}^{\prime}, \rho\right)$, and hence is in the closure of $\left(\Sigma^{\prime} \backslash B\left(x_{2}^{\prime}, \rho\right)\right) \times\{0\}$. Thus

$$
U^{r e m}\left(\bar{x}^{\prime}\right) \leqslant U^{r e m}\left(x_{1}^{\prime}\right) \quad \zeta(\bar{x})=0 .
$$

We next turn to $U^{\text {near }}$ and show that, if $|x| \bar{m}^{1 / d}$ is smaller than some constant $r>0$ depending only on $d$ and $s$, then

$$
U^{\text {near }}(x) \geqslant \max _{B\left(x_{2}^{\prime}, \rho\right)^{c}} U^{\text {near }} .
$$

By change of origin, we may assume that $x_{2}^{\prime}=0$. By scaling, it also suffices to prove this for $\bar{m}=1$ and $\rho=\omega_{d}^{-1 / d}$. We note that then $U^{\text {near }} \leqslant g+C$, where the constant depends only on $s$ and $d$, and can be taken to be 0 in the Riesz cases (it is there to account for the possible negativity of $-\log |x|$ in the logarithmic case), so that $\max _{B(0, \rho)^{c}} U^{\text {near }} \leqslant$ $g(\rho)+C=g\left(\omega_{d}^{-1 / d}\right)+C$. On the other hand, in the Riesz case,

$$
\begin{aligned}
U^{\text {near }}(x) & =g(x)-\int_{y \in B(0, \rho)} g(y-x) d y \\
& \geqslant g(x)-\int_{y \in B(0,|x|+\rho)} g(y) d y \geqslant g(x)-C(\rho+|x|)^{d-s},
\end{aligned}
$$

and the conclusion follows immediately. One easily checks that the conclusion holds as well in the logarithmic cases, and one can also note that an explicit estimate of the best $r$ is possible. 
If $r$ is taken as above, then, if $x_{1}^{\prime} \in B\left(x_{2}^{\prime}, r \bar{m}^{-1, d}\right)$, this implies from (4.6) that $U^{\text {near }}\left(\bar{x}^{\prime}\right)<$ $U^{\text {near }}\left(x_{1}^{\prime}\right)$. Combining this with (4.5) and (4.4), it follows that $U\left(\bar{x}^{\prime}\right)<U\left(x_{1}^{\prime}\right)$ and $\zeta(\bar{x})=0$, a contradiction with (4.3). Thus, for any pair of points $x_{1}^{\prime}, x_{2}^{\prime}$ (up to relabeling), we must have $\left|x_{1}^{\prime}-x_{2}^{\prime}\right| \geqslant r \bar{m}^{-1 / d}$. Scaling down concludes the proof.

Proposition 4.3. Assume that $E$ is a minimizer of $\mathcal{W}$ over the class of vector fields in $\mathcal{A}_{1}$ which are $R$-periodic, for some given $R$ such that $\left|K_{R}\right| \in \mathbb{N}$. Then, letting $\Lambda$ be the associated set of points, we have

$$
\min _{p, q \in \Lambda, p \neq q}|p-q| \geqslant r
$$

where $r$ is a positive constant depending only on $s$ and $d$ (the same as in Theorem 5).

Note that such a minimizer exists by the explicit formula in Proposition 1.5.

Proof. The argument is the same as above. First, we note that the points of $E$ must have single multiplicities, otherwise $\mathcal{W}(E)$ would be infinite by Proposition 1.5. Second, we let $h$ be the periodic potential generated by the $N=\left|K_{R}\right|$ minimizing points $x_{1}, \ldots, x_{N}$, and $G$ be the periodic Green function of the operator $-\operatorname{div}\left(|y|^{\gamma} \nabla \cdot\right)$, as in $(3.2)$. Let $U(X)=h(X)-G\left(X-x_{1}\right)$, and let $\tilde{h}=G *\left(\sum_{i=2}^{N} \delta_{x_{i}}+\delta_{\bar{x}}-\delta_{\mathbb{R}^{d}}\right)$ and $\bar{h}(X)=\tilde{h}(X)-h(X)=G(X-\bar{x})-G\left(X-x_{1}\right)$. Computing exactly as in Lemma 4.1, we find that $\mathcal{W}(\nabla \tilde{h})-\mathcal{W}(\nabla h)=U(\bar{x})-U\left(x_{1}^{\prime}\right)$; hence, since $\left(x_{1}, \ldots, x_{N}\right)$ minimize $\mathcal{W}$, we must have that $x_{1}^{\prime}$ is a minimum of $U$.

Next, we assume by contradiction that there is a point in the collection, say $x_{2}$, such that $x_{1} \in B\left(x_{2}, r\right)$, where $r$ is as in Theorem 5 , and write

$$
U=U^{\text {near }}+U^{r e m}
$$

where

$$
\begin{gathered}
U^{\text {near }}=G *\left(\delta_{x_{2}}-\mathbb{1}_{B\left(x_{2}, \rho\right)} \delta_{\mathbb{R}^{d}}\right) \\
U^{r e m}=G *\left(\sum_{i \neq 1,2} \delta_{x_{i}}+\left(\mathbb{1}_{B\left(x_{2}, \rho\right)}-1\right) \delta_{\mathbb{R}^{d}}\right) .
\end{gathered}
$$

By the definition of $G$, we have

$$
\begin{aligned}
-\operatorname{div}\left(|y|^{\gamma} \nabla U^{r e m}\right) & =c_{d, s}\left(\sum_{i \neq 1,2} \delta_{x_{i}}+\left(\mathbb{1}_{B\left(x_{2}, \rho\right)}-1-\frac{-2+\left|B\left(x_{2}, \rho\right)\right|}{N}\right) \delta_{\mathbb{R}^{d}}\right) \\
& \geqslant c_{d, s}\left(\left(\mathbb{1}_{B\left(x_{2}, \rho\right)}-1\right) \delta_{\mathbb{R}^{d}}\right),
\end{aligned}
$$

where we used that $\left|B\left(x_{2}, \rho\right)\right|<1$, i.e. $-\operatorname{div}\left(|y|^{\gamma} \nabla U^{r e m}\right) \geqslant 0$ in $B\left(x_{2}, \rho\right) \cup\left(\mathbb{R}^{d}\right)^{c}$. Moreover, since $G$ has average 0 in each periodicity cell of $\mathbb{R}^{d}, U^{\text {rem }}$ too, so $U^{\text {rem }}$ takes negative values unless it is constant. But $U^{r e m}$ is periodic in the $x \in \mathbb{R}^{d}$ direction and tends to 0 as $|y| \rightarrow \infty$ (like $G$ ), so $U^{\text {rem }}$ must achieve a minimum at some point. By Lemma 4.2, this minimum $\bar{x}$ can only be in $\left(\mathbb{R}^{d} \backslash B\left(x_{2}, \rho\right)\right) \times\{0\}$. On the other hand, we saw in the proof of Theorem 5 that $U^{\text {near }}\left(x_{1}\right)>\max _{B\left(x_{2}, \rho\right)^{c}} U^{\text {near }}$. It follows that if, $\left|x_{1}-x_{2}\right|<r$, we have $U(\bar{x})<U\left(x_{1}\right)$, a contradiction. 


\section{Lower bound with the ergodic theorem approach}

In this section, we turn to obtaining a lower bound for the energy of arbitrary (nonnecessarily minimizing) configurations. From Proposition 1.6 and Lemma 2.3 we already have the following: for all $\eta<1$,

$$
\begin{aligned}
& H_{n}\left(x_{1}, \ldots, x_{n}\right)-n^{2} \mathcal{E}\left(\mu_{V}\right) \\
& \quad \geqslant 2 n \sum_{i=1}^{n} \zeta\left(x_{i}\right)+n^{1+\frac{s}{d}}\left(\frac{1}{n} \int_{\mathbb{R}^{d+k}}|y|^{\gamma}\left|\nabla h_{n, \eta}^{\prime}\right|^{2}-c_{d, s} g(\eta)-C \eta^{\frac{d-s}{2}}\right),
\end{aligned}
$$

respectively in the cases (1.3)-(1.4),

$$
\begin{aligned}
& H_{n}\left(x_{1}, \ldots, x_{n}\right)-n^{2} \mathcal{E}\left(\mu_{V}\right)+\frac{n}{d} \log n \\
& \quad \geqslant 2 n \sum_{i=1}^{n} \zeta\left(x_{i}\right)+n\left(\frac{1}{n} \int_{\mathbb{R}^{d+k}}|y|^{\gamma}\left|\nabla h_{n, \eta}^{\prime}\right|^{2}-c_{d, s} g(\eta)-C \eta^{d / 2}\right),
\end{aligned}
$$

where $C$ depends only on $d, s$, and $V$. We recall that from Lemma 2.3 there is equality if $\min _{i \neq j}\left|x_{i}-x_{j}\right|>2 \eta$.

In this section we will take the limits $n \rightarrow \infty$ and $\eta \rightarrow 0$ in the above relations to provide the lower bound for the energy. This is done as in [44] according to the method for 'lower bounds for 2-scale energies' initiated in [47, 48] and inspired by Varadhan. The idea is to rewrite the energy as an average of energies computed on finite size balls after blow-up. More precisely, consider a radial smooth probability density $\chi: \mathbb{R}^{d} \rightarrow \mathbb{R}^{+}$ supported in the unit ball of $\mathbb{R}^{d}$. We may rewrite the energy of $h_{n, \eta}^{\prime}$ (which is the function defined using (1.30) and (1.44)) as

$$
\begin{aligned}
\int_{\mathbb{R}^{d+k}}|y|^{\gamma}\left|\nabla h_{n, \eta}^{\prime}\right|^{2} & =\int_{\mathbb{R}^{d+k}}\left(\int_{\mathbb{R}^{d}} \chi(x-\tilde{x}) d \tilde{x}\right)|y|^{\gamma}\left|\nabla h_{n, \eta}^{\prime}(X)\right|^{2} d X \\
& =\int_{\mathbb{R}^{d+k}} \int_{\mathbb{R}^{d}} \chi(\tilde{x})|y|^{\gamma}\left|\nabla h_{n, \eta}^{\prime}(X+(\tilde{x}, 0))\right|^{2} d \tilde{x} d X \\
& \geqslant \int_{n^{1 / d} \Sigma} \int_{\mathbb{R}^{k}} \int_{\mathbb{R}^{d}} \chi(\tilde{x})|y|^{\gamma}\left|\nabla h_{n, \eta}^{\prime}(X+(\tilde{x}, 0))\right|^{2} d \tilde{x} d y d x,
\end{aligned}
$$

where we discarded the integral over the complement of $n^{1 / d} \Sigma$ which we guessed to be unimportant. Changing variables, we obtain

$$
\int_{\mathbb{R}^{d+k}}|y|^{\gamma}\left|\nabla h_{n, \eta}^{\prime}\right|^{2} \geqslant n|\Sigma| \int_{\Sigma} \int_{\mathbb{R}^{d+k}} \chi(\tilde{x})|y|^{\gamma}\left|\nabla h_{n, \eta}^{\prime}\left(x n^{1 / d}+\tilde{x}, y\right)\right|^{2} d \tilde{x} d y d x .
$$

The method then consists in examining the local energies thus defined, i.e.

$$
\int_{\mathbb{R}^{d+k}} \chi(\tilde{x})|y|^{\gamma}\left|\nabla h_{n, \eta}^{\prime}\left(x n^{1 / d}+\tilde{x}, y\right)\right|^{2} d \tilde{x} d y,
$$

which have natural limits, and in rewriting (5.3) as an average over $x \in \Sigma$ of these energies. 
More precisely, given some configuration of points and $h_{n, \eta}^{\prime}$ its associated truncated blown-up potential, the local energy is defined as follows based on $(5.3)$, for $(x, \mathcal{Y}) \in \mathcal{X}$ ( $\mathcal{X}$ is the space $\Sigma \times L_{\text {loc }}^{p}\left(\mathbb{R}^{d+k}, \mathbb{R}^{d+k}\right.$ ) as specified in the introduction):

$$
\mathbf{f}_{n, \eta}(x, \mathcal{Y}):= \begin{cases}\int_{\mathbb{R}^{d+k}} \chi|y|^{\gamma}|\mathcal{Y}|^{2} & \text { if } \mathcal{Y}(X)=\nabla h_{n, \eta}^{\prime}\left(X+\left(x n^{1 / d}, 0\right)\right), \\ +\infty & \text { else, }\end{cases}
$$

where $\theta_{\lambda}, \lambda \in \mathbb{R}^{d}$ is the group of translations of $\mathbb{R}^{d}$, which acts on $\mathcal{X}$ by

$$
\theta_{\lambda} \mathcal{Y}(X):=\mathcal{Y}(X+(\lambda, 0))
$$

To separate scales we also consider scale- $n^{1 / d}$ coupled actions of the $\mathbb{R}^{d}$-translations on $\mathbb{R}^{d} \times \mathcal{X}$ defined as follows:

$$
T_{\lambda}^{n}(x, \mathcal{Y}):=\left(x+n^{1 / d} \lambda, \theta_{\lambda} \mathcal{Y}\right) .
$$

The global energy is defined as an average of the local ones by

$$
\mathbf{F}_{n, \eta}(\mathcal{Y}):=f_{\Sigma} \mathbf{f}_{n, \eta}\left(x, \theta_{x n^{1 / d}} \mathcal{Y}\right) d x,
$$

and (5.3) translates into the upper bound

$$
\mathbf{F}_{n, \eta}(\mathcal{Y}) \leqslant \frac{1}{|\Sigma| n} \int_{\mathbb{R}^{d+k}}|y|^{\gamma}\left|\nabla h_{n, \eta}^{\prime}\right|^{2},
$$

if $\mathbf{F}_{n, \eta}(\mathcal{Y}) \neq+\infty$. In view of (5.4) and (5.1), to bound the energy from below, it suffices to bound from below $\mathbf{F}_{n, \eta}$. Theorem 7 in [48] is precisely designed to obtain lower bounds on such energies from input at the microscopic scale (i.e. on $\mathbf{f}_{n}$ ). The idea is that $\mathbf{F}_{n, \eta}$ is roughly $\int \mathbf{f}_{n, \eta}(x, \mathcal{Y}) d P_{n, \eta}(x, \mathcal{Y})$, where $P_{n, \eta}$ is the push-forward of $P_{v_{n}}$, as defined in (1.52) by the map $\Phi_{\eta}$ (defined just after (1.30) as the one that maps $E$ to $E_{\eta}$ ), or in other words the push-forward of the normalized Lebesgue measure on $\Sigma$ by

$$
x \mapsto\left(x, \nabla h_{n, \eta}^{\prime}\left(n^{1 / d}(x, 0)+\cdot\right)\right) .
$$

Then it suffices to obtain some tightness for $\left\{P_{n, \eta}\right\}$ and pass to the limit in this average $\int \mathbf{f}_{n} d P_{n, \eta}$ to obtain a lower bound by $\int \mathbf{f}_{\eta} d P_{\eta}$, where $\mathbf{f}_{\eta}$ is identified as the liminf of $\mathbf{f}_{n, \eta}$. More precisely, if $\left(\tilde{x}_{n}, \mathcal{Y}_{n}\right) \rightarrow(\tilde{x}, \mathcal{Y})$ in $\mathcal{X}$, we have

$$
\liminf _{n \rightarrow \infty} \mathbf{f}_{n, \eta}\left(x_{n}, \mathcal{Y}_{n}\right) \geqslant \mathbf{f}_{\eta}(\tilde{x}, \mathcal{Y})
$$

where

$$
\mathbf{f}_{\eta}(\tilde{x}, \mathcal{Y}):= \begin{cases}\int_{\mathbb{R}^{d}} \chi|y|^{\gamma}|\mathcal{Y}|^{2} & \text { if } \tilde{x} \in \Sigma \text { is a point of continuity of } \mu_{V} \\ 0 & \text { and } \mathcal{Y}=\Phi_{\eta}(E) \text { for some } E \in \mathcal{A}_{\mu_{V}(\tilde{x})} \\ +\infty & \text { if } \tilde{x} \text { is a point of discontinuity of } \mu_{V} \\ \text { otherwise. }\end{cases}
$$

Showing that the limit $P=\Phi_{\eta}^{-1}\left(P_{\eta}\right)$ is admissible will ensure in particular that it is translation invariant, and that we may apply the multiparameter ergodic theorem to conclude with the desired lower bound.

The first step is to obtain the tightness of $\left\{P_{n, \eta}\right\}_{n}$. This will be obtained from the following compactness result for the local energies, analogous to [44, Lemma 4.2]. 
Lemma 5.1. Assume that $\mu_{V}$ has compact support $\Sigma$ and a density which is bounded continuous on $\Sigma$. Let $h_{n, \eta}^{\prime}$ be the truncated blown-up potential generated by a configuration of points, and let $v_{n}^{\prime}=\sum_{i=1}^{n} \delta_{x_{i}^{\prime}}$. Assume that, for every $R>1$ and for some $\eta \in(0,1)$, we have

$$
\sup _{n} \int_{B_{R} \times \mathbb{R}^{k}}|y|^{\gamma}\left|\nabla h_{n, \eta}^{\prime}\left(\left(n^{1 / d} \tilde{x}_{n}, 0\right)+\cdot\right)\right|^{2} \leqslant C_{\eta, R},
$$

and that the centering point $\tilde{x}_{n} \rightarrow \tilde{x} \in \mathbb{R}^{d}$ as $n \rightarrow \infty$. Then $\left\{v_{n}^{\prime}\left(\left(n^{1 / d} \tilde{x}_{n}, 0\right)+\cdot\right)\right\}_{n}$ is locally bounded, and up to extraction converges weakly as $n \rightarrow \infty$, in the sense of measures, to

$$
v=\sum_{p \in \Lambda} N_{p} \delta_{p}
$$

where $\Lambda$ is a discrete set and $N_{p} \in \mathbb{N}^{*}$. In addition, there exist $E, E_{\eta} \in L_{\text {loc }}^{p}\left(\mathbb{R}^{d+k}\right)$ for $1<p<\min \left(2, \frac{2}{\gamma+1}, \frac{d+k}{s+1}\right)$, with $E_{\eta}=\Phi_{\eta}(E)$ such that, up to further extraction of a subsequence,

$$
\nabla h_{n}^{\prime}\left(\left(n^{1 / d} x_{n}, 0\right)+\cdot\right) \rightarrow E \text { weakly in } L_{\mathrm{loc}}^{p} \text { as } n \rightarrow \infty
$$

and

$$
\nabla h_{n, \eta}^{\prime}\left(\left(n^{1 / d} x_{n}, 0\right)+\cdot\right) \rightarrow E_{\eta} \text { weakly in } L_{\mathrm{loc}}^{p} \text { and } L_{\left.|y|\right|^{\gamma}, \mathrm{loc}}^{2} \text { as } n \rightarrow \infty .
$$

Moreover, $E$ is a gradient, and, if $\tilde{x} \in \Sigma$ is a point of continuity of $\mu_{V}$, we have

$$
-\operatorname{div}\left(|y|^{\gamma} E\right)=c_{d, s}\left(v-\mu_{V}(\tilde{x})\right) \quad \text { in } \mathbb{R}^{d+k}
$$

hence $E \in \mathcal{A}_{\mu_{V}(\tilde{x})}$.

Proof. By a mean-value argument, we deduce from the bound (5.5) that there exists $t \in[R-1, R]$ such that

$$
\int_{\partial\left(B_{t} \times[-t, t]^{k}\right)}|y|^{\gamma}\left|\nabla h_{n, \eta}^{\prime}\left(\left(n^{1 / d} \tilde{x}_{n}, 0\right)+X\right)\right|^{2} \leqslant C_{\eta, R} .
$$

This is true for $k=1$, and for $k=0$ the integral should be understood as on $\partial B_{t}$. On the other hand, integrating (1.48) over $B\left(n^{1 / d} \tilde{x}_{n}, t\right)$ and changing variables, we have

$$
\begin{aligned}
& c_{d, s} \int_{B_{t} \times[-t, t]^{k}}\left(\sum_{i=1}^{n} \delta_{\left(x_{i}^{\prime}, 0\right)}^{(\eta)}\left(\left(n^{1 / d} \tilde{x}_{n}, 0\right)+X\right) d X-\mu_{V}^{\prime}\left(n^{1 / d} \tilde{x}_{n}+X\right) \delta_{\mathbb{R}^{d}}\right) \\
& =\int_{\partial\left(B_{t} \times[-t, t]^{k}\right)}|y|^{\gamma} \nabla h_{n, \eta}^{\prime}\left(\left(n^{1 / d} \tilde{x}_{n}, 0\right)+X\right) \cdot \vec{v} .
\end{aligned}
$$

It follows from the above relations and the Cauchy-Schwarz inequality (together with the integrability of $|y|^{\gamma}$ ) that

$$
\left|\int_{B_{t} \times \mathbb{R}^{k}} \sum \delta_{\left(x_{i}^{\prime}, 0\right)}^{(\eta)}\left(\left(n^{1 / d} \tilde{x}_{n}, 0\right)+X\right) d X-\int_{B_{t}} \mu_{V}\left(\tilde{x}_{n}+n^{-1 / d} x\right) d x\right| \leqslant C_{\eta, R},
$$

for some constant that depends on $\eta, R$, and the constant in (5.5). Since $\mu_{V}$ is bounded, it follows that, letting $\left.\underline{v_{n}^{\prime}}:=v_{n}^{\prime}\left(n^{1 / d} \tilde{x}_{n}, 0\right)+\cdot\right)$, we have

$$
\underline{v_{n}^{\prime}}\left(B_{R-1} \times \mathbb{R}^{k}\right) \leqslant C_{d}\left\|\mu_{V}\right\|_{L^{\infty}} R^{d}+C_{\eta, R} .
$$


This establishes that $\left\{v_{n}^{\prime}\right\}$ is locally bounded independently of $n$. In view of the form of $\underline{v_{n}^{\prime}}$, its limit after extraction of a subsequence can only be of the form $v=\sum_{p \in \Lambda} N_{p} \delta_{p}$, where $N_{p}$ are positive integers, and $\Lambda$ is a discrete set contained in $\mathbb{R}^{d} \times\{0\}$.

From the bound (5.5), up to a further extraction, we have that $\underline{\nabla h}_{n, \eta}^{\prime}:=$ $\nabla h_{n, \eta}^{\prime}\left(\left(n^{1 / d} \tilde{x}_{n}, 0\right)+\cdot\right)$ is locally weakly convergent in the weighted space $L_{|y| \gamma}^{2}$, and converges (locally) to some vector field $E_{\eta}$. Using Hölder's inequality, we note that $L_{|y|^{\gamma}}^{2}\left(B_{R}\right)$ (where $B_{R}$ denotes the ball of radius $R$ in $\mathbb{R}^{d+k}$ ) embeds continuously into $L^{q}\left(B_{R}\right)$ for $1<q<\min \left(2, \frac{2}{\gamma+1}\right)$. It thus follows that $\underline{\nabla h}_{n, \eta}^{\prime}$ is bounded in $L_{\text {loc }}^{q}$ and converges to $E_{\eta}$ also in the sense of distributions. We next deduce that $\nabla h_{n}^{\prime}:=$ $\nabla h_{n}^{\prime}\left(\left(n^{1 / 2} \tilde{x}_{n}, 0\right)+\cdot\right)$ is bounded in $L^{p}\left(B_{R}\right)$ for $1<p<\min \left(2, \frac{2}{\gamma+1}, \frac{d+k}{s+1}\right)$. Indeed, $\nabla h_{n}^{\prime}=$ $\nabla h_{n, \eta}^{\prime}-\sum_{i=1}^{n} \nabla f_{\eta}\left(X-\left(x_{i}^{\prime}, 0\right)\right), \nabla f_{\eta}$ is in $L^{p}\left(B_{R}\right)$ for any $p<\frac{d+k}{s+1}$, and the number of $x_{i}^{\prime}$ in $\left(n^{1 / d} \tilde{x}_{n}, 0\right)+B_{R}$ is bounded by $v_{n}^{\prime}\left(B_{R} \times \mathbb{R}^{k}\right)$. We thus deduce that, up to a further extraction, $\underline{\nabla} h_{n}^{\prime}$ converges weakly in $L^{p}\left(B_{R}\right)$, for such $p$, to some vector field $E$, which must be a gradient. Moreover, $E_{\eta}=\Phi_{\eta}(E)$ because $\Phi_{\eta}$ commutes with the weak convergence in $L_{\text {loc }}^{p}$ of $\underline{\nabla h_{n}^{\prime}}$. Indeed, by definition,

$$
\Phi_{\eta}\left(\underline{\nabla h}_{n}^{\prime}\right)=\underline{\nabla h}_{n}^{\prime}+\sum_{p \in \Lambda_{n}} N_{p} \nabla f_{\eta}(.-p),
$$

where $\Lambda_{n}$ is the set of points associated with $v_{n}^{\prime}$. Since all these points have limits, one may check that the sum in the right-hand side converges to $\sum_{p \in \Lambda} N_{p} \nabla f_{\eta}(.-p)$, at least weakly in $L_{\text {loc }}^{p}$. Taking the limit, we deduce that

$$
E_{\eta}=E+\sum_{p \in \Lambda} N_{p} \nabla f_{\eta}(.-p)
$$

i.e. $E_{\eta}=\Phi_{\eta}(E)$ as desired.

It remains to show that (5.8) holds. For that we start from (1.48), and, translating the equation by $n^{1 / d}\left(\tilde{x}_{n}, 0\right)$ and integrating against a smooth compactly supported test function $\varphi$, we find

$$
\int_{\mathbb{R}^{d+k}} \nabla \varphi \cdot \underline{\nabla h_{n, \eta}^{\prime}}|y|^{\gamma}=c_{d, s} \int_{\mathbb{R}^{d+k}} \varphi\left(\sum \delta_{x_{i}^{\prime}-n^{1 / d} \tilde{x}_{n}}^{(\eta)}-\mu_{V}^{\prime}\left(n^{1 / d}\left(\tilde{x}_{n}, 0\right)+\cdot\right) \delta_{\mathbb{R}^{d}}\right) .
$$

In view of the $L_{|y|^{\gamma}}^{2}$ convergence of $\underline{\nabla h}_{n, \eta}^{\prime}$, the weak convergence in measures of $\underline{v}_{n}^{\prime}$, and the continuity of $\mu_{V}$ at $\tilde{x}=\lim _{n \rightarrow \infty} \tilde{x}_{n}$, taking the $n \rightarrow \infty$ limit in this relation yields

$$
\int_{\mathbb{R}^{d+k}} \nabla \varphi \cdot E_{\eta}|y|^{\gamma}=c_{d, s} \int_{\mathbb{R}^{d+k}} \varphi\left(\sum_{p \in \Lambda} N_{p} \delta_{p}^{(\eta)}-\mu_{V}(\tilde{x}) \delta_{\mathbb{R}^{d}}\right),
$$

where $\Lambda, N_{p}$ are associated to $v$. It thus follows that

$$
-\operatorname{div}\left(|y|^{\gamma} E_{\eta}\right)=c_{d, s} \sum_{p \in \Lambda} N_{p} \delta_{p}^{(\eta)}-\mu_{V}(\tilde{x}) \delta_{\mathbb{R}^{d}} \quad \text { in } \mathbb{R}^{d+k}
$$

in the sense of distributions. Since $E=\Phi_{\eta}^{-1}\left(E_{\eta}\right)$, relation (5.8) follows. 
At this point, the rest of the proof is identical to that in [44]. We thus only state the main steps. The assumptions of the abstract Theorem 7 of [48] are satisfied thanks to Lemma 5.1. This theorem then ensures that $P_{n, \eta}$ as defined above is tight and converges up to extraction to a Borel probability measure $P_{\eta}$ on $\mathcal{X}$, and $P:=\Phi_{\eta}^{-1}\left(P_{\eta}\right)$ is admissible (for this we need that $P$-a.e. $(x, E), E \in \mathcal{A}_{\mu_{V}(x)}$. This is ensured by Lemma 5.1, assuming that a.e. point in $\Sigma$ is a point of continuity of $\mu_{V}$. Theorem 7 of [48] also yields that

$$
\liminf _{n \rightarrow 0} \mathbf{F}_{n, \eta}(\mathcal{Y}) \geqslant \int \mathbf{f}_{\eta}(x, \mathcal{Y}) d P_{\eta}(x, \mathcal{Y})=\int\left(\lim _{R \rightarrow+\infty} f_{K_{R}} \mathbf{f}_{\eta}\left(x, \theta_{\lambda} \mathcal{Y}\right) d \lambda\right) d P_{\eta}(x, \mathcal{Y})
$$

The second relation is an application of Wiener's multiparameter ergodic theorem as in $[4,18]$, in view of the translation invariance of $P$ and thus of $P_{\eta}$, and it is part of the result that the limit exists. Finally, combining this with the definition of $\mathbf{f}_{\eta}$ and (5.4), we are led to

$$
\liminf _{n \rightarrow \infty} \frac{1}{|\Sigma| n} \int_{\mathbb{R}^{d+k}}|y|^{\gamma}\left|\nabla h_{n, \eta}^{\prime}\right|^{2} \geqslant \lim _{R \rightarrow \infty} f_{K_{R}}\left|\Phi_{\eta}(E)\right|^{2} d P(x, E) .
$$

Inserting the above inequality into (5.1) and using the fact that $\int \mu_{V}=1$ and the first marginal of $P$ is the normalized Lebesgue measure on $\Sigma$, we obtain

$$
\liminf _{n \rightarrow \infty} n^{-1-\frac{s}{d}}\left(H_{n}\left(x_{1}, \ldots, x_{n}\right)-n^{2} \mathcal{E}\left(\mu_{V}\right)\right) \geqslant \frac{|\Sigma|}{c_{d, s}} \int \mathcal{W}_{\eta}(E) d P(x, E)-C \eta^{\frac{d-s}{2}},
$$

and the analogous statement in the cases (1.3)-(1.4). It then remains to let $\eta \rightarrow 0$. Since $P$-a.e. $(x, E)$ is in $\mathcal{A}_{\mu_{V}(x)}$ and $\mu_{V}$ is bounded, in view of (1.35)-(1.36) and Proposition 2.4, we have that $\mathcal{W}_{\eta}$ is bounded below independently of $\eta$ for $P$-a.e. $(x, E)$. We may thus apply Fatou's lemma to take the $\eta \rightarrow 0$ limit. In view of the definition of $\widetilde{\mathcal{W}},(1.53)$, we thus obtain the following general lower bound result.

Proposition 5.2. Assume that $V$ satisfies (1.8)-(1.10), and that $\mu_{V}$ is a measure with an $L^{\infty}$ density and a.e. continuous. Let $x_{1}, \ldots, x_{n} \in \mathbb{R}^{d}$, and define $P_{v_{n}}$ as in (1.52). Then, up to extraction of a subsequence, $P_{v_{n}}$ converges weakly in the sense of probability measures to a measure $P \in \mathcal{P}(\mathcal{X})$ which is admissible, and

$$
\liminf _{n \rightarrow \infty} n^{-1-\frac{s}{d}}\left(H_{n}\left(x_{1}, \ldots, x_{n}\right)-n^{2} \mathcal{E}\left(\mu_{V}\right)\right) \geqslant \tilde{\mathcal{W}}(P) \text { in case (1.2), }
$$

respectively

$$
\liminf _{n \rightarrow \infty} \frac{1}{n}\left(H_{n}\left(x_{1}, \ldots, x_{n}\right)-n^{2} \mathcal{E}\left(\mu_{V}\right)+\frac{n}{d} \log n\right) \geqslant \tilde{\mathcal{W}}(P) \text { in cases (1.3)-(1.4). }
$$

\section{Screening}

Starting with this section, we turn to the upper bound part of the proof. We now need to use the further assumptions on $\mu_{V},(1.12)-(1.14)$.

In this section, we prove the screening result. More precisely, we consider an 'electric' vector field in a strip $K_{R} \times \mathbb{R}^{k}$ for $R$ large. We would like to prove that, up to errors on the energy which can be made negligible as $R \rightarrow \infty$, we may modify $E$ in a neighborhood 
of the boundary of the strip in such a way as to obtain $E \cdot v=0$ on $\partial K_{R} \times \mathbb{R}^{k}$. This allows us to later patch together several copies of the so-constructed $E$ without creating new divergence. We also want to ensure that the construction preserves the 'good separation' of the points.

Constants $C$ will mean positive constants which may only depend on $d$ and $s$.

Proposition 6.1 (Screening). Let $0<\varepsilon<1 / 2$ be given. Assume that $K_{R} \subset \mathbb{R}^{d} \times\{0\}$ is a hyperrectangle whose sidelengths are in $[2 R, 3 R]$ and such that $\left|K_{R}\right|$ is an integer, and that $E$ is a vector field defined in $\check{K}_{R} \times \mathbb{R}^{k}$ with $\check{K}_{R}:=\left\{x \in K_{R}, \operatorname{dist}\left(x, \partial K_{R}\right) \geqslant \frac{1}{2} \varepsilon R\right\}$ and satisfying

$$
-\operatorname{div}\left(|y|^{\gamma} E\right)=c_{d, s}\left(\sum_{p \in \Lambda} N_{p} \delta_{p}-\delta_{\mathbb{R}^{d}}\right) \quad \text { in } \check{K}_{R} \times \mathbb{R}^{k}
$$

for some discrete set $\Lambda \subset \mathbb{R}^{d}$ and positive integers $N_{p}$. There exists $\eta_{0}>0$ depending only on $d$ such that, for any $0<\eta, \eta^{\prime}<\eta_{0}$, the following holds. Let $E_{\eta}, E_{\eta^{\prime}}$ be associated to $E$ as in (1.29), and let

$$
M_{R, \varepsilon, \eta}:=\frac{1}{R^{d}} \int_{\check{K}_{R} \times\left[-\varepsilon^{2} R, \varepsilon^{2} R\right]^{k}}|y|^{\gamma}\left|E_{\eta}\right|^{2},
$$

and in the case when $k=1$

$$
e_{\varepsilon, R}:=\frac{1}{\varepsilon^{4} R^{d}} \int_{\check{K}_{R} \times\left(\mathbb{R} \backslash\left(-\frac{1}{2} \varepsilon^{2} R, \frac{1}{2} \varepsilon^{2} R\right)\right)}|y|^{\gamma}|E|^{2} .
$$

There exists $R_{0}>0$ such that, if

$$
\begin{aligned}
& R>\max \left(\frac{R_{0}}{\varepsilon^{2}}, \frac{R_{0} M_{R, \varepsilon, \eta^{\prime}}}{\varepsilon^{3}}\right), \\
& R> \begin{cases}R_{0} M_{R, \varepsilon, \eta}^{1 / 2} \varepsilon^{-d-3 / 2} & \text { if } k=0, \\
\max \left(R_{0} M_{R, \varepsilon, \eta}^{1 /(1-\gamma)} \varepsilon^{\frac{-1-2 d+\gamma}{1-\gamma}}, R_{0} \varepsilon^{\frac{2 \gamma}{1-\gamma}} e_{\varepsilon, R}^{1 /(1-\gamma)}\right) & \text { if } k=1,\end{cases}
\end{aligned}
$$

then there exists a vector field $\hat{E} \in L_{\mathrm{loc}}^{p}\left(\mathbb{R}^{d+k}, \mathbb{R}^{d+k}\right.$ ) (with $p$ as in the introduction) such that the following hold.

- $\hat{E} \cdot \vec{v}=0$ on $\partial K_{R} \times \mathbb{R}^{k}$, where $\vec{v}$ is the outer unit normal, and $\hat{E}=0$ outside $K_{R} \times \mathbb{R}^{k}$.

- There exists a subset $\hat{\Lambda} \subset K_{R}$ and positive integers $N_{p}$ such that $\hat{E}$ satisfies

$$
-\operatorname{div}\left(|y|^{\gamma} \hat{E}\right)=c_{s, d}\left(\sum_{p \in \hat{\Lambda}} N_{p} \delta_{p}-\delta_{\mathbb{R}^{d}}\right) \text { in } K_{R} \times \mathbb{R}^{k} .
$$

- $\hat{E}=E \quad$ in $\quad$ a hyperrectangle $\quad K_{R}^{\prime} \times\left[-\varepsilon^{2} R, \varepsilon^{2} R\right]^{k}$, where $K_{R}^{\prime} \quad$ contains $\left\{x \in K_{R}, \operatorname{dist}\left(x, \partial K_{R}\right)>2 \varepsilon R\right\}$. In particular, $\hat{\Lambda} \cap K_{R}^{\prime}=\Lambda \cap K_{R}^{\prime}$.

- The minimal distance between the points in $\hat{\Lambda} \cap\left(K_{R} \backslash K_{R^{\prime}}\right)$ and between them and $\partial K_{R}$ is bounded below by $\eta_{0}$. The minimal distance between points in $\hat{\Lambda}$ counted with 
multiplicity, and between points in $\hat{\Lambda}$ and $\partial K_{R}$ is bounded below by the minimum of $\eta_{0}$ and of the minimal distance between the original points of $\Lambda$ counted with multiplicity. In other words, if the points of $\Lambda$ are simple and well separated, so are those of $\hat{\Lambda}$.

- Letting $\hat{E}_{\eta}$ be $\Phi_{\eta}(\hat{E})$, we have

$$
\int_{K_{R} \times \mathbb{R}^{k}}|y|^{\gamma}\left|\hat{E}_{\eta}\right|^{2} \leqslant\left(\int_{\check{K}_{R} \times \mathbb{R}^{k}}|y|^{\gamma}\left|E_{\eta}\right|^{2}\right)(1+C \varepsilon)+C g(\eta)\left(1+M_{R, \varepsilon, \eta^{\prime}}\right) \varepsilon R^{d}+C e_{\varepsilon, R} \varepsilon R^{d},
$$

where $C$ depends only on s and $d$.

Remark 6.2. In this result, one should think of $M_{R, \varepsilon, \eta}$ as being bounded above by a constant times $g(\eta)$, and $e_{\varepsilon, R}$ as bounded by a constant. Then the conditions of $R$ are that it has to be large enough, so much so as $\varepsilon$ and $\eta$ are small. In a first reading, one may also take $\eta^{\prime}=\eta$ in the statement, and obtain

$$
\int_{K_{R} \times \mathbb{R}^{k}}|y|^{\gamma}\left|\hat{E}_{\eta}\right|^{2} \leqslant\left(\int_{\check{K}_{R} \times \mathbb{R}^{k}}|y|^{\gamma}\left|E_{\eta}\right|^{2}\right)(1+C g(\eta) \varepsilon)+C \varepsilon R^{d} .
$$

Since $\int|y|^{\gamma}\left|E_{\eta}\right|^{2}$ blows up like $g(\eta)$ as $\eta \rightarrow 0$, this gives an additive error in $g(\eta)^{2}$. But one may also prefer to choose say $\eta^{\prime}=1 / 8$ and $\eta$ small and meant to tend to 0 . For a vector field $E \in \mathcal{A}_{1}$ of finite energy, $M_{R, \varepsilon, \eta^{\prime}}$ will be bounded by a constant depending on the choice $1 / 8$ and $\mathcal{W}(E)$. The formulation then gives in that case a bound

$$
\int_{K_{R} \times \mathbb{R}^{k}}|y|^{\gamma}\left|\hat{E}_{\eta}\right|^{2} \leqslant\left(\int_{\check{K}_{R} \times \mathbb{R}^{k}}|y|^{\gamma}\left|E_{\eta}\right|^{2}\right)(1+C \varepsilon)+C g(\eta) \varepsilon R^{d} .
$$

The additive error term then blows up like $g(\eta)$ as $\eta \rightarrow 0$ instead of $g(\eta)^{2}$.

\subsection{Preliminary lemmas}

We start with a series of preliminary results which will be the building blocks for the construction of $\hat{E}$.

Lemma 6.3 (Subdivision of a hyperrectangle). Let $H=\left[0, \ell_{1}\right] \times \cdots \times\left[0, \ell_{d}\right]$ be a $d$-dimensional hyperrectangle of sidelengths $\ell_{i}$. Fix a face $F$ of $H$. Let $m>0$ be such that $m|H| \in \mathbb{N}$, and, for all $i, \ell_{i}>2 m^{-1 / d}$. Then there is a partition of $H$ into $m|H|$ subrectangles $\mathcal{R}_{j}$, such that the following hold.

- All hyper-rectangles have volume $1 / \mathrm{m}$.

- The sidelengths of each $\mathcal{R}_{j}$ lie in the interval $\left[2^{-d} m^{-1 / d}, 2^{d} m^{-1 / d}\right]$.

- All the $\mathcal{R}_{j}$ which have a face in common with $F$ have the same sidelength in the direction perpendicular to $F$.

Note that, in general, even if the area of $H_{i}$ is assumed to be an integer, it is not possible to divide it into congruent hyper-rectangles of sidelengths in $\left[C^{-1}, C\right]$ with a constant $C$ independent of $H_{i}$ (see the lower bound in Theorem 8 of [17] in the case when $d=2$ ), but we may however divide it into hyper-rectangles of comparable sizes. A question which we do not answer is whether one can achieve a bound on their sizes independent of the dimension. 
Proof. The statement is obvious in dimension 1 . We prove the statement for $d \geqslant 2$ by induction on the dimension. Up to relabeling the directions, we may suppose that $F$ is one of the faces where all the first $d-1$ coordinates are constant.

We use the induction hypothesis for the hyperrectangle $H^{\prime}:=\left[0, \ell_{1}\right] \times \cdots \times\left[0, \ell_{d-1}\right]$ with the choice

$$
m^{\prime}=\frac{\left[m^{\frac{d-1}{d}}\left|H^{\prime}\right|\right]}{\left|H^{\prime}\right|}
$$

which does satisfy $m^{\prime}\left|H^{\prime}\right| \in \mathbb{N}$. We note that, from the assumption that $\ell_{i}>2 m^{-1 / d}$, we have $m^{\prime} \in\left[\frac{1}{2} m^{\frac{d-1}{d}}, m^{\frac{d-1}{d}}\right]$. We thus obtain a subdivision into hyper-rectangles $\mathcal{R}_{j}^{\prime}$ of volumes all equal to $1 / m^{\prime}$ and sidelengths belonging to $\left[2^{-(d-1)}\left(m^{\prime}\right)^{-\frac{1}{d-1}}, 2^{d-1}\left(m^{\prime}\right)^{-\frac{1}{d-1}}\right]$. We then split the interval $\left[0, \ell_{d}\right]$ into $\left[\ell_{d} m / m^{\prime}\right]-1$ adjacent equal intervals of length $m^{\prime} / m$, plus a remaining interval of length $\ell^{\prime} \in\left[m^{\prime} / m, 2 m^{\prime} / m\right]$. By taking the Cartesian product of the first group of intervals with the $\mathcal{R}_{j}^{\prime}$, we obtain hyperrectangles of volume $1 / m$, and of sidelengths in $\left[2^{-d} m^{-1 / d}, 2^{d} m^{-1 / d}\right]$.

The remaining strip is of the form $H^{\prime} \times\left[0, \ell^{\prime}\right]$ and has again volume in $\frac{1}{m} \mathbb{N}$. We may apply the induction hypothesis to $H^{\prime}$, this time with $m^{\prime \prime}=m \ell^{\prime}$, since $m^{\prime \prime}\left|H^{\prime}\right|=m \ell^{\prime}\left|H^{\prime}\right| \in$ $\mathbb{N}$. We thus obtain a subdivision of $H^{\prime}$ into hyperrectangles $\mathcal{R}_{j}^{\prime \prime}$ of volume $1 / m^{\prime \prime}$ and sidelengths in $\left[2^{-(d-1)}\left(m^{\prime \prime}\right)^{-\frac{1}{d-1}}, 2^{d-1}\left(m^{\prime \prime}\right)^{-\frac{1}{d-1}}\right]$. Taking the Cartesian products of these hyper-rectangles with $\left[0, \ell^{\prime}\right]$ concludes the proof. The last point of the thesis is also true, because the hyper-rectangles $\mathcal{R}_{j}$ with a face in common with $F$ are all making up the same strip.

Lemma 6.4 (Correcting fluxes on hyper-rectangles). Let $H$ be a hyperrectangle of $\mathbb{R}^{d}$ with sidelengths in $[\ell / 2,3 \ell]$. Let $\tilde{H}:=H \times[-\ell, \ell]^{k}$. Let $g \in L_{|y|^{\gamma}}^{2}(\partial \tilde{H})$, and let $m$ be a function on $H$ of average $m_{0}$ such that

$$
-c_{d, s} m_{0}|H|=\int_{\partial \tilde{H}}|y|^{\gamma} g .
$$

Then the mean-zero solution to

$$
\begin{cases}-\operatorname{div}\left(|y|^{\gamma} \nabla h\right)=c_{d, s} m \delta_{\mathbb{R}^{d}} & \text { in } \tilde{H} \\ \partial_{\nu} h=g & \text { on } \partial \tilde{H}\end{cases}
$$

satisfies

$$
\int_{\tilde{H}}|y|^{\gamma}|\nabla h|^{2} \leqslant C \ell \int_{\partial \tilde{H}}|y|^{\gamma}|g|^{2}+C \ell^{d+1-\gamma}\left\|m-m_{0}\right\|_{L^{\infty}(H)}^{2},
$$

where $C$ depends only on $d$ and $s$.

Proof. First, we note that, in the case when $k=0$ (which goes along with $\gamma=0$ ), this was proven in [44, Lemma 5.8]. We may thus focus on the case when $k=1$.

We may split $h$ as $h_{1}+h_{2}+h_{3}$, where $h_{1}$ solves

$$
\begin{cases}-\operatorname{div}\left(|y|^{\gamma} \nabla h_{1}\right)=c_{d, s} m_{0} \delta_{\mathbb{R}^{d}} & \text { in } \tilde{H}, \\ \partial_{\nu} h_{1}=c:=-\frac{c_{d, s} m_{0}}{2 \ell^{\gamma}} & \text { on } H \times \partial[-\ell, \ell], \\ \partial_{\nu} h_{1}=0 & \text { on the rest of } \partial \tilde{H},\end{cases}
$$


$h_{2}$ solves

$$
\begin{cases}-\operatorname{div}\left(|y|^{\gamma} \nabla h_{2}\right)=0 & \text { in } \tilde{H} \\ \partial_{\nu} h_{2}=g-c & \text { on } H \times \partial[-\ell, \ell] \\ \partial_{\nu} h_{2}=g & \text { on the rest of } \partial \tilde{H}\end{cases}
$$

and $h_{3}$ solves

$$
\begin{cases}-\operatorname{div}\left(|y|^{\gamma} \nabla h_{3}\right)=c_{d, s}\left(m-m_{0}\right) \delta_{\mathbb{R}^{d}} & \text { in } \tilde{H}, \\ \partial_{\nu} h_{3}=0 & \text { on } H \times \partial[-\ell, \ell], \\ \partial_{\nu} h_{3}=0 & \text { on the rest of } \partial \tilde{H},\end{cases}
$$

We note that the first equation has a unique solution up to constants, and an explicit solution is $h_{1}=\lambda m_{0}|y|^{1-\gamma}$, with

$$
\lambda=\frac{c}{m_{0}(1-\gamma)} \ell^{\gamma}=\frac{-c_{s, d}}{2(1-\gamma)} .
$$

Then $\left|\nabla h_{1}\right|=(1-\gamma) \lambda m_{0}|y|^{-\gamma}$, and with straightforward computations

$$
\int_{\tilde{H}}|y|^{\gamma}\left|\nabla h_{1}\right|^{2} \leqslant C m_{0}^{2} \ell^{d+1-\gamma}
$$

with $C$ depending only on $d$ and $s$. Next, we note that $h_{2}$ can be obtained as the minimizer with average 0 of the functional $\frac{1}{2} \int_{\tilde{H}}|y|^{\gamma}|\nabla h|^{2}-\int_{\partial \tilde{H}}|y|^{\gamma} h \bar{g}$, where $\bar{g}$ is the boundary condition. Comparing $h_{2}$ with the choice $h=0$, we obtain, using the Cauchy-Schwarz inequality,

$$
\begin{aligned}
\frac{1}{2} \int_{\tilde{H}}|y|^{\gamma}\left|\nabla h_{2}\right|^{2} & \leqslant \int_{\partial \tilde{H}}|y|^{\gamma}\left|h_{2}\right||\bar{g}| \\
& \leqslant\left(\int_{\partial \tilde{H}}|y|^{\gamma}\left|h_{2}\right|^{2}\right)^{\frac{1}{2}}\left(\int_{\partial \tilde{H}}|y|^{\gamma}|\bar{g}|^{2}\right)^{\frac{1}{2}}
\end{aligned}
$$

and we estimate this in weighted spaces via

$$
\int_{\partial \tilde{H}}|y|^{\gamma}\left|h_{2}\right|^{2} \leqslant C \ell \int_{\tilde{H}}|y|^{\gamma}\left|\nabla h_{2}\right|^{2} .
$$

We postpone the proof of this inequality to the end. Inserting (6.11) into (6.10), we obtain

$$
\int_{\tilde{H}}|y|^{\gamma}\left|\nabla h_{2}\right|^{2} \leqslant C \ell \int_{\partial \tilde{H}}|y|^{\gamma}|\bar{g}|^{2},
$$

and inserting the definition of $\bar{g}$ and $c$, we are led to

$$
\int_{\tilde{H}}|y|^{\gamma}\left|\nabla h_{2}\right|^{2} \leqslant C \ell \int_{\partial \tilde{H}}|y|^{\gamma}|g|^{2}+C m_{0}^{2} \ell^{d+1-\gamma} .
$$

Next, we apply the Cauchy-Schwarz inequality again to obtain from (6.4) that

$$
m_{0}^{2}=\frac{1}{c_{d, s}^{2}|H|^{2}}\left(\int_{\partial \tilde{H}}|y|^{\gamma} g\right)^{2} \leqslant C \ell^{-d+\gamma} \int_{\partial \tilde{H}}|y|^{\gamma}|g|^{2},
$$


and combining this with (6.12), we deduce

$$
\int_{\tilde{H}}|y|^{\gamma}\left|\nabla h_{2}\right|^{2} \leqslant C \ell \int_{\partial \tilde{H}}|y|^{\gamma}|g|^{2} .
$$

For $h_{3}$, we first assume the following trace inequality, whose proof is postponed to the end:

$$
\int_{H}|h(x, 0)|^{2} \leqslant C \ell^{1-\gamma} \int_{\tilde{H}}|y|^{\gamma}|\nabla h|^{2} .
$$

Assuming this, let us multiply (6.8) by $h_{3}$ and integrate by parts to obtain

$$
\int_{\tilde{H}}|y|^{\gamma}\left|\nabla h_{3}\right|^{2}=c_{d, s} \int_{H \times\{0\}}\left(m-m_{0}\right) h_{3} .
$$

Combining this with the Cauchy-Schwarz inequality and (6.14), we easily deduce that

$$
\int_{\tilde{H}}|y|^{\gamma}\left|\nabla h_{3}\right|^{2} \leqslant C \ell^{1-\gamma}\left\|m-m_{0}\right\|_{L^{\infty}(H)}^{2}|H|=C \ell^{d+1-\gamma}\left\|m-m_{0}\right\|_{L^{\infty}(H)}^{2},
$$

which, combined with (6.9) and (6.13), gives the result.

Let us now prove (6.11) and (6.14). First, we may again reduce to the case when $k=1$ (otherwise the inequality is standard). For any function $h$ let us apply for each $y>0$ the standard trace inequality to $h$ on $H \times\{y\}$ to obtain

$$
\int_{\partial H \times\{y\}}\left|h-h_{y}\right|^{2} \leqslant C \ell \int_{H \times\{y\}}\left|\nabla_{x} h\right|^{2},
$$

where $h_{y}$ is the average of $h$ on $H \times\{y\}$. Integrating against $|y|^{\gamma}$ and using the triangle inequality in $L^{2}$, we deduce that

$$
\begin{aligned}
\int_{\partial H \times[-\ell, \ell]}|y|^{\gamma} h^{2} & \leqslant 2 C \ell \int_{\tilde{H}}|y|^{\gamma}|\nabla h|^{2}+2 \int_{\partial H \times[-\ell, \ell]}|y|^{\gamma}\left|h_{y}\right|^{2} \\
& \leqslant C \ell \int_{\tilde{H}}|y|^{\gamma}|\nabla h|^{2}+C \frac{|\partial H|}{|H|} \int_{\tilde{H}}|y|^{\gamma} h^{2},
\end{aligned}
$$

where we used that, by Jensen's inequality, $\left|h_{y}\right|^{2} \leqslant \frac{1}{|H|} \int_{H \times\{y\}} h^{2}$. Next, we note that the Sobolev inequality in weighted spaces for functions of zero average

$$
\int_{\tilde{H}}|y|^{\gamma}|h|^{2} \leqslant C \ell^{2} \int_{\tilde{H}}|y|^{\gamma}|\nabla h|^{2}
$$

holds by [27], since $|y|^{\gamma}$ is an $A_{2}$ weight. This yields

$$
\int_{\partial H \times[-\ell, \ell]}|y|^{\gamma} h^{2} \leqslant C \ell \int_{\tilde{H}}|y|^{\gamma}|\nabla h|^{2} .
$$

Next, we need to prove the same relation in $H \times\{-\ell, \ell\}$. For any $x \in H$, let us denote $h_{x}=f_{[-\ell, \ell]} h(x, \cdot)$. By the Cauchy-Schwarz inequality, we have

$$
\left|h_{x}\right|^{2} \leqslant \frac{1}{4 \ell^{2}} \int_{-\ell}^{\ell}|y|^{\gamma}|h(x, \cdot)|^{2} \int_{-\ell}^{\ell} \frac{d y}{|y|^{\gamma}} \leqslant C \ell^{-1-\gamma} \int_{-\ell}^{\ell}|y|^{\gamma}|h(x, \cdot)|^{2} .
$$


In addition, for each $x \in H$, we may write

$$
\begin{aligned}
\left|h(x, \ell)-h_{x}\right|^{2} & \leqslant\left(\int_{-\ell}^{\ell}\left|\partial_{y} h(x, \cdot)\right|\right)^{2} \\
& \leqslant \int_{-\ell}^{\ell}|y|^{\gamma}\left|\partial_{y} h(x, \cdot)\right|^{2} \int_{-\ell}^{\ell} \frac{d y}{|y|^{\gamma}}=C \ell^{1-\gamma} \int_{-\ell}^{\ell}|y|^{\gamma}|\nabla h(x, \cdot)|^{2} .
\end{aligned}
$$

Integrating this over $x \in H$, we obtain

$$
\int_{H \times\{\ell\}} \ell^{\gamma}\left|h-h_{x}\right|^{2} \leqslant C \ell \int_{\tilde{H}}|y|^{\gamma}|\nabla h|^{2} .
$$

On the other hand, integrating (6.17) over $x \in H$ yields

$$
\int_{H} \ell^{\gamma}\left|h_{x}\right|^{2} \leqslant \ell^{-1} \int_{\tilde{H}}|y|^{\gamma}|h|^{2} \leqslant C \ell \int_{\tilde{H}}|y|^{\gamma}|\nabla h|^{2},
$$

where we used (6.15). With the triangle inequality, we deduce that

$$
\int_{H \times\{\ell\}}|y|^{\gamma}|h|^{2} \leqslant C \ell \int_{\tilde{H}}|y|^{\gamma}|\nabla h|^{2} .
$$

Combining this with (6.16) and using the symmetry, we conclude that (6.11) holds. We then note that we may slightly modify the last proof to obtain (6.14): instead of (6.18), we can write

$$
\left|h(x, 0)-h_{x}\right|^{2} \leqslant\left(\int_{-\ell}^{\ell}\left|\partial_{y} h(x, \cdot)\right|\right)^{2} \leqslant C \ell^{1-\gamma} \int_{-\ell}^{\ell}|y|^{\gamma}|\nabla h(x, \cdot)|^{2} .
$$

On the other hand, as seen above, $\int_{H}\left|h_{x}\right|^{2} \leqslant C \ell^{1-\gamma} \int_{\tilde{H}}|y|^{\gamma}|\nabla h|^{2}$, so integrating over $H$ and using the triangle inequality yields (6.14).

The following lemma is straightforward; therefore we omit the proof.

Lemma 6.5 (Adding a point without flux creation). Let $\mathcal{R}$ be a hyperrectangle in $\mathbb{R}^{d}$ of barycenter 0 and sidelengths in $[a, b]$ with $a, b>0$, let $\tilde{\mathcal{R}}=\mathcal{R} \times[-1,1], m=1 /|\mathcal{R}|$, and let $X \in B\left(0, \frac{1}{2} \min (a, b)\right)$. The mean-zero solution to

$$
\begin{cases}-\operatorname{div}\left(|y|^{\gamma} \nabla h\right)=c_{d, s}\left(\delta_{X}-m \delta_{\mathbb{R}^{d}}\right) & \text { in } \tilde{\mathcal{R}} \\ \partial_{\nu} h=0 & \text { on } \partial \tilde{\mathcal{R}}\end{cases}
$$

satisfies

$$
\left.\lim _{\eta \rightarrow 0}\left|\int_{\tilde{\mathcal{R}}}\right| y\right|^{\gamma}\left|\nabla h_{\eta}\right|^{2}-c_{d, s} g(\eta) \mid \leqslant C
$$

where $C$ depends only on $d, a$, and $b$.

Lemma 6.6 (Completing charges near the boundary). Let $\mathcal{R}$ be a hyperrectangle in $\mathbb{R}^{d}$ of center 0 and sidelengths in $[a, b]$ with $a, b>0$, and let $\tilde{\mathcal{R}}=\mathcal{R} \times[-1,1]^{k}$. Let $F$ be a face of $\mathcal{R}$ and $\tilde{F}:=F \times[-1,1]^{k}$. Let $\Lambda_{\mathcal{R}} \subset \mathbb{R}^{d} \times\{0\}$ be a discrete set of points contained in 
an $\eta$-neighborhood of $\tilde{F}$, with $\eta<1$, and $N_{p} \in \mathbb{N}^{*}$ for $p \in \Lambda_{\mathcal{R}}$. Let $c$ be a constant such that

$$
c \int_{\tilde{F}}|y|^{\gamma}=c_{d, s} \int_{\tilde{\mathcal{R}}} \sum_{p \in \Lambda_{\mathcal{R}}} N_{p} \delta_{p}^{(\eta)} .
$$

The mean-zero solution to

$$
\begin{cases}-\operatorname{div}\left(|y|^{\gamma} \nabla h\right)=c_{d, s} \sum_{p \in \Lambda_{\mathcal{R}}} N_{p} \delta_{p}^{(\eta)} & \text { in } \tilde{\mathcal{R}} \\ \partial_{\nu} h=0 & \text { on } \partial \tilde{\mathcal{R}} \backslash \tilde{F} \\ \partial_{\nu} h=c & \text { on } \tilde{F}\end{cases}
$$

satisfies

$$
\int_{\tilde{\mathcal{R}}}|y|^{\gamma}|\nabla h|^{2} \leqslant C(g(\eta)+1)\left(\sum_{p \in \Lambda_{\mathcal{R}}} N_{p}\right)^{2},
$$

where $C$ depends only on $d, s, a$, and $b$.

Proof. We may write $h=h_{1}+h_{2}$, where

$$
\begin{cases}-\operatorname{div}\left(|y|^{\gamma} \nabla h_{1}\right)=c_{d, s} \sum_{p \in \Lambda_{\mathcal{R}}} N_{p} \delta_{p}^{(\eta)}-c_{s, d} m \delta_{\mathbb{R}^{d}} & \text { in } \tilde{\mathcal{R}} \\ \partial_{\nu} h_{1}=0 & \text { on } \partial \tilde{\mathcal{R}}\end{cases}
$$

and

$$
\begin{cases}-\operatorname{div}\left(|y|^{\gamma} \nabla h_{2}\right)=c_{s, d} m \delta_{\mathbb{R}^{d}} & \text { in } \tilde{\mathcal{R}} \\ \partial_{\nu} h_{2}=0 & \text { on } \partial \tilde{\mathcal{R}} \backslash \tilde{F} \\ \partial_{\nu} h_{2}=c & \text { on } \tilde{F}\end{cases}
$$

where $m$ is chosen so that

$$
c_{s, d} m|\mathcal{R}|=c_{d, s} \int_{\tilde{\mathcal{R}}} \sum_{p \in \Lambda_{\mathcal{R}}} N_{p} \delta_{p}^{(\eta)}=c \int_{\tilde{F}}|y|^{\gamma} .
$$

In view of Lemma 6.4 (or its proof) we have that

$$
\int_{\tilde{\mathcal{R}}}|y|^{\gamma}\left|\nabla h_{2}\right|^{2} \leqslant C c^{2} \leqslant C\left(\sum_{p \in \Lambda_{\mathcal{R}}} N_{p}\right)^{2}
$$

where $C$ may depend on $d, a$, and $b$, and we used (6.19) to bound $c$. It remains to control $h_{1}$. First, we note that $h_{1}(X)=\sum_{p \in \Lambda_{\mathcal{R}}} N_{p} G_{\eta}(X, p)$, where, for $p \in \mathbb{R}^{d} \times\{0\}, G_{\eta}(X, p)$ denotes the solution with zero average on $\mathcal{R} \times\{0\}$ of

$$
\begin{cases}-\operatorname{div}\left(|y|^{\gamma} \nabla G_{\eta}\right)=c_{s, d}\left(\delta_{p}^{(\eta)}-\left(f_{\tilde{\mathcal{R}}} \delta_{p}^{(\eta)}\right) \delta_{\mathbb{R}^{d}}\right) & \text { in } \tilde{\mathcal{R}} \\ \partial_{\nu} G_{\eta}=0 & \text { on } \partial \tilde{\mathcal{R}} .\end{cases}
$$

The desired estimate will thus follow provided we show that

$$
\int_{\tilde{\mathcal{R}}}|y|^{\gamma}\left|\nabla G_{\eta}\right|^{2} \leqslant C(g(\eta)+1) \text {. }
$$


We recall that the truncated function $g_{\eta}(X)=\min (g(X), g(\eta))=g(X)-f_{\eta}(X)$ is a solution to $-\operatorname{div}\left(|y|^{\gamma} \nabla g_{\eta}\right)=\delta_{0}^{(\eta)}$. We may thus compare $G_{\eta}$ with $g_{\eta}(x-p)$. If $p$ is at distance $>\frac{a}{4}$ from the boundary of $\mathcal{R}$, then $g_{\eta}(x-p)$ has Neumann derivative $\varphi$ which is bounded on $\partial \tilde{\mathcal{R}}$, and $G_{\eta}-g_{\eta}(x-p)$ is a solution to the equation

$$
\begin{cases}-\operatorname{div}\left(|y|^{\gamma} \nabla u\right)=-c_{s, d}\left(f_{\tilde{\mathcal{R}}} \delta_{p}^{(\eta)}\right) \delta_{\mathbb{R}^{d}} & \text { in } \tilde{\mathcal{R}} \\ \partial_{\nu} u=\varphi & \text { on } \partial \tilde{\mathcal{R}}\end{cases}
$$

with $\varphi$ bounded. In view of Lemma 6.4 we thus have

$$
\int_{\tilde{\mathcal{R}}}|y|^{\gamma}\left|\nabla\left(G_{\eta}-g_{\eta}\right)\right|^{2} \leqslant C
$$

where $C$ depends only on $d, a$, and $b$. In that case, the result follows easily, since one may compute that $\int_{\tilde{\mathcal{R}}}|y|^{\gamma}\left|\nabla g_{\eta}\right|^{2}=c_{d, s} g(\eta)+C$.

We now turn to the case where $p$ is close to the boundary of $\mathcal{R}$.

Without loss of generality we may assume that one vertex of $\mathcal{R}$ is at the origin in $\mathbb{R}^{d}$ and that it is the one that $p$ is closest to. We then consider the $2^{d}-1$ points $p_{1}, \ldots, p_{2^{d}}$ obtained by symmetry of $p_{1}:=p$ with respect to the coordinate axes in $\mathbb{R}^{d}$. In other words, if $\left(p^{1}, \ldots, p^{d}\right)$ are the coordinates of $p$, we let the $p_{i}$ be all the points with each $j$ th coordinate equal $\pm p^{j}$. We define $\mathcal{R}^{\prime}$ to be the hyper-rectangle obtained by taking all the same reflections of $\mathcal{R}$ (its sidelengths are thus double those of $\mathcal{R}$, and $\tilde{\mathcal{R}}^{\prime}=\mathcal{R}^{\prime} \times[-1,1]^{k}$. We may write $\mathcal{R}^{\prime}=\bigcup_{i=1}^{2^{d}} \mathcal{R}_{i}$, where $\mathcal{R}_{i}$ is the image of $\mathcal{R}$ by the same symmetry that mapped $p$ to $p^{i}$. We also let $\tilde{\mathcal{R}}_{i}=\mathcal{R}_{i} \times[-1,1]^{k}$.

We can extend $G_{\eta}$ by multireflection to a function on $\tilde{\mathcal{R}}^{\prime}$, and write it as $u_{\eta}+v_{\eta}$, where

$$
\begin{cases}-\operatorname{div}\left(|y|^{\gamma} \nabla u_{\eta}\right)=c_{s, d}\left(\sum_{i=1}^{2^{d}} \delta_{p^{i}}^{(\eta)} \mathbb{1}_{\tilde{\mathcal{R}}_{i}}-2^{d} \mathbb{1}_{\tilde{K}_{a}} \frac{1}{\left|\tilde{K}_{a}\right|}\left(\int_{\tilde{\mathcal{R}}} \delta_{p}^{(\eta)}\right) \delta_{\mathbb{R}^{d}}\right) & \text { in } \tilde{\mathcal{R}}^{\prime}, \\ \partial_{\nu} u_{\eta}=0 & \text { on } \partial \tilde{\mathcal{R}}^{\prime},\end{cases}
$$

where $\tilde{K}_{a}$ is a cube centered at the origin of sidelengths $\min \left(a / 2, \frac{1}{2}\right)$ (recall that we assume that the point $p$ is included in $\tilde{K}_{a}$ ),

$$
\begin{cases}-\operatorname{div}\left(|y|^{\gamma} \nabla v_{\eta}\right)=c_{s, d}\left(\left(2^{d} \mathbb{1}_{\tilde{K}_{a}} \frac{1}{\left|\tilde{K}_{a}\right|}-2^{d} \mathbb{1}_{\tilde{\mathcal{R}}^{\prime}} \frac{1}{\left|\tilde{\mathcal{R}}^{\prime}\right|}\right)\left(\int_{\tilde{\mathcal{R}}^{\prime}} \delta_{p}^{(\eta)}\right)\right) \delta_{\mathbb{R}^{d}} & \text { in } \tilde{\mathcal{R}}^{\prime}, \\ \partial_{\nu} v_{\eta}=0 & \text { on } \partial \tilde{\mathcal{R}}^{\prime} .\end{cases}
$$

By the estimate in Lemma 6.4, we have $\int_{\tilde{\mathcal{R}}^{\prime}}|y|^{\gamma}\left|\nabla v_{\eta}\right|^{2} \leqslant C$, with $C$ depending only on $d$ and $s$. Thus it remains to estimate the same for $u_{\eta}$. For this we build subsolutions and supersolutions to equation (6.24).

For a supersolution, we take $\bar{G}_{\eta}:=\sum_{i=1}^{2^{d}} g_{\eta}\left(x-p_{i}\right)$, which satisfies

$$
-\operatorname{div}\left(|y|^{\gamma} \nabla \bar{G}_{\eta}\right)=c_{d, s} \sum_{i=1}^{2^{d}} \delta_{p_{i}}^{(\eta)} \geqslant-\operatorname{div}\left(|y|^{\gamma} \nabla u_{\eta}\right) \text { in } \tilde{\mathcal{R}}^{\prime}
$$


For a subsolution, we use the explicit solution $h_{M}=\frac{c_{d, s}}{2(1-\gamma)} M|y|^{1-\gamma}$ as in the proof of Lemma 6.4, but with $M=-c_{d, s} 2^{d} /\left|\tilde{K}_{a}\right| \geqslant \frac{c_{d, s}}{|\mathcal{R}|}$, which is bounded since $\gamma<1$ and satisfies

$$
-\operatorname{div}\left(|y|^{\gamma} \nabla\left(u_{\eta}-h_{M}\right)\right) \leqslant 0 .
$$

By the maximum principle lemma, Lemma 4.2 , we deduce that $\bar{G}_{\eta}-u_{\eta}$ and $u_{\eta}-h_{M}$ achieve their minimum on the boundary of $\tilde{\mathcal{R}}^{\prime}$. Up to adding a constant to $u_{\eta}$, we also assume that its minimum in $\tilde{\mathcal{R}}^{\prime}$ is 0 . We may thus write

$$
h_{M}+\min _{\partial \tilde{\mathcal{R}}^{\prime}}\left(u_{\eta}-h_{M}\right) \leqslant u_{\eta} \leqslant \bar{G}_{\eta}-\min _{\partial \tilde{\mathcal{R}}^{\prime}}\left(\bar{G}_{\eta}-u_{\eta}\right),
$$

which yields, in view of the properties of $\bar{G}_{\eta}$ and $h_{M}$,

$$
0 \leqslant u_{\eta} \leqslant C+2^{d} g(\eta)+\max _{\partial \tilde{\mathcal{R}}^{\prime}} u_{\eta}-\min _{\partial \tilde{\mathcal{R}}^{\prime}} u_{\eta}
$$

But $u_{\eta}$ being the solution of (6.24) whose right-hand side is 0 in $\tilde{\mathcal{R}}^{\prime} \backslash \tilde{K}_{a}$ satisfies a Harnack principle (see [27, Theorem 2.3.8]), which asserts that $\max _{K} u_{\eta} \leqslant A \min _{K} u_{\eta}$ for each compact set $K$, and for some constant $A>0$ independent of $u_{\eta}$. Since we have zero Neumann boundary condition, we can in principle extend the solution by reflection across the boundary, so this relation holds in fact up to the boundary of $\tilde{\mathcal{R}}^{\prime}$, and hence for some $K$ containing $\partial \tilde{\mathcal{R}}^{\prime}$, and we now consider such a $K$. Standard arguments then imply that

$$
\max _{\partial \tilde{\mathcal{R}}^{\prime}} u_{\eta}-\min _{\partial \tilde{\mathcal{R}}^{\prime}} u_{\eta} \leqslant(1-\theta)\left(\max _{\tilde{\mathcal{R}}^{\prime}} u_{\eta}-\min _{\tilde{\mathcal{R}}^{\prime}} u_{\eta}\right)=(1-\theta) \max _{\tilde{\mathcal{R}}^{\prime}} u_{\eta}
$$

for $\theta=\frac{1}{1+A}<1$, where $A$ is the constant in the Harnack inequality. Indeed, either $u_{\eta} \geqslant \theta \max _{\tilde{\mathcal{R}}} u_{\eta}$ in $K$, in which case we deduce that $\max _{K} u_{\eta}-\min _{K} u_{\eta} \leqslant(1-\theta) \max _{\tilde{\mathcal{R}}} u_{\eta}$ and this implies (6.27), or else $\min _{K} u_{\eta} \leqslant \theta \max _{\tilde{\mathcal{R}}} u_{\eta}$, in which case the above Harnack inequality yields $\max _{K} u_{\eta} \leqslant A \theta \max _{\tilde{\mathcal{R}}} u_{\eta}=(1-\theta) \max _{\tilde{\mathcal{R}}} u_{\eta}$ by choice of $\theta$, which again implies (6.27).

Inserting then (6.27) into (6.26), we deduce that $\theta \max _{\tilde{\mathcal{R}}^{\prime}} u_{\eta} \leqslant C+2^{d} g(\eta)$, i.e.

$$
0 \leqslant u_{\eta} \leqslant C(1+g(\eta)) \text { in } \tilde{\mathcal{R}}^{\prime}
$$

for some positive constant $C$ depending only on $d$ and $s$. Multiplying (6.24) by $u_{\eta}$, integrating by parts, and inserting this bound, we find

$$
\int_{\tilde{\mathcal{R}}^{\prime}}|y|^{\gamma}\left|\nabla u_{\eta}\right|^{2}=c_{d, s} \int_{\tilde{\mathcal{R}}^{\prime}} u_{\eta}\left(\sum_{i=1}^{2^{d}} \delta_{p^{i}}^{(\eta)}-2^{d} \mathbb{1}_{\tilde{K}_{a}} \frac{1}{\left|\tilde{K}_{a}\right|}\left(\int_{\tilde{\mathcal{R}}} \delta_{p}^{(\eta)}\right) \delta_{\mathbb{R}^{d}}\right) \leqslant C(1+g(\eta)),
$$

where again $C$ depends only on $d$ and $s$. Combining this with the estimate on $v_{\eta}$, we deduce that $\int_{\tilde{\mathcal{R}}}|y|^{\gamma}\left|\nabla G_{\eta}\right|^{2} \leqslant C(1+g(\eta))$ with $C$ depending only on $s$ and $d$, which is the desired estimate (6.23). 


\subsection{Proof of Proposition 6.1}

Step 1. Finding a good boundary.

We note that there exist a constant $C>0$ depending only on $d$ and a hyperrectangle $K_{R}^{\prime} \subset \check{K}_{R} \subset K_{R}$ whose faces are at distance $t \in\left[\frac{1}{2} \varepsilon R, \varepsilon R\right]$ from those of $\check{K}_{R}$ and parallel to them, such that, denoting by $\left(\partial K_{R}^{\prime}\right)_{L}$ the $L$-tubular neighborhood of its boundary and assuming that $\frac{1}{2} \varepsilon R>2^{d+2}$ (which may be included in the condition (6.2)), we have

$$
\begin{gathered}
\int_{\partial K_{R}^{\prime} \times\left[-\varepsilon^{2} R, \varepsilon^{2} R\right]^{k}}|y|^{\gamma}\left|E_{\eta}\right|^{2} \leqslant C M_{R, \varepsilon, \eta} \varepsilon^{-1} R^{d-1}, \\
\int_{\left(\partial K_{R}^{\prime}\right)_{2} d+2 \times\left[-\varepsilon^{2} R, \varepsilon^{2} R\right]^{k}}|y|^{\gamma}\left|E_{\eta^{\prime}}\right|^{2} \leqslant C M_{R, \varepsilon, \eta^{\prime}} \varepsilon^{-1} R^{d-1} .
\end{gathered}
$$

Indeed, we obtain that

$$
\int_{\left(\partial K_{R}^{\prime}\right)_{2 d+2} \times\left[-\varepsilon^{2} R, \varepsilon^{2} R\right]^{k}}|y|^{\gamma}\left|E_{\eta^{\prime}}\right|^{2} \leqslant C M_{R, \varepsilon, \eta^{\prime}} \varepsilon^{-1} R^{d-1}
$$

hold simultaneously for $\eta$ and $\eta^{\prime}$ by a pigeonhole principle on a subdivision of $\left[\frac{1}{2} \varepsilon R, \varepsilon R\right]$ into $2^{-(d+4)} \varepsilon R$ pieces of size $2^{d+2}$. We then use the mean-value principle to get (6.28) (translating the faces of $\partial K_{R}^{\prime}$ if necessary). We note that $K_{R}^{\prime}$ contains $\left\{x \in K_{R}, \operatorname{dist}\left(x, \partial K_{R}\right) \geqslant 2 \varepsilon R\right\}$ as desired.

In the case when $k=1$, by a mean-value argument on (6.1), we find that there exists $\ell \in\left[\frac{1}{2} \varepsilon^{2} R, \varepsilon^{2} R\right]$ such that

$$
\int_{K_{R}^{\prime} \times\{-\ell, \ell\}}|y|^{\gamma}|E|^{2}<2 e_{\varepsilon, R} \varepsilon^{2} R^{d-1} .
$$

In what follows, we choose this $\ell$, or in the case when $k=0$ we choose $\ell=\varepsilon^{2} R$. In all cases we have $\ell \leqslant \varepsilon^{2} R$. We also note that, by assumption (6.2), we have $R>\varepsilon R>\ell>1$.

Step 2. Subdividing the domain.

We consider the following regions, on each of which we will perform different constructions to build $\hat{E}$ :

$$
\begin{aligned}
& D_{0}=K_{R}^{\prime} \times[-\ell, \ell]^{k}, \\
& D_{\partial}=\left(K_{R} \times[-\ell, \ell]^{k}\right) \backslash D_{0}, \\
& D_{1}=\left(K_{R} \times[-R, R]\right) \backslash\left(D_{0} \cup D_{\partial}\right) .
\end{aligned}
$$

Of course the set $D_{1}$ does not exist in the case when $k=0$. We let $\Lambda_{0} \subset \Lambda$ be given by those points whose smeared charges touch $\partial K_{R}^{\prime}$, i.e.

$$
\Lambda_{0}=\left\{p \in \Lambda: B(p, \eta) \cap\left(\partial K_{R}^{\prime}\right) \neq \emptyset\right\} .
$$

We may also denote $\Lambda_{\text {int }}=\left(\Lambda \cap K_{R}^{\prime}\right) \backslash \Lambda_{0}$. The goal of the construction is to 'complete' outside $D_{0}$ the smeared-out charges whose centers belong to $\Lambda_{0}$, and place an additional

$$
N_{\partial}:=\left|K_{R}\right|-\left|K_{R}^{\prime}\right|+\frac{1}{c_{d, s}} \int_{\partial D_{0}}|y|^{\gamma} E_{\eta} \cdot \vec{v}-\int_{D_{0}^{c}} \sum_{p \in \Lambda_{0}} N_{p} \delta_{p}^{(\eta)}
$$


points in the set $K_{R} \backslash K_{R}^{\prime}$. Integrating the relation satisfied by $E_{\eta}$ over $D_{0}$ yields that

$$
\begin{aligned}
-\int_{\partial D_{0}}|y|^{\gamma} E_{\eta} \cdot \vec{v} & =c_{d, s} \int_{D_{0}} \sum_{p \in \Lambda} N_{p} \delta_{p}^{(\eta)}-c_{d, s}\left|K_{R}^{\prime}\right| \\
& =c_{d, s} \sum_{p \in \Lambda_{i n t} \cup \Lambda_{0}} N_{p}-c_{d, s} \int_{D_{0}^{c}} \sum_{p \in \Lambda_{0}} N_{p} \delta_{p}^{(\eta)}-c_{d, s}\left|K_{R}^{\prime}\right|,
\end{aligned}
$$

with $\vec{v}$ the outer normal, and hence

$$
N_{\partial}=\left|K_{R}\right|-\sum_{p \in \Lambda_{\text {int }} \cup \Lambda_{0}} N_{p},
$$

which proves, since $\left|K_{R}\right| \in \mathbb{N}$, that $N_{\partial}$ is indeed an integer.

In the case when $k=1$ we also define the constant $C_{0}$ to be

$$
\begin{aligned}
C_{0} & =\frac{\ell^{-\gamma}}{2\left(\left|K_{R}\right|-\left|K_{R}^{\prime}\right|\right)} \int_{D_{0} \times \partial[-\ell, \ell]}|y|^{\gamma} E_{\eta} \cdot \vec{v} \\
& =\frac{\ell^{-\gamma}}{2\left(\left|K_{R}\right|-\left|K_{R}^{\prime}\right|\right)}\left(c_{d, s} N_{\partial}-\int_{\partial K_{R}^{\prime} \times[-\ell, \ell]}|y|^{\gamma} E_{\eta} \cdot \vec{v}+c_{d, s} \int_{D_{0}^{c}} \sum_{p \in \Lambda_{0}} N_{p} \delta_{p}^{(\eta)}\right)-\frac{1}{2} \ell^{-\gamma},
\end{aligned}
$$

where for the second equality we have used (6.32). Whenever $C_{0}$ appears below, we will mean $C_{0}=0$ in the case when $k=0$.

Next, we split $K_{R} \backslash K_{R}^{\prime}$ into hyperrectangles $H_{i}$ with sidelengths $\in[\ell / 2,2 \ell]$ (this can be done by constructing successive strips, in the style of the proof of Lemma 6.3). We then let $\tilde{H}_{i}=H_{i} \times[-\ell, \ell]^{k}$. We also let

$$
n_{i}=c_{d, s} \int_{\tilde{H}_{i}} \sum_{p \in \Lambda_{0}} N_{p} \delta_{p}^{(\eta)}
$$

and let $m_{i}$ be such that

$$
c_{d, s}\left(m_{i}-1\right)\left|H_{i}\right|=\int_{\partial D_{0} \cap \partial \tilde{H}_{i}}|y|^{\gamma} E_{\eta} \cdot \vec{v}-2 C_{0} \ell^{\gamma}\left|H_{i}\right|-n_{i} .
$$

We note that $n_{i}=0$ unless $H_{i}$ has a face in common with $\partial D_{0}$. We will check below that $\left|m_{i}-1\right|<\frac{1}{2}$. Since the sidelengths of $H_{i}$ are of order $\ell$, as soon as $\ell$ is large enough, modifying the boundaries of the $H_{i}$ a little bit, we can ensure that in addition each $m_{i}\left|H_{i}\right| \in \mathbb{N}$. Indeed, combining (6.34) and (6.32), we may check that $\sum_{i} m_{i}\left|H_{i}\right|=N_{\partial}$, and hence is an integer. Once we prove later that $\left|m_{i}-1\right|<\frac{1}{2}$, this will imply that $N_{\partial}>0$.

Since $m_{i} \in\left[\frac{1}{2}, \frac{3}{2}\right]$ and $m_{i}\left|H_{i}\right| \in \mathbb{N}$, if $\ell>\ell_{0}=2\left(\frac{1}{2}\right)^{-1 / d}$, we may apply Lemma 6.3. This places a condition on $\varepsilon^{2} R$ being large enough, which is fulfilled by taking $R_{0}$ large enough in (6.2). For the $\tilde{H}_{i}$ which have some codimension-1 face $F_{i}$ in common with $\partial D_{0}$, we choose such an $F_{i}$ to play the role of $F$ in the notation of Lemma 6.3. Each $H_{i}$ is then divided into hyper-rectangles $\mathcal{R}_{\alpha}, \alpha \in I_{i}$ of sidelengths bounded above and below by 
positive constants which depend only on $d$, and volumes $1 / m_{i}$. We let $\tilde{\mathcal{R}}_{\alpha}=\mathcal{R}_{\alpha} \times[-1,1]^{k}$. For each $\mathcal{R}_{\alpha}$, we denote

$$
\bar{n}_{\alpha}=c_{d, s} \int_{\tilde{\mathcal{R}}_{\alpha}} \sum_{p \in \Lambda_{0}} N_{p} \delta_{p}^{(\eta)}
$$

so that $n_{i}=\sum_{\alpha \in I_{i}} \bar{n}_{\alpha}$.

Step 3. Defining $\hat{E}_{\eta}$.

Over each $\tilde{H}_{i}$ we define $\hat{E}_{\eta}$ as a sum $E_{i, 1}+E_{i, 2}+E_{i, 3}+E_{i, 4}$, some of these terms being zero, except for $H_{i}$ that has some boundary in common with $\partial D_{0}$.

The first vector field contains the contribution of the completion of the smeared charges belonging to $\Lambda_{0}$. We let

$$
E_{i, 1}:=\sum_{\alpha \in I_{i, \partial}} \mathbb{1}_{\tilde{\mathcal{R}}_{\alpha}} \nabla h_{1, \alpha},
$$

where $h_{1, \alpha}$ is the solution of

$$
\begin{cases}-\operatorname{div}\left(|y|^{\gamma} \nabla h_{1, \alpha}\right)=c_{d, s} \sum_{p \in \Lambda_{\alpha}} N_{p} \delta_{p}^{(\eta)} & \text { in } \tilde{\mathcal{R}}_{\alpha}, \\ \partial_{\nu} h_{1, \alpha}=0 & \text { on } \partial \tilde{\mathcal{R}}_{\alpha} \backslash \partial D_{0}, \\ \partial_{\nu} h_{1, \alpha}=\frac{-\bar{n}_{\alpha}}{\int_{\partial \tilde{\mathcal{R}}_{\alpha} \cap \partial D_{0}}|y|^{\gamma}} & \text { on } \partial \tilde{\mathcal{R}}_{\alpha} \cap \partial D_{0} .\end{cases}
$$

We note that the definition of $\bar{n}_{\alpha}$ makes this equation solvable, and that by construction of the $\mathcal{R}_{\alpha}$, the constant $\int_{\partial \tilde{\mathcal{R}}_{\alpha} \cap \partial D_{0}}|y|^{\gamma}$ is bounded above and below by positive constants depending only on $d$ and $s$.

The second vector field is defined to be $E_{i, 2}=\nabla h_{2}$ with

$$
\begin{cases}-\operatorname{div}\left(|y|^{\gamma} \nabla h_{2}\right)=-2 \ell^{\gamma} C_{0} \delta_{\mathbb{R}^{d}} & \text { in } \tilde{H}_{i}, \\ \partial_{\nu} h_{2}=C_{0} & \text { on } H_{i} \times \partial[-\ell, \ell], \\ \partial_{\nu} h_{2}=0 & \text { on the rest of } \partial \tilde{H}_{i} .\end{cases}
$$

Of course, $E_{i, 2}=0$ in the case when $k=0$.

The third vector field is defined to be $E_{i, 3}=\nabla h_{3}$, with

$$
\begin{cases}-\operatorname{div}\left(|y|^{\gamma} \nabla h_{3}\right)=\left(c_{d, s}\left(m_{i}-1\right)+2 \ell^{\gamma} C_{0}\right) \delta_{\mathbb{R}^{d}} & \text { in } \tilde{H}_{i}, \\ \partial_{\nu} h_{3}=0 & \text { on } H_{i} \times \partial[-\ell, \ell]^{k}, \\ \partial_{\nu} h_{3}=g_{i} & \text { on } \partial H_{i} \times[-\ell, \ell]^{k},\end{cases}
$$

where we let $g_{i}=0$ if $\tilde{H}_{i}$ has no face in common with $\partial D_{0}$, and otherwise

$$
g_{i}=-E_{\eta} \cdot \vec{v}+\sum_{\alpha \in I_{i}} \mathbb{1}_{\tilde{\mathcal{R}}_{\alpha}} \frac{\bar{n}_{\alpha}}{\int_{\partial \tilde{\mathcal{R}}_{\alpha} \cap \partial D_{0}}|y|^{\gamma}},
$$

with $E_{\eta} \cdot \vec{v}$ taken with respect to the outer normal to $\partial D_{0}$. We note that this is solvable in view of (6.34) and the definition of $\bar{n}_{\alpha}$ and $n_{i}$. 
Finally, the fourth vector field consists of contributions from almost equally spaced and screened charges over the $\mathcal{R}_{\alpha}$. To define it, let $p_{\alpha}$ be the barycenter of each hyperrectangle $\tilde{\mathcal{R}}_{\alpha}, \alpha \in I_{i}$, and define a function $h_{4, \alpha}$ as solving

$$
\begin{cases}-\operatorname{div}\left(|y|^{\gamma} \nabla h_{4, \alpha}\right)=c_{s, d}\left(\delta_{p_{\alpha}}^{(\eta)}-m_{i} \delta_{\mathbb{R}^{d}}\right) & \text { in } \tilde{\mathcal{R}}_{\alpha} \\ \partial_{\nu} h_{4, \alpha}=0 & \text { on } \partial \tilde{\mathcal{R}}_{\alpha}\end{cases}
$$

We note that this equation is solvable because we have chosen $\left|\mathcal{R}_{\alpha}\right|=1 / m_{i}$. We then define in $\tilde{H}_{i}$

$$
E_{i, 4}=\sum_{\alpha \in I_{i}} \mathbb{1}_{\tilde{\mathcal{R}}_{\alpha}} \nabla h_{4, \alpha}
$$

This finishes defining $\hat{E}_{\eta}$ over $D_{\partial}$. To define $\hat{E}_{\eta}$ over $D_{1}$ (in the case when $k=1$ ) we let $\hat{E}_{\eta}=\nabla h$, with

$$
\begin{cases}-\operatorname{div}\left(|y|^{\gamma} \nabla h\right)=0 & \text { in } D_{1}, \\ \partial_{\nu} h=-\phi & \text { on } \partial D_{1}\end{cases}
$$

where

$$
\phi:=\mathbb{1}_{\partial D_{1} \cap \partial D_{0}} E_{\eta} \cdot \vec{v}+\mathbb{1}_{\partial D_{\partial} \cap \partial D_{1}} C_{0},
$$

with the outer normal taken to be outer to $D_{0}$. Again this equation is solvable in view of (6.33). This completes the construction of $\hat{E}_{\eta}$.

We note that the normal components are always constructed to be continuous across interfaces, so that no divergence is created there, and so $\hat{E}_{\eta}$ thus defined (and extended by 0 outside of $\left.D_{0} \cup D_{\partial} \cup D_{1}\right)$ satisfies

$$
\begin{cases}-\operatorname{div}\left(|y|^{\gamma} \hat{E}_{\eta}\right)=c_{s, d}\left(\sum_{p \in \hat{\Lambda}} N_{p} \delta_{p}^{(\eta)}-\delta_{\mathbb{R}^{d}}\right) & \text { in } K_{R} \times \mathbb{R}^{k}, \\ \hat{E}_{\eta} \cdot v=0 & \text { on } \partial K_{R} \times \mathbb{R}^{k},\end{cases}
$$

where

$$
\hat{\Lambda}=\left(\Lambda \cap K_{R}^{\prime} \backslash \Lambda_{0}\right) \cup \Lambda_{0} \cup\left(\bigcup_{i} \bigcup_{\alpha \in I_{i}}\left\{p_{\alpha}\right\}\right) .
$$

We note that by construction the distance between the new points of $\hat{\Lambda}$ and between them and $\partial K_{R}$ and them and the original points is bounded below by a constant depending only on the sidelengths of $\mathcal{R}_{i}$, and hence on $d$; call it $2 \eta_{0}$. So if the original points of $\Lambda$ are simple and well separated, so are those of $\hat{\Lambda}$. We then define

$$
\hat{E}=\Phi_{\eta}^{-1}\left(\hat{E}_{\eta}\right)=\hat{E}_{\eta}+\sum_{p \in \Lambda_{R, \eta}} N_{p} \nabla f_{\eta}(x-p),
$$

where $f_{\eta}$ is as in (1.25). Since $\hat{\Lambda}$ is at distance $\geqslant \eta_{0}$ from $\partial K_{R}$, and since $f_{\eta}$ is supported in $B(0, \eta)$ with $\eta<\eta_{0}$, we have that $\hat{E}=\hat{E}_{\eta}$ at distance $\geqslant \eta$ from $\hat{\Lambda}$, and in particular 
on $\partial\left(D_{0} \cup D_{\partial}\right)$. In particular, $\hat{E}$ solves

$$
\begin{cases}-\operatorname{div}\left(|y|^{\gamma} \hat{E}\right)=c_{s, d}\left(\sum_{p \in \hat{\Lambda}} N_{p} \delta_{p}-\delta_{\mathbb{R}^{d}}\right) & \text { in } K_{R} \times \mathbb{R}^{k}, \\ \hat{E} \cdot \vec{v}=0 & \text { on } \partial K_{R} \times \mathbb{R}^{k},\end{cases}
$$

as desired. We note that we may as well choose for $p_{\alpha}$, instead of the barycenter of the $\widetilde{\mathcal{R}}_{\alpha}$, any point at distance $<\eta_{0} / 2$ from it, and have the same conclusions verified if $\eta<\eta_{0}$.

Step 4. Controlling the constants.

To control $C_{0}$, we use (6.30) to obtain with the Cauchy-Schwarz inequality, from (6.33),

$$
\left|C_{0}\right| \leqslant C \ell^{-\gamma} R^{-d} \varepsilon^{-1} e_{\varepsilon, R}^{1 / 2} \varepsilon R^{\frac{d-1}{2}} \ell^{\gamma / 2} R^{d / 2}=C e_{\varepsilon, R}^{1 / 2} \varepsilon^{-\gamma} R^{-\frac{\gamma+1}{2}} .
$$

To control $\bar{n}_{\alpha}$, we note that $\left|\bar{n}_{\alpha}\right| \leqslant C n_{\alpha}$, where $n_{\alpha}:=\sum_{p \in \Lambda_{0}, \operatorname{dist}\left(p, \mathcal{R}_{\alpha}\right) \leqslant \eta} N_{p}$. Since the sidelengths of $\mathcal{R}_{\alpha}$ are bounded by $2^{d}\left|m_{i}\right|^{-1 / d}$ and $m_{i}>\frac{1}{2}$, we may choose $L \geqslant 1$ such that the $L$-fattened hyper-rectangles $\left(\mathcal{R}_{\alpha}\right)_{L}$ are contained in $\left(\partial K_{R}^{\prime}\right)_{2^{d+2}}$. Combining Lemma 2.2 with (6.29), and recalling that $p_{\alpha}$ is the barycenter of $\mathcal{R}_{\alpha}$, we obtain

$$
\begin{aligned}
\sum_{i} \sum_{\alpha \in I_{i, \partial}}\left(\bar{n}_{\alpha}\right)^{2} & \leqslant C \sum_{i} \sum_{\alpha \in I_{i, \partial}} n_{\alpha}^{2} \leqslant C \sum_{i} \sum_{\alpha \in I_{i, \partial}}\left(n_{\alpha}-L^{d}\right)^{2} \leqslant C \sum_{i, \alpha} D\left(p_{\alpha}, L\right)^{2} \\
& \leqslant C \sum_{i, \alpha} \int_{\left(\mathcal{R}_{\alpha}\right)_{L}}|y|^{\gamma}\left|E_{\eta^{\prime}}\right|^{2} \leqslant C \int_{\left(\partial K_{R}^{\prime}\right)_{2 \cdot 3} \times[-\ell, \ell]^{k}}|y|^{\gamma}\left|E_{\eta^{\prime}}\right|^{2} \\
& \leqslant C M_{R, \varepsilon, \eta^{\prime}} \varepsilon^{-1} R^{d-1} .
\end{aligned}
$$

Finally, to control $m_{i}$, we write that, in view of (6.34)

$$
\left|m_{i}-1\right| \leqslant C \ell^{-d}\left(\int_{\partial D_{0} \cap \partial \tilde{H}_{i}}|y|^{\gamma}\left|E_{\eta}\right|\right)+2 \ell^{\gamma}\left|C_{0}\right|+\ell^{-d} \sum_{\alpha \in I_{i}} \bar{n}_{\alpha} .
$$

We note that the last term can be controlled by (6.44) using that $n_{\alpha} \leqslant n_{\alpha}^{2}$ since $n_{\alpha}$ is an integer, by $C M_{R, \varepsilon, \eta^{\prime}} \varepsilon^{-3} R^{-1}$. This can be made small if (6.2) holds, choosing $R_{0}$ large enough there. The second term (when it exists) can be bounded by $e_{\varepsilon, R}^{1 / 2} R^{\frac{\gamma-1}{2}} \varepsilon^{\gamma}$, which is small as soon as $R_{0}$ is chosen large enough in (6.2). The first term can be bounded by the Cauchy-Schwarz inequality and (6.28), first in the case when $k=0$ by

$$
C \ell^{-d} M_{R, \varepsilon, \eta}^{1 / 2} \varepsilon^{-1 / 2} R^{\frac{d-1}{2}} \ell^{\frac{d-1}{2}} \leqslant C M_{R, \varepsilon, \eta}^{1 / 2} \varepsilon^{-d-\frac{3}{2}} R^{-1}
$$

and in the case when $k=1$ by

$$
\ell^{-d} M_{R, \varepsilon, \eta}^{1 / 2} \varepsilon^{-1 / 2} R^{\frac{d-1}{2}} \ell^{\frac{d-1}{2}} \ell^{\frac{1+\gamma}{2}} \leqslant M_{R, \varepsilon, \eta}^{1 / 2} R^{\frac{\gamma-1}{2}} \varepsilon^{-\frac{1}{2}-d+\gamma} .
$$

These terms are all small as soon as (6.2) holds with $R_{0}$ chosen large enough.

Step 5. Estimating the energy of $\hat{E}_{\eta}$. 
For $l=1, \ldots, 4$, using the previous notation, we define $E_{l}:=\sum_{i} E_{i, l}$. We control successively the energy of each $E_{l}$. For $\alpha \in I_{i, \partial D}$, we use Lemma 6.6, and from the estimate (6.20) therein we obtain

$$
\int_{\tilde{R}_{\alpha}}|y|^{\gamma}\left|\nabla h_{1, \alpha}\right|^{2} \leqslant C(g(\eta)+1) n_{\alpha}^{2} .
$$

From (6.44) we thus obtain (absorbing the 1 into the $g(\eta)$ ) that the total contribution is

$$
\begin{aligned}
\int_{K_{R} \backslash K_{R}^{\prime} \times[-\ell, \ell]^{k}}|y|^{\gamma}\left|E_{1}\right|^{2} & =\sum_{i} \int_{\tilde{H}_{i}}|y|^{\gamma}\left|\nabla h_{i, 1}\right|^{2} \\
& \leqslant C M_{R, \varepsilon, \eta^{\prime}} g(\eta) \varepsilon^{-1} R^{d-1} \leqslant C M_{R, \varepsilon, \eta^{\prime}} g(\eta) \varepsilon R^{d},
\end{aligned}
$$

where for the last inequality we used (6.2).

For $E_{2}$, we use Lemma 6.4 , and control the number of $H_{i}$ by $C \varepsilon R^{d} \ell^{-d}$ to obtain, with (6.43),

$$
\int_{\left(K_{R} \backslash K_{R}^{\prime}\right) \times[-\ell, \ell]^{k}}|y|^{\gamma}\left|E_{2}\right|^{2} \leqslant C \varepsilon R^{d} \ell^{-d} C_{0}^{2} \ell^{\gamma+1+d} \leqslant C e_{\varepsilon, R} \varepsilon^{3} R^{d} .
$$

For $E_{3}$, we use (6.37) and Lemma 6.4 to get

$$
\begin{aligned}
\int|y|^{\gamma}\left|E_{3}\right|^{2} & \leqslant C \ell \sum_{i} \int_{\partial H_{i} \times[-\ell, \ell]^{k}}|y|^{\gamma} g_{i}^{2} \\
& \leqslant C \ell \int_{\partial K_{R}^{\prime} \times[-\ell, \ell]^{k}}|y|^{\gamma}\left|E_{\eta}\right|^{2}+C \ell \sum_{i, \alpha \in I_{i}}\left(\bar{n}_{\alpha}\right)^{2} \\
& \leqslant C \ell\left(M_{R, \varepsilon, \eta}+M_{R, \varepsilon, \eta^{\prime}}\right) \varepsilon^{-1} R^{d-1} \leqslant C\left(M_{R, \varepsilon, \eta}+M_{R, \varepsilon, \eta^{\prime}}\right) \varepsilon R^{d},
\end{aligned}
$$

where we have used (6.28), (6.44), and the geometric properties of $\tilde{\mathcal{R}}_{\alpha}$.

For $E_{4}$, we use Lemma 6.5 , and multiply by the number of $\mathcal{R}_{\alpha}$, which is proportional to the volume of the region, and hence bounded by $C \varepsilon R^{d}$, to obtain

$$
\int_{\tilde{K}_{R}}|y|^{\gamma}\left|E_{4}\right|^{2}=\sum_{i, \alpha \in I_{i}} \int_{\tilde{R}_{\alpha}}|y|^{\gamma}\left|\nabla h_{4, \alpha}\right|^{2} \leqslant C g(\eta) \varepsilon R^{d}
$$

Finally, in $D_{1}$, we use the obvious analog of Lemma 6.4 to obtain

$$
\begin{aligned}
\int_{D_{1}}|y|^{\gamma}\left|\hat{E}_{\eta}\right|^{2} & \leqslant C R \int_{\partial D_{1}}|y|^{\gamma}|\phi|^{2} \leqslant C R\left(\int_{\partial D_{0} \cap \partial D_{1}}|y|^{\gamma}\left|E_{\eta} \cdot v\right|^{2}+C_{0}^{2} \ell^{\gamma}\left(\left|K_{R}\right|-\left|K_{R}^{\prime}\right|\right)\right) \\
& \leqslant C e_{\varepsilon, R} \varepsilon^{2} R^{d}+C e_{\varepsilon, R} \varepsilon R^{d} \leqslant C e_{\varepsilon, R} \varepsilon R^{d},
\end{aligned}
$$

where we used (6.30) and (6.43).

Combining (6.47)-(6.51), we conclude that

$$
\int_{K_{R} \times \mathbb{R}^{k}}|y|^{\gamma}\left|\hat{E}_{\eta}\right|^{2} \leqslant \int_{K_{R}^{\prime} \times \mathbb{R}^{k}}|y|^{\gamma}\left|E_{\eta}\right|^{2}+C\left(\left(M_{R, \varepsilon, \eta^{\prime}}+1\right) g(\eta) \varepsilon R^{d}+\left(M_{R, \varepsilon, \eta}+e_{\varepsilon, R}\right) \varepsilon R^{d}\right),
$$

and since it is clear by construction that $\hat{E}_{\eta}=\Phi_{\eta}(\hat{E})$, the desired result holds. This concludes the proof of Proposition 6.1. 
Remark 6.7. It follows from the discussion in the proof that, if the points in $\hat{\Lambda} \cap\left(K_{R} \backslash K_{R}^{\prime}\right)$ are each displaced by a distance $\leqslant \eta_{0} / 4$ (with $\eta_{0}$ as in the statement of the proposition), there exists a vector field $\hat{E}$ compatible with the modified configuration and satisfying the exact same conclusions. Indeed one may displace the barycenters $p_{\alpha}$ of the $\tilde{\mathcal{R}}_{\alpha}$ in the proof by a quarter of their minimal distance without changing the conclusions.

\section{The upper bound on the energy}

We are now in a position to use Proposition 6.1 to construct a test configuration approximating $\min H_{n}$.

We will need the following result along the lines of [49, Lemma 5.4].

Lemma 7.1. Assume that we are in one of the cases for which $k=1$. There exists $E, a$ minimizer of $\mathcal{W}_{\eta}$ over $\mathcal{A}_{1}$, which satisfies

$$
\lim _{z \rightarrow \infty} \lim _{R \rightarrow \infty} \frac{1}{R^{d}} \int_{K_{R} \times(\mathbb{R} \backslash(-z, z))}|y|^{\gamma}|E|^{2}=0 .
$$

Proof. We claim that there exists an $\mathbb{R}^{d}$-translation-invariant probability measure on vector fields $P$ which is concentrated on minimizers of $\mathcal{A}_{1}$. Assuming this, the result follows by applying the multiparameter ergodic theorem as in [4] on the cubes $K_{R}=$ $[-R / 2, R / 2]^{d}$ to the function $f_{z}(E)=\int_{[-1,1] \times\{|y|>z\}}|y|^{\gamma}|E|^{2}$ with $z>1$. Indeed, the multiparameter ergodic theorem yields that $\int f_{z}(E) d P(E)=\int f_{z}^{*}(E) d P(E)$ with

$$
f_{z}^{*}(E):=\lim _{R \rightarrow \infty} \frac{1}{\left|K_{R}\right|} \int_{K_{R}} f(E(x+\cdot)) d x,
$$

and we may check that

$$
f_{z}^{*}(E) \geqslant C \lim _{R \rightarrow \infty} \frac{1}{R^{d}} \int_{K_{R} \times\{|y|>z\}}|y|^{\gamma}|E|^{2} .
$$

But, for any $E \in \mathcal{A}_{1}$, the family of function $\left\{f_{z}(E)\right\}_{z>1}$ decreases to 0 as $z$ increases to $+\infty$ and is dominated by $f_{1}$; thus, by dominated convergence, we have $\lim _{z \rightarrow 0} \int f_{z}(E) d P(E)=$ 0. Combining this with the above, it follows that

$$
\lim _{z \rightarrow \infty} \int\left(\lim _{R \rightarrow \infty} \frac{1}{R^{d}} \int_{K_{R} \times\{|y|>z\}}|y|^{\gamma}|E|^{2}\right) d P(E)=0,
$$

and by Fatou's lemma, for $P$-a.e. $E$ we must have

$$
\lim _{z \rightarrow \infty} \lim _{R \rightarrow \infty} \frac{1}{R^{d}} \int_{K_{R} \times\{|y|>z\}}|y|^{\gamma}|E|^{2}=0 .
$$

The result follows, since $P$ is concentrated on minimizers of $\mathcal{W}_{\eta}$ on $\mathcal{A}_{1}$.

For the existence of $P$, we start from some $E$ minimizing $\mathcal{W}_{\eta}$ over $\mathcal{A}_{1}$. The existence of such an $E$ can be proven exactly as in [44, Appendix]. We let $K_{n}$ be the cubes $[-n / 2, n / 2]^{d}$ 
in $\mathbb{R}^{d} \times\{0\}$, and $P_{n}$ be the push-forward of the normalized Lebesgue measure on $K_{n}$ by the map $x \mapsto E((x, 0)+\cdot)$. We also let, as in $\S 5$,

$$
\mathbf{f}_{n, \eta}(\mathcal{Y})=\int_{\mathbb{R}^{d+1}} \chi|y|^{\gamma}|\mathcal{Y}|^{2}
$$

if $\mathcal{Y}(X)=E_{\eta}(X+(x, 0))$ for some $x \in K_{n}$, and $+\infty$ otherwise. Then the exact same arguments as in $\S 5$ apply to this setting (the only difference is that we have from the beginning an infinite number of points); in particular, we have the obvious compactness result analogous to Lemma 5.1. This ensures that the abstract result [47, Theorem 3] can be applied, and it yields at the end

$$
\liminf _{n \rightarrow \infty} \frac{1}{\left|K_{n}\right|} \int_{K_{n} \times \mathbb{R}}|y|^{\gamma}\left|E_{\eta}\right|^{2} \geqslant \int \mathcal{W}_{\eta}(E) d P(E),
$$

with $P$ an $\mathbb{R}^{d}$-translation-invariant probability measure. The left-hand side in this relation is $\mathcal{W}_{\eta}(E)=\min \mathcal{W}_{\eta}$ by definition. It follows that $P$ is concentrated on minimizers of $\mathcal{W}_{\eta}$.

Remark 7.2. With the same reasoning and Fatou's lemma, we could prove the same for a minimizer of $\mathcal{W}$ itself.

This proposition implies that we can find a minimizer $E$ of $\mathcal{W}_{\eta}$ over $\mathcal{A}_{1}$ for which, given $\varepsilon$, we have $e_{\varepsilon, R} \leqslant 1$ as soon as $R$ is large enough (in terms of $\varepsilon$ ). We may now conclude the proof of Proposition 1.4. For the existence of minimizers of $\mathcal{W}$ and $\mathcal{W}_{\eta}$ over $\mathcal{A}_{1}$, as mentioned, we may argue as in [44, Appendix]. The existence of sequences of periodic minimizers then follows exactly as in $[44,48]$ (so we do not give all the details): we take a minimizer of $\mathcal{W}_{\eta}$ over $\mathcal{A}_{1}$ satisfying the results of Lemma 7.1, apply Proposition 6.1 to it with some $R$ large enough, then multisymmetrize it by reflexion and periodize the result to obtain some periodic $E_{R, \eta}$ with almost the same energy, i.e. given $\varepsilon>0$, if $R$ is large enough depending on $\eta$ and $\varepsilon$, we have $\mathcal{W}_{\eta}\left(E_{R, \eta}\right) \leqslant \min _{\mathcal{A}_{1}} \mathcal{W}_{\eta}+\varepsilon$. This implies that there is a periodic minimizing sequence for $\min \mathcal{W}_{\eta}$ over $\mathcal{A}_{1}$. Taking a diagonal sequence $\eta \rightarrow 0, R \rightarrow \infty$, we may also conclude that there exists a periodic minimizing sequence for $\mathcal{W}$, and this finishes the proof of Proposition 1.4.

We are now going to prove the matching upper bound corresponding to the lower bound given in Proposition 5.2.

Proposition 7.3. Assume that (1.8)-(1.10) and (1.12)-(1.14) hold. For any $\varepsilon>0$, there exists $r>0$ such that for $n \in \mathbb{N}^{*}$ there exists $A_{n} \subset\left(\mathbb{R}^{d}\right)^{n}$ with $\left|A_{n}\right| \geqslant n !\left(\frac{\pi r^{d}}{n}\right)^{n}$ such that for all $\left(x_{1}, \ldots, x_{n}\right) \in A_{n}$ it holds that

$$
\limsup _{n \rightarrow \infty} n^{-1-\frac{s}{d}}\left(H_{n}\left(x_{1}, \ldots, x_{n}\right)-n^{2} \mathcal{E}\left(\mu_{V}\right)\right) \leqslant \min \tilde{\mathcal{W}}+\varepsilon
$$

in case (1.2), respectively

$$
\limsup _{n \rightarrow \infty} n^{-1}\left(H_{n}\left(x_{1}, \ldots, x_{n}\right)-n^{2} \mathcal{E}\left(\mu_{V}\right)+\frac{n}{d} \log n\right) \leqslant \min \tilde{\mathcal{W}}+\varepsilon
$$

in cases (1.3)-(1.4). 
The proof follows precisely the strategy of $[44, \S 6]$, with some care to be taken about the extra dimension introduced in our case. The main difficulty comes from the possible degeneracy of $\mu_{V}$ near $\partial \Sigma$ that we allow, as in [49]. Indeed, we need to partition $\Sigma^{\prime}=$ $n^{1 / d} \Sigma$ into nondegenerate regions in which $\int \mu_{V}^{\prime} \in \mathbb{N}$, in which we can paste a screened minimizer of $\mathcal{W}$ provided by Proposition 6.1 . When $\mu_{V}^{\prime}$ becomes very small these regions may have to become very large, or, worse, very elongated in some direction, and this would prevent the application of Proposition 6.1. As in [49], this is overcome by allowing an exceptional narrow boundary layer in which the construction is less optimal but induces only negligible errors.

Proof. Step 1. Subdividing the domain.

For $t>0$, we define the tubular neighborhood of $\partial \Sigma^{\prime}$ and its boundary to be

$$
\Sigma_{t}^{\prime}=\left\{x \in \Sigma^{\prime}, \operatorname{dist}\left(x, \partial \Sigma^{\prime}\right)<t\right\} \quad \Gamma_{t}=\left\{x \in \Sigma^{\prime}, \operatorname{dist}\left(x, \partial \Sigma^{\prime}\right)=t\right\} .
$$

Since (1.12) holds, $\Gamma_{t}$ is $C^{1}$ for $t<t_{c}$ small enough.

Pick $1>\underline{m}>0$ a small number. By assumption (1.14), if $\alpha>0$ in that assumption,

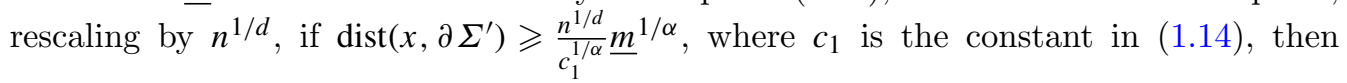
$\mu_{V}^{\prime}(x) \geqslant \underline{m}$, while if $\operatorname{dist}\left(x, \partial \Sigma^{\prime}\right) \leqslant \frac{n^{1 / d}}{c_{1}} \underline{m}^{1 / \alpha}$ we have $\mu_{V}^{\prime}(x) \leqslant \frac{c_{2}}{c_{1}} \underline{m}$. We may even find

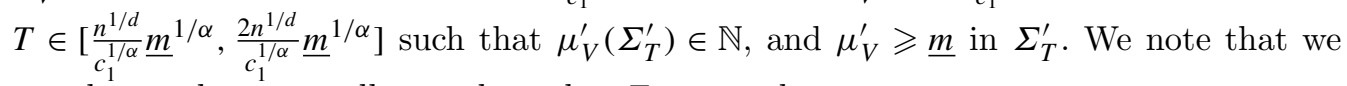
may have taken $\underline{m}$ small enough so that $T<t_{c}$ and

$$
\frac{1}{2} \mathcal{H}^{d-1}\left(\partial \Sigma^{\prime}\right) \leqslant \mathcal{H}^{d-1}\left(\partial \Sigma_{t}^{\prime}\right) \leqslant 2 \mathcal{H}^{d-1}\left(\partial \Sigma^{\prime}\right) \text { for all } t \leqslant T .
$$

If $\alpha=0$ in assumption (1.14), then $\mu_{V}^{\prime}$ is bounded below by a positive constant on its support, and we simply take $T=0$. For brevity, in what follows we denote $\mathcal{H}^{d-1}\left(\partial \Sigma^{\prime}\right)$ by $\left|\partial \Sigma^{\prime}\right|$.

Step 2. Defining a vector field in $\Sigma_{T}^{\prime}$.

In the region $\Sigma_{T}^{\prime}$, we have the lower bound $\mu_{V} \geqslant \underline{m}$, and there is no degeneracy. We may then proceed as in $[44,48]$. We start by subdividing $\Sigma_{T}^{\prime}$ into hyper-rectangles of size comparable to $R$ for $R$ large enough, then producing a rescaled version of the construction of Proposition 6.1 on each such hyper-rectangle. We make sure the points all remain well separated so as to ensure that we stay in the equality case in Lemma 2.3 and in formula (5.1).

The next lemma is a straightforward modification of [48, Lemma 6.5].

Lemma 7.4 (Tiling the interior of the domain). There exists a constant $C_{0}>0$ depending on $\underline{m}$ and $\bar{m}$ such that, given any $R>1$, there exists for any $n \in \mathbb{N}^{*}$ a collection $\mathcal{K}_{n}$ of closed hyperrectangles in $\Sigma_{T}^{\prime}$ with disjoint interiors, whose sidelengths are between $2 R$ and $2\left(R+C_{0} / R\right)$, and which are such that

$$
\left\{x \in \Sigma_{T}^{\prime}: d\left(x, \partial \Sigma_{T}^{\prime}\right) \geqslant C_{0} R\right\} \subset \bigcup_{K \in \mathcal{K}_{n}} K:=\Sigma_{\text {int }}^{\prime},
$$




$$
\bigcup_{K \in \mathcal{K}_{n}} K \subset\left\{x \in \Sigma^{\prime}: d\left(x, \partial \Sigma^{\prime}\right) \geqslant \frac{1}{2} C_{0} R\right\},
$$

and

$$
\forall K \in \mathcal{K}_{n}, \quad \int_{K} \mu_{V}^{\prime} \in \mathbb{N} .
$$

For each $K \in \mathcal{K}_{n}$ as above, we denote

$$
m_{K}:=f_{K} \mu_{V}^{\prime},
$$

and note that $m_{K}|K| \in \mathbb{N}$. By Proposition 1.4, given $\varepsilon>0$ as in the statement of Proposition 7.3 , we may find a periodic $E$ in $\mathcal{A}_{1}$ such that $\mathcal{W}(E) \leqslant \min _{\mathcal{A}_{1}} \mathcal{W}+\frac{\varepsilon}{4 \bar{m}^{s / d}}$. If $k=1$, we note that, by the periodicity of $E$, we must have

$$
\lim _{z \rightarrow \infty} \lim _{R \rightarrow \infty} \frac{1}{R^{d}} \int_{K_{R} \times(\mathbb{R} \backslash(-z, z))}|y|^{\gamma}|E|^{2}=0,
$$

and hence the assumptions of Proposition 6.1 are satisfied for $E$ if $R$ is large enough. Also, by Proposition 4.3, the points in $E$ are well separated, and the screening construction preserves that property, and makes the points well separated from the boundary. By Proposition 2.4 we have

$$
\mathcal{W}_{\eta}(E) \leqslant \mathcal{W}(E)+C \eta^{\frac{d-s}{2}}
$$

For each $K \in \mathcal{K}_{n}$ we thus apply Proposition 6.1 to $E$ with $R m_{K}^{1 / d}$ and over the hyperrectangle $m_{K}^{1 / d} K$. We may do this since $m_{K}|K|=\int_{K} \mu_{V}^{\prime}$ is an integer by (7.6), and its sidelengths are indeed in $\left[2 R m_{K}^{1 / d}, 3 R m_{K}^{1 / d}\right]$ if $R$ is chosen large enough depending on $C_{0}$. We call the result of this screening $\hat{E}$, and we define $E_{K}$ to be

$$
E_{K}=\nabla h_{K}+\sigma_{m_{K}} \hat{E} \quad \text { on } K \times \mathbb{R}^{k}
$$

where $\sigma_{m}$ denotes the rescaling of a vector field to scale $m$ as follows:

$$
\sigma_{m} E=m^{\frac{s+1}{d}} E\left(m^{1 / d} \cdot\right),
$$

and $h_{K}$ is introduced to correct for the difference between $m_{K}$ and $\mu_{V}^{\prime}$ as the solution to

$$
\begin{cases}-\operatorname{div}\left(|y|^{\gamma} \nabla h_{K}\right)=c_{d, s}\left(m_{K}-\mu_{V}^{\prime} \delta_{\mathbb{R}^{d}}\right) & \text { in } K \times[-1,1]^{k}, \\ \partial_{\nu} h_{K}=0 & \text { on } \partial\left(K \times[-1,1]^{k}\right) .\end{cases}
$$

By Lemma 6.4, we have

$$
\left\|\nabla h_{K}\right\|_{L^{2}\left(K \times \mathbb{R}^{k},|y|^{\gamma}\right)} \leqslant C_{R}\left\|m_{K}-\mu_{V}^{\prime}\right\|_{L^{\infty}\left(K \times \mathbb{R}^{k}\right)} \leqslant C_{R} n^{-\beta / d},
$$

using assumption (1.13).

The fact that $E$ has been chosen to be an almost minimizer of $\mathcal{W}$ over $\mathcal{A}_{1},(7.8)$, and the conclusions of Proposition 6.1 and (7.9) yield 


$$
\begin{aligned}
& \int_{m_{K}^{1 / d} K \times \mathbb{R}^{k}}|y|^{\gamma}\left|\hat{E}_{m_{K}^{1 / d} \eta}\right|^{2}-m_{K}|K| c_{d, s} g\left(m_{K}^{1 / d} \eta\right) \\
& \leqslant m_{K}|K|\left(\min _{\mathcal{A}_{1}} \mathcal{W}+C\left(m_{K}^{1 / d} \eta\right)^{\frac{d-s}{2}}+\frac{\varepsilon}{4 \bar{m}^{1+s / d}}+C_{\eta} o_{R}(1)\right),
\end{aligned}
$$

where $o_{R}(1)$ tends to 0 as $R \rightarrow \infty$. By change of scales, we have

$$
\int_{K \times \mathbb{R}^{k}}|y|^{\gamma}\left|\left(\sigma_{m_{K}} \hat{E}\right)_{\eta}\right|^{2}=m_{K}^{\frac{s}{d}} \int_{m_{K}^{1 / d} K \times \mathbb{R}^{k}}|y|^{\gamma}\left|\hat{E}_{m_{K}^{1 / d} \eta}\right|^{2},
$$

with the convention that $s=0$ in cases (1.3)-(1.4).

The scaling of $\mathcal{W}(1.35)-(1.36)$, together with (7.11) and (7.12), thus yields

$$
\begin{aligned}
\int_{K \times \mathbb{R}}|y|^{\gamma}\left|\left(E_{K}\right)_{\eta}\right|^{2} \leqslant & m_{K}|K| c_{d, s} g(\eta) \\
& +|K|\left(\min _{\mathcal{A}_{m_{K}}} \mathcal{W}+m_{K}^{1+s / d} \frac{\varepsilon}{4 \bar{m}^{s / d}}+C m_{K}^{1+\frac{s}{d}+\frac{1}{2}-\frac{s}{2 d}} \eta^{\frac{d-s}{2}}+C_{\eta} o_{R}(1)\right) .
\end{aligned}
$$

The interior electric field is then set to be $E_{\text {int }}=\sum_{K \in \mathcal{K}_{n}} E_{K} \mathbb{1}_{K \times \mathbb{R}}$, and it satisfies

$$
-\operatorname{div}\left(|y|^{\gamma} E_{\text {int }}\right)=c_{d, s}\left(\sum_{p \in \Lambda_{\text {int }}} \delta_{p}-\mu_{V}^{\prime} \mathbb{1}_{\Sigma_{\text {int }}^{\prime}} \delta_{\mathbb{R}^{d}}\right) \quad \text { in } \mathbb{R}^{d+k},
$$

for some discrete set $\Lambda_{\text {int }}$, made of well-separated points (i.e. points whose distance to each other is bounded below by a constant independent of $R$ and $\eta$ ). Indeed, no divergence is created at the interfaces between the hyperrectangles since the normal components of $\nabla h_{K}$ and $\hat{E}$ are zero.

Step 2. Placing the points in $\Sigma^{\prime} \backslash \Sigma_{\text {int }}^{\prime}$.

Since $\partial \Sigma_{T}^{\prime} \in C^{1}$ as seen in the first step, the set

$$
\Sigma_{\text {bound }}^{\prime}:=\Sigma_{T}^{\prime} \backslash \Sigma_{\text {int }}^{\prime}
$$

is a strip near $\partial \Sigma_{T}^{\prime}$ of volume $\leqslant C n^{\frac{d-1}{d}}$ and width $\geqslant \frac{1}{2} C_{0} R$ by Lemma 7.4. Since $\int_{\Sigma_{T}^{\prime}} \mu_{V}^{\prime} \in$ $\mathbb{N}$ and (7.6) holds, $\int_{\Sigma_{\text {bound }}^{\prime}} \mu_{V}^{\prime}$ is also an integer. We now need to place $\int_{\Sigma_{\text {bound }}^{\prime}} \mu_{V}^{\prime}$ points in $\Sigma_{\text {bound }}^{\prime}$, all separated by distances bounded below by some constant $r_{0}>0$ independent of $n, \eta$, and $R$ (up to changing $r_{0}$ if necessary). Proceeding as in [48, $\S 6.3$, Step 4], using the fact that $\partial \Sigma_{T}^{\prime}$ is $C^{1}$, and making several layers, we may split $\Sigma_{\text {bound }}^{\prime}$ into regions $\mathcal{C}_{i}$ such that $\int_{\mathcal{C}_{i}} \mu_{V}^{\prime}=1$ and $\mathcal{C}_{i}$ is a set with piecewise $C^{1}$ boundary, containing a ball of radius $C_{1}$ and contained in a ball $B_{i}$ of radius $C_{2}$, where $C_{1}, C_{2}>0$ depend only on the dimension.

We continue with examining $\Sigma^{\prime} \backslash \Sigma_{T}^{\prime}$ in the case when $T \neq 0$ (i.e. if $\alpha>0$ in (1.14)). For $\Sigma_{t}^{\prime}$ as defined in Step 1, let us set

$$
f(t)=\int_{\Sigma_{t}^{\prime}} \mu_{V}^{\prime}(x) d x
$$


In view of assumption (1.14) we have

$$
f(t) \geqslant c_{1} \int_{0}^{t} \mathcal{H}^{d-1}\left(\Gamma_{s}\right)\left(\frac{s}{n^{1 / d}}\right)^{\alpha} d s .
$$

In view of (7.3) and the scaling, we deduce that

$$
f(t) \geqslant \frac{c_{1}|\partial \Sigma|}{2(\alpha+1)}\left(t^{\alpha+1}\right) n^{\frac{d-1-\alpha}{d}} .
$$

We now define inductively a sequence of $t$ terminating at $T$ : let $t_{0}=0$, and let $t_{1}$ be the smallest such that $f\left(t_{1}\right) \in \mathbb{N}$ and

$$
t_{1} \geqslant n^{\frac{\alpha}{d(\alpha+d)}} \quad f\left(t_{1}\right) \geqslant\left|\partial \Sigma^{\prime}\right| n^{\frac{\alpha(1-d)}{d(\alpha+d)}}=|\partial \Sigma| n^{\frac{d-1}{d}\left(1-\frac{\alpha}{\alpha+d}\right)} .
$$

In view of (7.17), we may satisfy this condition with $t_{1} \leqslant C n^{\frac{\alpha}{d(\alpha+d)}}$, where $C$ depends only on $c_{1}$ and $\alpha$. We let $N_{1}=f\left(t_{1}\right)$, and note that from the above discussion we have

$$
\left|\partial \Sigma^{\prime}\right| n^{\frac{\alpha(1-d)}{d(\alpha+d)}} \leqslant N_{1} \leqslant C\left|\partial \Sigma^{\prime}\right| n^{\frac{\alpha(1-d)}{d(\alpha+d)}} .
$$

We write

$$
n^{\frac{\alpha}{d(\alpha+d)}} \leqslant t_{1}-t_{0} \leqslant C n^{\frac{\alpha}{d(\alpha+d)}} .
$$

Let $j \geqslant 2$. Given $t_{j-1}$, we then let $t_{j}$ be the smallest such that

$$
t_{j} \geqslant t_{j-1}+n^{\frac{\alpha}{d^{2}}} t_{j-1}^{-\frac{\alpha}{d}} \quad f\left(t_{j}\right)-f\left(t_{j-1}\right) \in \mathbb{N},
$$

and we define $N_{j}=f\left(t_{j}\right)-f\left(t_{j-1}\right)$. Since $\mu_{V}^{\prime}$ is bounded below in $\left(\Sigma_{t_{j-1}}^{\prime}\right)^{c}$ by $c_{1} t_{j-1}^{\alpha} n^{-\frac{\alpha}{d}}$ in view of (1.14), we deduce that

$$
\begin{aligned}
N_{j} & \geqslant \frac{1}{2} c_{1}\left|\partial \Sigma^{\prime}\right|\left(t_{j}-t_{j-1}\right)\left(t_{j-1} n^{-1 / d}\right)^{\alpha} \geqslant \frac{1}{2} c_{1}|\partial \Sigma| n^{\frac{d-1}{d}} t_{j-1}^{\alpha} n^{-\frac{\alpha}{d}} n^{\frac{\alpha}{d^{2}}} t_{j-1}^{-\frac{\alpha}{d}} \\
& =\frac{c_{1}}{2}|\partial \Sigma| n^{\frac{d^{2}-d-\alpha d+\alpha}{d^{2}}} t_{j-1}^{\frac{\alpha(d-1)}{d}} \geqslant \frac{c_{1}}{2}|\partial \Sigma| n^{\frac{d-1}{d}\left(\alpha^{2} d-\alpha+d\right)},
\end{aligned}
$$

where we have inserted that $t_{j-1} \geqslant t_{1} \geqslant n^{\frac{\alpha}{d(\alpha+d)}}$. The exponent in the right-hand side is positive, and so we conclude that, as soon as $n$ is large enough, we may make $N_{j} \geqslant 1$ while keeping

$$
t_{j}-t_{j-1} \leqslant 2 n^{\frac{\alpha}{d^{2}}} t_{j-1}^{-\frac{\alpha}{d}}
$$

We thus write, for all $j \geqslant 2$,

$$
n^{\frac{\alpha}{d^{2}}} t_{j-1}^{-\frac{\alpha}{d}} \leqslant t_{j}-t_{j-1} \leqslant 2 n^{\frac{\alpha}{d^{2}}} t_{j-1}^{-\frac{\alpha}{d}},
$$

and we note that, since $t_{j-1} \geqslant t_{1} \geqslant n^{\frac{\alpha}{d(\alpha+d)}}$, we have $\frac{t_{j}-t_{j-1}}{t_{j-1}} \leqslant 2 n^{\frac{\alpha}{d^{2}}} n^{\left(-\frac{\alpha}{d}-1\right) \frac{\alpha}{d(\alpha+d)}}=2$, and hence

$$
t_{j-1} \leqslant t_{j} \leqslant 3 t_{j-1}
$$

To bound $t_{j}-t_{j-1}$ from below, we write $t_{j}-t_{j-1} \geqslant n^{\frac{\alpha}{d^{2}}} T^{-\alpha / d}=2^{-\alpha / d} c_{1}^{1 / d} \underline{m}^{-d}$, and we may write

$$
2^{-\alpha / d} c_{1}^{1 / d} \leqslant t_{j}-t_{j-1} \leqslant 2 n^{\frac{\alpha}{d(\alpha+d)}} .
$$


(7.22) implies, in view of (1.14), that

$$
c_{1} n^{-\alpha / d} t_{j-1}^{\alpha} \leqslant \mu_{V}^{\prime} \leqslant 3^{\alpha} c_{2} n^{-\alpha / d} t_{j-1}^{\alpha} \quad \text { in } \Sigma_{t_{j}}^{\prime} \backslash \Sigma_{t_{j-1}}^{\prime},
$$

or, in other words, using (7.21), that

$$
C\left(t_{j}-t_{j-1}\right)^{-d} \leqslant \mu_{V}^{\prime} \leqslant C^{\prime}\left(t_{j}-t_{j-1}\right)^{-d} \quad \text { in } \Sigma_{t_{j}}^{\prime} \backslash \Sigma_{t_{j-1}}^{\prime}
$$

for some positive $C, C^{\prime}$ depending only on the previous constants.

We may also bound $N_{j}$ from above, using the upper bound in (1.14) and (7.3):

$$
N_{j}=f\left(t_{j}\right)-f\left(t_{j-1}\right) \leqslant 2 c_{2}\left(t_{j}-t_{j-1}\right)\left|\partial \Sigma^{\prime}\right| n^{-\frac{\alpha}{d}} t_{j}^{\alpha},
$$

and combining this with (7.20) and (7.22) we deduce that

$$
\frac{1}{2} c_{1}\left|\partial \Sigma^{\prime}\right|\left(t_{j}-t_{j-1}\right) t_{j-1}^{\alpha} n^{-\alpha / d} \leqslant N_{j} \leqslant 6 c_{2}\left|\partial \Sigma^{\prime}\right|\left(t_{j}-t_{j-1}\right) t_{j-1}^{\alpha} n^{-\alpha / d} .
$$

This construction terminates at $t_{J}=T$, and if (7.21) is not satisfied at $j=J$ we may always merge the last two steps to have it satisfied up to a factor 2 in the right-hand side.

The next step consists, for each $1 \leqslant j \leqslant J$, in partitioning $\Sigma_{t_{j}}^{\prime} \backslash \Sigma_{t_{j-1}^{\prime}}$ into $N_{j}$ regions of sidelength comparable to $t_{j}-t_{j-1}$, in such a way that $\int_{\mathcal{C}_{i}} \mu_{V}=1$ in each region. To do that, we partition $\Gamma_{t_{j-1}}$ into $N_{j}$ regions, and we deduce a partitioning of $\Sigma_{t_{j}}^{\prime} \backslash \Sigma_{t_{j-1}^{\prime}}$ by going along the normals to $\Gamma_{t_{j-1}}$ on the boundaries of the partition of $\Gamma_{t_{j-1}}$. Given a cell $\tilde{\mathcal{C}}$ on $\Gamma_{t_{j-1}}$, we denote by $\mathcal{C}$ the corresponding cell in $\Sigma_{t_{j}}^{\prime} \backslash \Sigma_{t_{j-1}^{\prime}}$ obtained this way. We claim that we can construct $N_{j}$ such cells $\tilde{\mathcal{C}}_{i}$ such that $\int_{\mathcal{C}_{i}} \mu_{V}=1$ for each $i$ and the sidelengths of the cells are comparable to $t_{j}-t_{j-1}$. First, we note that, if $j \geqslant 2$, from (7.3), (7.26), and (7.21) we have that $\left|\Gamma_{t_{j-1}}\right| / N_{j}$ is bounded above and below by constants times $\left(t_{j}-t_{j-1}\right)^{d-1}$. If $j=1$, the same holds using instead (7.19) and (7.18). We may thus subdivide $\Gamma_{t_{j-1}}$ iteratively, the same way we did for Lemma 7.4, or above as in $[48, \S 7.3$, Step 4], except we are on a curved hypersurface instead of a flat one. In order to ensure that the result can be achieved, we need a lower and upper bound on the density of $\mu_{V}^{\prime}$ by a constant times $\left(t_{j}-t_{j-1}\right)^{-d}$, provided precisely by (7.25) in the case when $j \geqslant 2$. In the case when $j=1$, then we replace the lower bound by a bound on the integrated density of $\mu_{V}^{\prime}$ along the normal to $\partial \Gamma_{0}=\partial \Sigma^{\prime}$ : for $x \in \partial \Sigma^{\prime}$ and $\vec{v}$ the inner normal at $x$, we have

$$
\int_{0}^{t_{1}} \mu_{V}^{\prime}(x+s \vec{v}) d s \geqslant c_{1} \int_{0}^{n^{\frac{\alpha}{d(\alpha+d)}}}\left(\frac{s}{n^{1 / d}}\right)^{\alpha} d s=\frac{c_{1}}{1+\alpha} n^{-\alpha / d} n^{\frac{\alpha(\alpha+1)}{d(\alpha+d)}}=n^{\frac{\alpha(1-d)}{d(\alpha+1)}},
$$

which is comparable to $t_{1}^{d-1}$, as desired.

Thus we may indeed partition $\Sigma_{t_{j}}^{\prime} \backslash \Sigma_{t_{j-1}^{\prime}}$ into $N_{j}$ cells $\mathcal{C}_{i}$, which are contained in a ball $B_{i}$ of radius $C_{2}\left(t_{j}-t_{j-1}\right)$ and contain a ball of radius $C_{1}\left(t_{j}-t_{j-1}\right)$, for some $C_{1}, C_{2}$ depending only on the above constants $c_{1}, c_{2}, \alpha, d$, and in which $\int_{\mathcal{C}_{i}} \mu_{V}^{\prime}=1$. We may add the cells obtained this way, for $j$ ranging from 0 to $J$, to the ones obtained at the beginning of the step in $\Sigma_{\text {bound }}^{\prime}$. We call $r_{i}$ their diameter scale, i.e. $r_{i}=1$ in the case of 
the cells obtained in $\Sigma_{\text {bound }}^{\prime}$, and $r_{i}=t_{j}-t_{j-1}$ for the cells in $\Sigma_{t_{j}}^{\prime} \backslash \Sigma_{t_{j-1}}^{\prime}$. From (7.23) we have $0<c \leqslant r_{i} \leqslant 2 n^{\frac{\alpha}{d(\alpha+d)}}$. We let $p_{i}$ be the center of each inner ball included in $\mathcal{C}_{i}$. This way $p_{i}$ is at distance $\geqslant c r_{i}$ from $\partial \mathcal{C}_{i}$, and thus all the points are separated by distances independent of $R, \eta$, and $n$.

Step 3. Completing the construction.

We then let $v_{i}$ solve

$$
\begin{cases}-\operatorname{div}\left(|y|^{\gamma} \nabla v_{i}\right)=c_{d, s}\left(\delta_{p_{i}}-\mu_{V}^{\prime} \mathbb{1}_{\mathcal{C}_{i}} \delta_{\mathbb{R}^{d}}\right) & \text { in } B_{i} \times\left[-r_{i}, r_{i}\right]^{k}, \\ \nabla v_{i} \cdot \vec{v}=0 & \text { on } \partial B_{i} \times\left[-r_{i}, r_{i}\right]^{k},\end{cases}
$$

and we set

$$
E_{\mathrm{bound}}:=\sum_{i} \mathbb{1}_{\mathcal{C}_{i}} \nabla v_{i}
$$

This way, we globally have

$$
-\operatorname{div}\left(|y|^{\gamma} E_{\text {bound }}\right)=c_{d, s}\left(\sum_{i} \delta_{p_{i}}-\mu_{V}^{\prime} \mathbb{1}_{\mathcal{C}_{i}}\right) \quad \text { in } \mathbb{R}^{d+k}
$$

We now evaluate the energy generated by these vector fields. For each cell $i$, we write $v_{i}=h_{i}+u_{i}$, where

$$
-\operatorname{div}\left(|y|^{\gamma} \nabla h_{i}\right)=c_{d, s}\left(\delta_{p_{i}}-\left(f_{\mathcal{C}_{i}} \mu_{V}^{\prime} \delta_{\mathbb{R}^{d}}\right) \mathbb{1}_{\mathcal{C}_{i}} \delta_{\mathbb{R}^{d}}\right) \quad \text { in } B_{i} \times\left[-r_{i}, r_{i}\right]^{k}
$$

and

$$
-\operatorname{div}\left(|y|^{\gamma} \nabla u_{i}\right)=c_{d, s}\left(\left(f_{\mathcal{C}_{i}} \mu_{V}^{\prime} \delta_{\mathbb{R}^{d}}\right)-\mu_{V}^{\prime}\right) \mathbb{1}_{\mathcal{C}_{i}} \delta_{\mathbb{R}^{d}} \quad \text { in } B_{i} \times\left[-r_{i}, r_{i}\right]^{k}
$$

with the same zero Neumann boundary data. The energy of $u_{i}$ is estimated using assumption (1.13) and $r_{i} \leqslant 2 n^{\frac{\alpha}{d(\alpha+d)}}$ :

$$
\int_{B_{i} \times\left[-r_{i}, r_{i}\right]^{k}}|y|^{\gamma}\left|\nabla u_{i}\right|^{2} \leqslant C r_{i}^{2 d-s} r_{i}^{2 \beta} n^{-2 \beta / d} \leqslant n^{-\frac{2 \beta}{d}+\frac{\alpha(2 d-s+2 \beta)}{d(\alpha+d)}}=n^{\frac{-2 \beta d+2 \alpha d-s \alpha}{d(\alpha+d)}} \leqslant 1,
$$

where, for the last inequality, we used (1.15). The energy of $h_{i}$ is estimated by scaling as in (7.13) to be

$$
\int_{B_{i} \times\left[-r_{i}, r_{i}\right]^{k}}|y|^{\gamma}\left|\left(\nabla h_{i}\right)_{\eta}\right|^{2} \leqslant c_{d, s} g(\eta)+ \begin{cases}C m_{i}^{s / d} & \text { in case (1.2) } \\ -\frac{c_{d, s}}{d} \log m_{i}+C & \text { in cases (1.3)-(1.4). }\end{cases}
$$

Combining these estimates and using (1.35)-(1.36), we find that in all cases

$$
\int_{\mathcal{C}_{i}}|y|^{\gamma}\left|\left(\nabla v_{i}\right)_{\eta}\right|^{2} \leqslant C_{\eta}+\frac{\min _{\mathcal{A}_{m_{i}}} \mathcal{W}}{m_{i}}=C_{\eta}+\left|\mathcal{C}_{i}\right| \min _{\mathcal{A}_{m_{i}}}
$$

since $\int_{\mathcal{C}_{i}} \mu_{V}^{\prime}=1$ and $m_{i}=f_{\mathcal{C}_{i}} \mu_{V}^{\prime}=\frac{1}{\left|\mathcal{C}_{i}\right|}$. 
The total contribution of the cells in $\Sigma_{\text {bound }}^{\prime}$ is bounded by the number of those cells (since there $m_{i}$ is bounded below), itself proportional to the volume of that strip i.e. $n^{\frac{d-1}{d}}$. The number of cells in $\Sigma^{\prime} \backslash \Sigma_{T}^{\prime}$ is on the other hand bounded by

$$
\begin{aligned}
\sum_{j=1}^{J} N_{j} & \leqslant C\left|\partial \Sigma^{\prime}\right| n^{-\alpha / d} \sum_{j=1}^{J}\left(t_{j}-t_{j-1}\right) t_{j}^{\alpha} \leqslant C\left|\partial \Sigma^{\prime}\right| n^{-\alpha / d} \int_{0}^{T} t^{\alpha} d t \\
& =C|\partial \Sigma| n^{\frac{d-1-\alpha}{d}} T^{1+\alpha} \leqslant C \underline{m}^{1+1 / \alpha} n,
\end{aligned}
$$

where we have used the properties (7.22) and inserted the upper bound $T \leqslant C \underline{m}^{1 / \alpha} n^{1 / d}$. As soon as $n$ is large enough, we may thus bound the total contribution of all the cells $\mathcal{C}_{i}$ as follows:

$$
\int|y|^{\gamma}\left|\Phi_{\eta}\left(E_{\text {bound }}\right)\right|^{2} \leqslant C_{\eta} n \underline{m}^{1+1 / \alpha}+\sum_{i}\left|\mathcal{C}_{i}\right| \min _{\mathcal{A}_{m_{i}}} \mathcal{W} .
$$

Finally, setting

$$
E=E_{\text {bound }}+E_{\text {int }} \quad \text { in } \mathbb{R}^{d+k}
$$

and letting

$$
\Lambda=\Lambda_{\text {int }} \cup \bigcup_{i}\left\{p_{i}\right\}
$$

then $\Lambda$ is made of $\int_{\Sigma^{\prime}} \mu_{V}^{\prime}=n$ points, which are all well separated, and $E$ satisfies

$$
-\operatorname{div}\left(|y|^{\gamma} E\right)=c_{d, s}\left(\sum_{p \in \Lambda} \delta_{p}-\mu_{V}^{\prime} \delta_{\mathbb{R}^{d}}\right) \quad \text { in } \mathbb{R}^{d+k} .
$$

Combining the estimates (7.14) and (7.29), we have

$$
\begin{aligned}
\int_{\mathbb{R}^{d+k}}|y|^{\gamma}\left|E_{\eta}\right|^{2} \leqslant & \sum_{K \in \mathcal{K}_{n}} m_{K}|K|\left(c_{d, s} g(\eta)\right. \\
& \left.+C_{\eta} o_{R}(1)\right)+\sum_{K \in \mathcal{K}_{n}}|K|\left(\min _{\mathcal{A}_{m_{K}}} \mathcal{W}+m_{K}^{1+s / d} \frac{\varepsilon}{4 \bar{m}^{s / d}}+C m_{K}^{1+\frac{s}{d}+\frac{1}{2}-\frac{s}{2 d}} \eta^{\frac{d-s}{2}}\right) \\
& +C_{\eta} n \underline{m}^{1+1 / \alpha}+\sum_{i}\left|\mathcal{C}_{i}\right| \min _{\mathcal{A}_{m_{i}}} \mathcal{W}+C_{\eta} n \underline{m}^{1+1 / \alpha}
\end{aligned}
$$

First, we note that

$$
\sum_{K \in \mathcal{K}_{n}} m_{K}|K|=\int_{\Sigma_{\text {int }}^{\prime}} \mu_{V}^{\prime} \leqslant n
$$

Second, in view of the continuity of $\mu_{V}$ and that of $m \mapsto \min _{\mathcal{A}_{m}} \mathcal{W}$ (see (1.35)-(1.36)), we may recognize a Riemann sum and write (using the fact that $m_{K} \leqslant \bar{m}$ by (1.14))

$$
\begin{aligned}
\int_{\mathbb{R}^{d+k}}|y|^{\gamma}\left|E_{\eta}\right|^{2} \leqslant & n c_{d, s} g(\eta)+\int_{\Sigma^{\prime}} \min _{\mathcal{A}_{\mu^{\prime}(x)}} \mathcal{W} d x+\frac{n \varepsilon}{4} \\
& +C_{\eta} n \underline{m}^{1+1 / \alpha}+C_{\eta} o_{R}(n)+C \bar{m}^{\frac{1}{2}+\frac{s}{2 d}} \eta^{\frac{d-s}{2}}
\end{aligned}
$$




$$
\begin{aligned}
\leqslant & n c_{d, s} g(\eta)+n\left(\int_{\Sigma} \min _{\mathcal{A}_{\mu_{V}(x)}} \mathcal{W} d x+\frac{\varepsilon}{4}\right)+C_{\eta} n \underline{m}^{1+1 / \alpha}+C_{\eta} o_{R}(n) \\
& +C \bar{m}^{\frac{1}{2}+\frac{s}{2 d}} \eta^{\frac{d-s}{2}} .
\end{aligned}
$$

Step 4. Conclusion.

We may now define our test configuration as

$$
\mathbf{x}=\left\{x_{i}=n^{-1 / d} x_{i}^{\prime}\right\}_{i=1}^{n} \text { where } \Lambda=\left(x_{1}^{\prime}, \ldots, x_{n}^{\prime}\right),
$$

and let $h_{n}^{\prime}$ be the associated potential as in (1.44). Note that the configuration depends on $R$ and $\eta$, and that the points $x_{i}^{\prime}$ are separated by distances independent of $R, \eta$, and $n$. For $\eta$ small enough, we are thus in the equality case in Lemma 2.3, and using the splitting formula (1.49), we deduce that

$$
\begin{aligned}
& \limsup _{n \rightarrow \infty} n^{-1-s / d}\left(H_{n}\left(x_{1}, \ldots, x_{n}\right)-n^{2} \mathcal{E}\left(\mu_{V}\right)\right) \\
& \quad \leqslant \limsup _{n \rightarrow \infty}\left(\frac{1}{n c_{d, s}} \int_{\mathbb{R}^{d+k}}|y|^{\gamma}\left|\nabla h_{n, \eta}^{\prime}\right|^{2}-g(\eta)\right)+C \eta^{\frac{d-s}{2}},
\end{aligned}
$$

in case (1.2), respectively

$$
\begin{aligned}
& \limsup _{n \rightarrow \infty} n^{-1}\left(H_{n}\left(x_{1}, \ldots, x_{n}\right)-n^{2} \mathcal{E}\left(\mu_{V}\right)+\frac{n}{d} \log n\right) \\
& \quad \leqslant \limsup _{n \rightarrow \infty}\left(\frac{1}{n c_{d, s}} \int_{\mathbb{R}^{d+k}}|y|^{\gamma}\left|\nabla h_{n, \eta}^{\prime}\right|^{2}-g(\eta)\right)+C \eta^{\frac{d}{2}},
\end{aligned}
$$

in the cases (1.3)-(1.4), where we used that all the points are in $\Sigma$ where the function $\zeta$ vanishes. We now note that projecting $E$ onto gradients decreases the energy:

$$
\begin{aligned}
\int_{\mathbb{R}^{d+k}}|y|^{\gamma}\left|E_{\eta}\right|^{2}= & \int_{\mathbb{R}^{d+k}}|y|^{\gamma}\left|\nabla h_{n, \eta}^{\prime}\right|^{2}+\int_{\mathbb{R}^{d+k}}|y|^{\gamma}\left|E_{\eta}-\nabla h_{n, \eta}^{\prime}\right|^{2} \\
& +2 \int_{\mathbb{R}^{d+k}}|y|^{\gamma} \nabla h_{n, \eta}^{\prime} \cdot\left(E_{\eta}-\nabla h_{n, \eta}^{\prime}\right) \\
\geqslant & \int_{\mathbb{R}^{d+k}}|y|^{\gamma}\left|\nabla h_{n, \eta}^{\prime}\right|^{2}-2 \int_{\mathbb{R}^{d+k}} h_{n, \eta}^{\prime} \operatorname{div}\left(|y|^{\gamma}\left(E_{\eta}-\nabla h_{n, \eta}^{\prime}\right)\right) \\
= & \int_{\mathbb{R}^{d+k}}|y|^{\gamma}\left|\nabla h_{n, \eta}^{\prime}\right|^{2}
\end{aligned}
$$

where we have used that $-\operatorname{div}\left(|y|^{\gamma}\left(E_{\eta}-\nabla h_{n, \eta}^{\prime}\right)\right)=0$ by the definition of $h_{n, \eta}^{\prime}$ and (7.30). Combining (7.33)-(7.36), the proof is concluded by taking successively $n \rightarrow \infty$, then $R$ large enough, $\underline{m}$ small enough, and $\eta$ small enough (and changing the configuration of points accordingly) to conclude that we can get

$$
\limsup _{n \rightarrow \infty} n^{-1-s / d}\left(H_{n}\left(x_{1}, \ldots, x_{n}\right)-n^{2} \mathcal{E}\left(\mu_{V}\right)\right) \leqslant\left(\frac{1}{c_{d, s}} \int_{\Sigma} \min _{\mathcal{A}_{\mu_{V}(x)}} \mathcal{W} d x+\frac{\varepsilon}{2}\right),
$$

and the analog in cases (1.3)-(1.4). Finally, inserting (1.35)-(1.36) and the definition of $\widetilde{\mathcal{W}}$, we obtain the upper bound result, together with the fact that indeed 
$\min \tilde{\mathcal{W}}=\frac{1}{c_{d, s}} \int_{\Sigma} \min _{\mathcal{A}_{\mu_{V}(x)}} \mathcal{W}$ (by comparing the upper bound with the lower bound result, Proposition 5.2). Since the points are well separated, we may also move each of them by a distance $r$ small enough and keep the same estimate up to an additional error $\varepsilon / 2$, in a neighborhood $A_{n}$ of the configuration. The details are identical to those in [44].

This concludes the proof of Proposition 7.3.

Theorem 4 then follows by comparison with Proposition 5.2. Theorem 6 follows from all the previous results exactly as in [44]. The details are left to the reader.

\section{Additional remarks and acknowledgments}

After this work was completed, we learned of a related forthcoming work by Hardin et al. [31], who obtain the existence of the same order asymptotic expansion of the minimal energy for the Riesz or logarithmic interaction on a flat torus for any $s<d$.

This paper benefited from comments and discussions with Thomas Leblé, whom we would like to warmly thank here.

The first author was supported by a postdoctoral fellowship of the Fondation Sciences Mathématiques de Paris. The second author was supported by a EURYI award.

\section{References}

1. G. Alberti and S. MÜller, A new approach to variational problems with multiple scales, Comm. Pure Appl. Math. 54(7) (2001), 761-825.

2. A. Avila, Y. Last And B. Simon, Bulk universality and clock spacing of zeros for ergodic Jacobi matrices with absolutely continuous spectrum, Anal. PDE 3(1) (2010), 81-108.

3. P. T. Bateman and E. Grosswald, On Epstein's zeta function, Acta Arith. 9(4) (1964), 365-373.

4. M. E. BECKER, Multiparameter groups of measure-preserving transformations: a simple proof of Wiener's ergodic theorem, Ann. Prob. 9(3) (1981), 504-509.

5. L. BÉtermin, Renormalized energy and asymptotic expansion of optimal logarithmic energy on the sphere, arXiv:1404.4485.

6. F. Bethuel, H. Brezis And F. HÉlein, Ginzburg-Landau Vortices, Progress in Nonlinear Partial Differential Equations and Their Applications (Birkhäuser, 1994).

7. S. Borodachov, D. H. Hardin and E. B. SafF, Minimal discrete energy on the sphere and other manifolds (forthcoming).

8. A. Borodin AND S. SERFATY, Renormalized energy concentration in random matrices, Comm. Math. Phys. 320(1) (2013), 199-244.

9. H. J. Brascamp And E. H. Lieb, Some inequalities for Gaussian measures and the long-range order of the one-dimensional plasma, in Functional Integration and its Applications(ed. A. M. Arthurs), (Clarendon Press, 1975).

10. J. S. Brauchart, P. D. Dragnev and E. B. Saff, Riesz extremal measures on the sphere for axis-supported external fields, J. Math. Anal. Appl. 356(2) (2009), 769-792.

11. J. S. Brauchart, P. D. Dragnev and E. B. Saff, Riesz external field problems on the hypersphere and optimal point separation, Potential Anal. (to appear).

12. J. S. Brauchart, D. P. Hardin and E. B. SafF, The next order term for optimal Riesz and logarithmic energy asymptotics on the sphere, in Recent Advances in Orthogonal 
Polynomials, Special Functions, and their Applications, Contemporary Mathematics, Volume 578, pp. 31-61 (American Mathematical Society, Providence, RI, 2012).

13. L. A. Caffarelli and L. Sillvestre, An extension problem related to the fractional Laplacian, Comm. Partial Differential Equations 32(7-9) (2007), 1245-1260.

14. L. A. Caffarelli, S. Salsa and L. Silvestre, Regularity estimates for the solution and the free boundary of the obstacle problem of the fractional Laplacian, Invent. Math. 171(2) (2008), 425-461.

15. L. A. Caffarelli, J.-M. Roquejoffre And Y. Sire, Variational problems for free boundaries for the fractional Laplacian, J. Eur. Math. Soc. (JEMS) 12(5) (2010), $1151-1179$.

16. J. W. S. Cassels, On a problem of Rankin about the Epstein zeta-function, Proc. Glasg. Math. Assoc. 4 (1959), 73-80.

17. T. H. Chan, Finding almost squares, Acta Arith. 121 (2006), 221-232.

18. J. Chatard, Applications des propriétés de moyenne d'un groupe localement compact à la théorie ergodique, Ann. I. H. P. (B) Probab. Stat. 6(4) (1970), 307-326.

19. D. Chafaï, N. Gozlan and P.-A. Zitt, First order global asymptotics for confined particles with singular pair repulsion, Ann. Appl. Probab. 24(6) (2014), 2371-2413.

20. G. Choquet, Diamètre transfini et comparaison de diverses capacités, Technical report, Faculté des Sciences de Paris (1958).

21. S. Chowla And A. Selberg, On Epstein's zeta-function, J. Reine Angew. Math. 227 (1967), 86-110.

22. H. Cohn and N. Elkies, New upper bounds on sphere packings. I, Ann. of Math. (2) 157(2) (2004), 689-714.

23. H. Cohn And A. Kumar, Universally optimal distribution of points on spheres, J. Amer. Math. Soc. 20(1) (2007), 99-148 (electronic).

24. P. H. Diananda, Notes on two lemmas concerning the Epstein zeta-function, Proc. Glasg. Math. Assoc. 6 (1964), 202-204.

25. V. Ennola, A lemma about the Epstein zeta-function, Proc. Glasg. Math. Assoc. 6 (1964), $198-201$.

26. V. Ennola, On a problem about the Epstein zeta function, Proc. Cambridge Philos. Soc. 60 (1964), 855-875.

27. E. B. Fabes, C. E. Kenig and R. P. Serapioni, The local regularity of solutions of degenerate elliptic equations, Comm. Partial Differential Equations 7(1) (1982), 77-116.

28. L. Flatley and F. Theil, Face-centered cubic crystallization of atomistic configurations, Arch. Rational. Mech. Anal. (2015), doi:10.1007/s00205-015-0862-1.

29. P. J. ForRester, London Mathematical Society Monographs Series, Volume 34 (Princeton University Press, 2010).

30. O. Frostman, Potentiel d'équilibre et capacité des ensembles avec quelques applications à la théorie des fonctions, Meddelanden Mat. Sem. Univ. Lund $3115 \mathrm{~s}$ (1935).

31. D. H. Hardin, E. B. Saff, B. Z. Simanek and Y. Su, Second order asymptotics for long-range Riesz potentials on flat torii (in preparation).

32. L. Hörmander, The Analysis of Linear Partial Differential Operators, I: Distribution Theory and Fourier Analysis (Springer, 1983).

33. N. S. LandKof, Foundations of Modern Potential Theory (Springer, 1972).

34. T. LeBlé, A uniqueness result for minimizers of the 1D log-gas renormalized energy, J. Funct. Anal. (to appear), arXiv:1408.2283.

35. A. LENARD, Exact statistical mechanics of a one-dimensional system with Coulomb forces, J. Math. Phys. 2 (1961), 682-693.

36. A. LenARD, Exact statistical mechanics of a one-dimensional system with Coulomb forces III: statistics of the electric field, J. Math. Phys. 4 (1963), 533-543. 
37. E. LIEB, Personal communication.

38. S. A. Molchanov And E. Ostrovski, Symmetric stable processes as traces of degenerate diffusion processes, Theory Probab. Appl. 14 (1969), 128-131.

39. H. L. Montgomery, Minimal theta functions, Glasg. Math. J. 30(1) (1988), 75-85.

40. A. P. Prudnikov, Y. A. Brychkov and O. I. Marichev, Integrals and Series, Vol. 1: Elementary Functions (Gordon and Breach, 1986).

41. C. Radin, The ground state for soft disks, J. Stat. Phys. 26 (1981), 365-373.

42. R. A. Rankin, A minimum problem for the Epstein zeta function, Proc. Glasg. Math. Assoc. 1 (1953), 149-158.

43. S. Rota Nodari And S. Serfaty, Renormalized energy equidistribution and local charge balance in 2D Coulomb systems, Int. Math. Res. Not. IMRN (2014), doi:10.1093/imm/r nu031.

44. N. Rougerie And S. Serfaty, Higher dimensional Coulomb gases and renormalized energy functionals, Comm. Pure Appl. Math. (to appear), doi:10.1002/cpa.21570.

45. E. SafF And A. KuiJlaArs, Distributing many points on a sphere, Math. Intelligencer 19(1) (1997), 5-11.

46. E. B. Saff And V. Totik, Logarithmic Potentials with External Fields, Grundlehren der mathematischen Wissenchaften, vol. 316 (Springer, Berlin, 1997).

47. E. Sandier and S. Serfaty, From the Ginzburg-Landau model to vortex lattice problems, Comm. Math. Phys. 313 (2012), 635-743.

48. E. Sandier and S. Serfaty, 2D Coulomb gases and the renormalized energy, Ann. Probab. (to appear).

49. E. Sandier And S. Serfaty, 1D log gases and the renormalized energy: crystallization at vanishing temperature, Prob. Theor. Rel. Fields (2013), (to appear), arXiv:1303.2968, doi:10.1007/s00440-014-0585-5.

50. P. Sarnak and A. Strömbergsson, Minima of Epstein's zeta function and heights of flat tori, Inv. Math. 165(1) (2006), 115-151.

51. S. Serfaty, Coulomb Gases and Ginzburg-Landau Vortices, Zurich Lectures in Advanced Mathematics, (European Mathematical Society, 2015).

52. L. Silvestre, The regularity of the obstacle problem for a fractional power of the Laplace operator, Comm. Pure Appl. Math. 60(1) (2007), 67-112. 